\title{
Sampling discretization and related problems
}

\author{
B. Kashin*, E. Kosov† I. Limonova $\ddagger$ and V. Temlyakov ${ }^{\S}$
}

\begin{abstract}
This survey addresses sampling discretization and its connections with other areas of mathematics. The survey concentrates on sampling discretization of norms of elements of finite-dimensional subspaces. We present here known results on sampling discretization of both integral norms and the uniform norm beginning with classical results and ending with very recent achievements. We also show how sampling discretization connects to spectral properties and operator norms of submatrices, embedding of finite-dimensional subspaces, moments of marginals of high-dimensional distributions, and learning theory. Along with the corresponding results, important techniques for proving those results are discussed as well.
\end{abstract}

Keywords and phrases: Sampling discretization, operator norms of submatrices, optimal embedding of finite-dimensional subspaces, random matrices, learning theory.

*Steklov Institute of Mathematics, Moscow Center for Fundamental and Applied Mathematics,

${ }^{\dagger}$ Lomonosov Moscow State University, National Research University Higher School of Economics,

${ }^{\ddagger}$ Lomonosov Moscow State University, Moscow Center for Fundamental and Applied Mathematics,

$\S$ University of South Carolina, Steklov Institute of Mathematics, Lomonosov Moscow State University, and Moscow Center for Fundamental and Applied Mathematics. 


\section{Contents}

1 Introduction $\quad 2$

2 Discretization $\quad 19$

2.1 Trigonometric polynomials . . . . . . . . . . . . . . . 21

2.2 General subspaces . . . . . . . . . . . . . . . 26

2.3 Real algebraic polynomials . . . . . . . . . . . . . . 33

2.4 Complex algebraic polynomials . . . . . . . . . . . . 37

2.5 Sampling recovery . . . . . . . . . . . . . . . . . 39

2.6 Some comments on techniques . . . . . . . . . . . . . . 44

2.7 Some further comments and open problems . . . . . . . . . . . 49

3 Spectral properties and operator norms of submatrices $\quad 51$

3.1 Euclidean case . . . . . . . . . . . . . 53

$3.2 p=2, q=1 \ldots \ldots \ldots \ldots 6$

3.3 General case . . . . . . . . . . . . . . . . 57

3.4 Algorithms . . . . . . . . . . . . . . . . . 59

4 Connections with other problems $\quad \mathbf{6 0}$

4.1 Moments of marginals of high-dimensional distributions . . . . 60

4.2 Embedding of finite-dimensional subspaces of $L_{q}$ into $\ell_{q}^{m}$. . 67

4.3 Sparse approximation . . . . . . . . . . . . . . . 71

4.4 Supervised learning theory . . . . . . . . . . . . . . . 74

\section{Introduction}

Discretization is an essential step in making a continuous problem computationally feasible. The construction of good sets of points in a multidimensional domain is a fundamental problem of pure and computational mathematics.

A classical example of a discretization problem is to estimate metric entropy (covering numbers, entropy numbers). Bounds for the $\varepsilon$-entropy of function classes are inherently important and also important in connection with other fundamental problems. Numerical integration is another prominent example of a discretization problem. We now briefly illustrate difficulties in finding good points for covering and for numerical integration on the example of discretizing the $d$-dimensional unit cube $[0,1]^{d}$. There are different 
ways of doing that. For example, we can interpret $[0,1]^{d}$ as a compact set of $\mathbb{R}^{d}$ and use the idea of covering numbers (metric entropy). With this approach, for instance in the case of $\ell_{\infty}$ norm, we can find optimal coverings. For a given $n \in \mathbb{N}$ the regular grid with coordinates at the centers of intervals $[(k-1) / n, k / n], k=1, \ldots, n$, provides a net for an optimal $\ell_{\infty}$ covering with the number of points $N=n^{d}$. Very often the unit cube $[0,1]^{d}$ plays the role of a domain, where smooth functions of $d$ variables are defined and we are interested in discretizing some continuous operations with these functions. Numerical integration is such an example. It turns out that the regular grids mentioned above are very far from being a good discretization of $[0,1]^{d}$ for numerical integration of functions with mixed smoothness.

A problem of optimal recovery is one more example of a discretization problem. This problem turns out to be very difficult for the mixed smoothness classes of multivariate functions, which are important in applications. It is not solved even in the case of the $L_{2}$ norm.

A standard approach to solving a continuous problem numerically - the Galerkin method - suggests searching for an approximate solution from a given finite-dimensional subspace. A standard way to measure an error of approximation is an appropriate $L_{q}$ norm, $1 \leq q \leq \infty$. Thus, the problem of discretization of the $L_{q}$ norms of functions from a given finite-dimensional subspace arises in a very natural way. The first results in this direction were obtained in the 1930s by Bernstein, by Marcinkiewicz and by MarcinkiewiczZygmund for discretization of the $L_{q}$ norms of the univariate trigonometric polynomials. Even though this problem is very important in applications, its systematic study has begun only recently.

The problem of arranging points in a multidimensional domain, in particular the problem of optimal spherical codes, is also a fundamental problem in coding theory. Finding optimal spherical codes is equivalent to building large incoherent dictionaries in $\mathbb{R}^{d}$ in compressed sensing. Compressed sensing is an actively developing area of numerical analysis, which is very important in processing large data sets, in particular in signal and image processing (see, for instance, [43] and [153], Ch. 5).

The goals of this paper are to survey the results, and connect together ideas, methods, and results from different areas of research related to problems of discretization and recovery in the case of finite-dimensional subspaces. We note that this survey does not cover some classical areas where discretization problems are studied. For instance, we do not discuss discretization in spaces of analytic functions. The reader can find such results in [135] and 
[116]. Many results on discretization discussed in this survey are obtained with the aid of probabilistic technique. The authors of those results use a variant of the concentration of measure phenomenon, the chaining technique, and bounds of the entropy numbers. We note that the fundamental idea of chaining goes back to the 1930s, when it was suggested by A.N. Kolmogorov in 1934 for finding sufficient conditions for almost everywhere continuity of trajectories of random processes. The first publication of his proof (with his approval) appeared in the paper by E. Slutsky [139] (see also [140]). Similar probabilistic techniques were used in other areas of research: Spectral properties and operator norms of submatrices, embedding of finite-dimensional subspaces into $\ell_{q}^{m}$, moments of marginals of high-dimensional distributions, and learning theory.

We now proceed to precise settings and to a more detailed discussion.

Discretization. Let $\Omega$ be a compact subset of $\mathbb{R}^{d}$ with the probability measure $\mu$. By sampling discretization in a broad sense we understand replacement of the measure $\mu$ by a discrete measure $\mu_{m}$ supported on a set of $m$ points from $\Omega$, which keeps some properties of $\mu$. We would like a discrete replacement $\mu_{m}$ to represent (approximate) the original measure $\mu$ well. A specific (narrow) sampling discretization setting is determined by a criterion on the error of approximation of $\mu$ by $\mu_{m}$. There are classical ways to measure how close two measures are, for example, the Hellinger distance and the Kullback-Leibler information (see, for instance, [91] and the books [123] and [153], Section 4.2.3), which are important in probability theory and statistics. Our criteria of good approximation of $\mu$ by $\mu_{m}$ are motivated by applications in approximation theory and numerical analysis. We begin with the classical problem of numerical integration, which provides an example of such criterion. Numerical integration seeks good ways of approximating an integral

$$
\int_{\Omega} f(\mathbf{x}) d \mu(\mathbf{x})
$$

by an expression of the form

$$
\Lambda_{m}(f, \xi):=\sum_{j=1}^{m} \lambda_{j} f\left(\xi^{j}\right), \quad \xi=\left\{\xi^{j}\right\}_{j=1}^{m} \subset \Omega, \quad j=1, \ldots, m .
$$

It is clear that we must assume that $f$ is integrable and defined at the points $\xi^{1}, \ldots, \xi^{m}$. Expression (1.1) is called a cubature formula $(\xi, \Lambda)$ (if $\Omega \subset \mathbb{R}^{d}$, $d \geq 2$ ) or a quadrature formula $(\xi, \Lambda)$ (if $\Omega \subset \mathbb{R}$ ) with nodes $\xi=\left\{\xi^{j}\right\}_{j=1}^{m}$ and 
weights $\Lambda:=\left\{\lambda_{j}\right\}_{j=1}^{m} \subset \mathbb{R}$ or $\subset \mathbb{C}$. We do not impose any a priori restrictions on nodes and weights. Some nodes may coincide and both positive and negative weights are allowed. The above setting means that in the integration problem we replace the measure $\mu$ by the discrete measure (signed measure) $\mu_{m}$ such that $\mu_{m}\left(\xi^{j}\right)=\lambda_{j}, j=1, \ldots, m$.

Some classes of cubature formulas are of special interest. For instance, cubature formulas, which have equal weights $1 / m$, are important in applications. We use a special notation for these cubature formulas

$$
Q_{m}(f, \xi):=\frac{1}{m} \sum_{j=1}^{m} f\left(\xi^{j}\right) .
$$

In this notation $Q$ stands for Quasi-Monte Carlo - a standard in some areas of research name for the above cubature formulas.

Typically, one is interested in good cubature formulas for a given function class. The term good can be understood in different ways. Cubature formulas providing exact numerical integration for functions from a given class can be considered best. If a cubature formula is not exact on a given class, then we need to introduce a concept of error. Following the standard approach, for a function class $\mathbf{W}$ we introduce the errors of the cubature formulas $\Lambda_{m}(\cdot, \xi)$ and $Q_{m}(\cdot, \xi)$ by

$$
\begin{aligned}
& \Lambda_{m}(\mathbf{W}, \xi):=\sup _{f \in \mathbf{W}}\left|\int_{\Omega} f d \mu-\Lambda_{m}(f, \xi)\right|, \\
& Q_{m}(\mathbf{W}, \xi):=\sup _{f \in \mathbf{W}}\left|\int_{\Omega} f d \mu-Q_{m}(f, \xi)\right| .
\end{aligned}
$$

The quantities $\Lambda_{m}(\mathbf{W}, \xi)$ and $Q_{m}(\mathbf{W}, \xi)$ are classical characteristics of the quality of given cubature formulas. This setting is called the worst case setting in the Information Based Complexity (see, e.g., [174] and [164]). Notice that the above characteristics provide an absolute error independent of an individual function from the class. A typical class $\mathbf{W}$ in the numerical integration problem is a smoothness class of functions continuous on $\Omega$, for instance, it might be the unit ball of a Sobolev, Nikol'skii, or Besov space. Thus, for a given class $\mathbf{W}$ the error $\Lambda_{m}(\mathbf{W}, \xi)$ shows how close measures $\mu$ and $\mu_{m}$ are, where $\mu_{m}$ is the discrete measure, concentrated on the set $\xi=\left\{\xi^{j}\right\}_{j=1}^{m}$ with weights $\Lambda:=\left\{\lambda_{j}\right\}_{j=1}^{m}$, i.e. $\mu_{m}\left(\xi^{j}\right)=\lambda_{j}, j=1, \ldots, m$. In 
particular, if $\mathbf{W}$ is a unit ball of a Banach space embedded in $\mathcal{C}(\Omega)$, then $\Lambda_{m}(\mathbf{W}, \xi)$ is the norm of the difference $\mu-\mu_{m}$ in the dual norm.

Recently, in a number of papers (see [154], [155], [156], [33], [158], [34], [35], [86]) a systematic study of a variant of the numerical integration problem has begun. It is the problem of discretization of the $L_{q}$ norms of elements from a given function class $\mathbf{F}$. By $L_{q}, 1 \leq q<\infty$, norm we understand

$$
\|f\|_{q}:=\|f\|_{L_{q}(\Omega, \mu)}:=\left(\int_{\Omega}|f|^{q} d \mu\right)^{1 / q} .
$$

By $L_{\infty}$ norm we understand the uniform norm of continuous functions

$$
\|f\|_{\infty}:=\max _{\mathbf{x} \in \Omega}|f(\mathbf{x})|
$$

and with some abuse of notation we occasionally write $L_{\infty}(\Omega)$ for the space $\mathcal{C}(\Omega)$ of continuous functions on $\Omega$.

By discretization of the $L_{q}$ norm we understand a replacement of the measure $\mu$ by a discrete measure $\mu_{m}$ with support on a set $\xi=\left\{\xi^{j}\right\}_{j=1}^{m} \subset \Omega$ in such a way that the error $\left|\|f\|_{L_{q}(\Omega, \mu)}^{q}-\|f\|_{L_{q}\left(\Omega, \mu_{m}\right)}^{q}\right|$ is small for $f \in \mathbf{F}$. This means that integration with respect to $\mu$ is replaced by an appropriate cubature formula. Thus, integration is replaced by evaluation of a function $f$ at a finite set $\xi$ of points. This is why this way of discretization is called sampling discretization. The reader can find a corresponding discussion in a recent survey [33]. The first results in sampling discretization were obtained by Bernstein [9] and [10] (see also [176], Ch.10, Theorem (7.28)) for discretization of the uniform norm of the univariate trigonometric polynomials in 1931-1932. In this case $\mathbf{F}$ is the unit $L_{\infty}$-ball of a subspace of the univariate trigonometric polynomials of a given degree. The first results in sampling discretization of the integral norms $L_{q}, 1 \leq q<\infty$, were obtained by Marcinkiewicz $(1<q<\infty)$ and by Marcinkiewicz-Zygmund $(1 \leq q \leq \infty)$ (see [176], Ch.10, Theorems (7.5) and (7.28)) for discretization of the $L_{q}$ norms of the univariate trigonometric polynomials in 1937. Therefore, we also call sampling discretization results Bernstein-type theorems in the case of discretization of the uniform norm of functions from a finite-dimensional subspace and Marcinkiewicz-type theorems in the case of integral norms of functions from a finite-dimensional subspace (see [154], [155], [33]). In the literature this kind of results is also known under the name MarcinkiewiczZygmund inequalities (see, for instance, [36], [50] and references therein). 
Recently, a substantial progress in sampling discretization has been made in [154], [155], [78], [33], [34], [35], [86], and [96].

It will be convenient for us to use the following notation. For given sets $\xi:=\left\{\xi^{j}\right\}_{j=1}^{m}$ of sampling points and $\Lambda:=\left\{\lambda_{j}\right\}_{j=1}^{m} \subset \mathbb{R}$ of weights, and for a class $\mathbf{F} \subset L_{q}(\Omega, \mu), 1 \leq q<\infty$, of continuous on $\Omega$ functions we define

$$
\operatorname{er}\left(\mathbf{F}, \xi, L_{q}\right):=\left.\sup _{f \in \mathbf{F}}\left|\|f\|_{q}^{q}-\frac{1}{m} \sum_{j=1}^{m}\right| f\left(\xi^{j}\right)\right|^{q} \mid
$$

and

$$
\operatorname{er}\left(\mathbf{F}, \xi, \Lambda, L_{q}\right):=\left.\sup _{f \in \mathbf{F}}\left|\|f\|_{q}^{q}-\sum_{j=1}^{m} \lambda_{j}\right| f\left(\xi^{j}\right)\right|^{q} \mid .
$$

It is clear that the above errors of discretization on the class $\mathbf{F}$ coincide with the numerical integration errors on the class $\mathbf{W}=\mathbf{F}^{q}:=\left\{|f|^{q}: f \in \mathbf{F}\right\}$

$$
\operatorname{er}\left(\mathbf{F}, \xi, L_{q}\right)=Q_{m}\left(\mathbf{F}^{q}, \xi\right), \quad \operatorname{er}\left(\mathbf{F}, \xi, \Lambda, L_{q}\right)=\Lambda_{m}\left(\mathbf{F}^{q}, \xi\right) .
$$

A special case, when $\mathbf{F}$ is a unit ball of a given $N$-dimensional subspace $X_{N}$ of $L_{q}(\Omega)$, plays a very important role in sampling discretization. Denote $X_{N}^{q}:=\left\{f: f \in X_{N},\|f\|_{q} \leq 1\right\}$. If $\operatorname{er}\left(\mathbf{F}, \xi, L_{q}\right)=\varepsilon$, then it is guaranteed that for any $f \in \mathbf{F}$ we have the following error bound, which does not depend on $f$ and only depends on the function class $\mathbf{F}$

$$
\left.\left|\|f\|_{q}^{q}-\frac{1}{m} \sum_{j=1}^{m}\right| f\left(\xi^{j}\right)\right|^{q} \mid \leq \varepsilon
$$

In the case $\mathbf{F}=X_{N}^{q}, \varepsilon<1$, we can write (1.2) for $f$ such that $\|f\|_{q}=1$ and obtain the following relative error bounds

$$
(1-\varepsilon)\|f\|_{q}^{q} \leq \frac{1}{m} \sum_{j=1}^{m}\left|f\left(\xi^{j}\right)\right|^{q} \leq(1+\varepsilon)\|f\|_{q}^{q} \quad \text { for all } \quad f \in X_{N} .
$$

Inequalities of the form (1.3) are known under the name of the MarcinkiewiczZygmund inequalities. We point out that inequalities (1.3) consist of two inequalities - the left inequality, which provides the lower bound for the discrete norm, and the right inequality, which provides the upper bound for the discrete norm. These one-sided inequalities are also of interest and they are discussed here as well. 
In this survey we concentrate on results on sampling discretization of the integral norms of elements of finite-dimensional subspaces. There are some known results on the behavior of the quantities $\operatorname{er}\left(\mathbf{F}, \xi, L_{q}\right)$ and $\operatorname{er}\left(\mathbf{F}, \xi, \Lambda, L_{q}\right)$ for $\mathbf{F}$ being an infinitely dimensional function class (see, for instance, [158]). We do not discuss these results here. We stress that in the above setting we discretize the $\|f\|_{q}^{q}$. There are results on optimal estimation of the $\|f\|$ under assumption that $f \in \mathbf{F}$ (see [171]). At a first glance the problems of estimation of $\|f\|$ and, say, estimation of $\|f\|^{2}$, are very close. A simple inequality $\left|a^{2}-b^{2}\right| \leq 2 M|a-b|$ for numbers satisfying $|a| \leq M$ and $|b| \leq M$ shows that normally we can get an upper bound for estimation of $\|f\|^{2}$ in terms of the error of estimation of $\|f\|$. However, it turns out that the problems of optimal errors in sampling discretization of $\|f\|$ and $\|f\|^{2}$ are different as it is explained in [158].

The main goal of the sampling discretization theory is to find good sampling points for a given function class F. Naturally, this goal includes two steps. The first step consists of finding good bounds for the following optimal quantities

$$
\begin{aligned}
e r_{m}\left(\mathbf{F}, L_{q}\right) & :=\inf _{\xi^{1}, \ldots, \xi^{m}} \operatorname{er}\left(\mathbf{F}, \xi, L_{q}\right), \\
e r_{m}^{o}\left(\mathbf{F}, L_{q}\right):= & \inf _{\substack{\xi^{1}, \ldots, \xi_{m}^{m} \\
\lambda_{1}, \ldots, \lambda_{m}}} \operatorname{er}\left(\mathbf{F}, \xi, \Lambda, L_{q}\right) .
\end{aligned}
$$

We point out that the superscript $o$ in $\operatorname{er}_{m}^{o}\left(\mathbf{F}, L_{q}\right)$ stands for optimal over both the point sets and the weights. This notation is similar to the corresponding notations in numerical integration and discrepancy theory (see [157], p. 316). The second step consists of finding sampling points, which provide discretization errors close to the optimal ones. The tradeoff between accuracy and complexity is also an interesting question. In this survey we mostly present results on the first step.

In the above setting we are interested in the behavior of the error characteristics, which are optimal either over the point sets $\left(e r_{m}\right)$ or over both the point sets and the weights $\left(e r_{m}^{o}\right)$. The setting, when the point set $\xi:=\left\{\xi^{1}, \ldots, \xi^{m}\right\}$ is given (deterministically or randomly) and we want either to control the error $\operatorname{er}\left(\mathbf{F}, \xi, L_{q}\right)$ or to prove inequalities (1.3) is also interesting and important in applications (see, for instance, [4], [49], [118], [124], [141], and references therein) but not considered in the survey. This setting falls into sampling theory and the corresponding Marcinkiewicz-Zygmund inequalities are known under the name sampling theorem. 
In Section 2 we discuss results on sampling discretization of the $L_{q}, 1 \leq$ $q \leq \infty$, norm of elements of $N$-dimensional subspaces $X_{N}$ of the space $\mathcal{C}(\Omega)$ of functions continuous on $\Omega$. We call problems of that type the Marcinkiewicz discretization problems. These problems concern the upper bounds on the errors $e r_{m}\left(\mathbf{F}, L_{q}\right)$ and $e r_{m}^{o}\left(\mathbf{F}, L_{q}\right)$ where $\mathbf{F}=X_{N}^{q}$ is the $L_{q}$ unit ball of the subspace $X_{N}$.

In Section 2 we present two types of results on sampling discretization. Some results involve sampling discretization for specific $N$-dimensional subspaces, for instance, the trigonometric polynomials with frequencies from parallelepipeds or hyperbolic crosses, algebraic polynomials. In this case techniques from harmonic analysis, approximation theory, and number theory are used. Other results involve sampling discretization in general $N$ dimensional subspaces, which may satisfy some natural conditions such as the Nikol'skii-type inequality or inequalities on the entropy numbers of the $L_{q}$ unit ball of the $X_{N}$ in the uniform norm. In this case techniques from functional analysis and probability theory are used.

Submatrices. Section 3 is devoted to results on spectral properties, operator norms of submatrices, and related quantities of a given matrix, which are directly connected to the sampling discretization in finite-dimensional subspaces. The point is that the problem of discretizing functional systems can usually be reduced to the corresponding matrix problem. For many problems a given subspace can be replaced by the corresponding subspace consisting of piecewise constant functions with general metric properties to be preserved. An advantage of the matrix setting is that its analysis allows us to apply the iteration technique (see Section 3 and Subsection 4.2 for a detailed discussion).

Let us consider sampling discretization of the $L_{q}$ norm in the space $X_{N}=\operatorname{span}\left\{\psi_{1}, \ldots, \psi_{N}\right\}$ (see (1.3) above and (2.1) below). We want to find a set of $m$ points $\xi=\left\{\xi^{j}\right\}_{j=1}^{m}$ such that

$$
C_{1}\|f\|_{q}^{q} \leq \frac{1}{m} \sum_{j=1}^{m}\left|f\left(\xi^{j}\right)\right|^{q} \leq C_{2}\|f\|_{q}^{q}, \quad \forall f \in X_{N} .
$$

Then for $f=\sum_{i=1}^{N} b_{i} \psi_{i}$ and for the matrix $S:=\left[\psi_{i}\left(\xi^{j}\right)\right], j=1, \ldots, m$, $i=1, \ldots, N$, with $m$ rows (enumerated by $j$ ) and $N$ columns (enumerated by $i$ ) inequalities (1.4) mean that

$$
C_{1}\|f\|_{q}^{q} \leq\|S \mathbf{b}\|_{\ell_{q, m}}^{q} \leq C_{2}\|f\|_{q}^{q}, \quad \forall \mathbf{b}=\left(b_{1}, \ldots, b_{N}\right)
$$


where for $\mathbf{y}=\left(y^{1}, \ldots, y^{m}\right) \in \mathbb{C}^{m}$

$$
\|\mathbf{y}\|_{\ell_{q, m}}:=\left(\frac{1}{m} \sum_{j=1}^{m}\left|y^{j}\right|^{q}\right)^{1 / q} .
$$

Assume now that all $\psi_{i}$ are piecewise constant and the set $\Omega$ can be split $\Omega=\sqcup_{j=1}^{M} \omega_{j}, \mu\left(\omega_{j}\right)=1 / M, 1 \leq j \leq M$, in such a way that

$$
\psi_{i}(\mathbf{x})=c_{i}^{j}, \quad \mathbf{x} \in \omega_{j} .
$$

Denote $F:=\left[c_{i}^{j}\right], j=1, \ldots, M, i=1, \ldots, N$. It is clear that $\|F \mathbf{b}\|_{\ell_{q, M}}=\|f\|_{q}$. Therefore, inequalities (1.5) mean that we are looking for a submatrix $S$ of the full matrix $F$, which represents it well, namely:

$$
C_{1}\|F \mathbf{b}\|_{q}^{q} \leq\|S \mathbf{b}\|_{\ell_{q, m}}^{q} \leq C_{2}\|F \mathbf{b}\|_{q}^{q}, \quad \forall \mathbf{b}=\left(b_{1}, \ldots, b_{N}\right) \in \mathbb{C}^{N}
$$

For applications, the most important case is discretization of the $L_{2}$ norm in subspaces of $L_{2}(\Omega)$. In this case we consider an orthonormal basis $\left\{\psi_{i}\right\}_{i=1}^{N}$ of a given subspace and study the spectral properties of matrices of the form

$$
\left[\psi_{i}\left(\xi^{j}\right)\right], \quad \xi=\left\{\xi^{j}\right\} \subset \Omega \text {. }
$$

For what follows, we need to introduce the $(p, q)$-norm of a matrix: For an $M \times N$ matrix $A$ and $1 \leq p, q \leq \infty$ define

$$
\|A\|_{(p, q)}=\sup _{\|\mathbf{x}\|_{\ell_{p}^{N}} \leq 1}\|A \mathbf{x}\|_{\ell_{q}^{M}}
$$

For brevity in the case $p=q=2$, we denote $\|A\|_{(2,2)}$ simply as $\|A\|$. We present known estimates for the $(p, q)$-norms of submatrices (and related quantities) of a given matrix in Section 3.

In a number of applications it is not required to have two-sided inequalities (1.4), it is sufficient to obtain one-sided estimates of the form

$$
\|f\|_{q}^{q} \geq C \sum_{j=1}^{m} \lambda_{j}\left|f\left(\xi^{j}\right)\right|^{q}, \quad \forall f \in X_{N}
$$

or

$$
\|f\|_{q}^{q} \leq C \sum_{j=1}^{m} \lambda_{j}\left|f\left(\xi^{j}\right)\right|^{q}, \quad \forall f \in X_{N} .
$$


One example when we need to know one-sided estimates of the form (1.7) is the Large Sieve method in number theory. It is based on upper estimates of the $(2,2)$-norms of submatrices of the form

$$
\left[e^{i k \xi_{j}}\right], \quad 1 \leq k \leq n, \quad \xi=\left\{\xi_{j}\right\}_{j=1}^{m} \subset(0,2 \pi)
$$

in terms of properties of the set $\xi$ (see [108, Ch.2] for details). Another example of application of (only) one-sided estimates (1.7) is Marcinkiewicz's proof of convergence in the $L_{p}, 1 \leq p<\infty$, of interpolation polynomials for continuous functions (see [98]). Inequalities of the form (1.8) are useful for estimating conditional numbers of the corresponding matrices when applying Galerkin's method.

The first estimates of the norms of submatrices of a general matrix were established by Kashin in [66] and [67] in connection with A. N. Kolmogorov's problem (still unsolved) on permutations of orthonormal systems. It was proved in [66] (see M.1 below) that every matrix $A$ with $\|A\|=1$ and big enough $M / N$ contains an $N \times N$ submatrix with small $(2,2)-$ norm. At the first step of the proof the estimates of $(2,1)$-norms of random $2 N \times N$ submatrices of $A$ were established. At the second step of the proof the Grothendieck factorization theorem on operators acting from $\ell_{2}^{N}$ to $\ell_{1}^{2 N}$ was used. Sharp estimates in the mentioned above theorem on submatrices were obtained by Lunin [97]. This direction was further developed in the series of works by Bourgain and Tzafriri (see [21], [22]), where the remarkable restricted invertibility theorem M.2, M.3 (see the survey [111] about it) was proved: Let $A$ be an $N \times N$ matrix with ones on the diagonal, then there is a submatrix of $A$ whose rank is proportional to that of $A$ and which is well invertible in the sense that the norm of the inverse matrix depends only on the norm of $A$ and does not depend on the dimension $N$ (see M.3, M.13). A probabilistic selection based on a decoupling principle and a factorization argument were used here. The discussed result has important applications in harmonic analysis and in the study of hilbertian systems (see [22]). The method from the paper [23] of averaging of the norms of submatrices of a given size (raised to the power of $\log N$ ) gave a partial solution of the old Kadison-Singer problem from quantum mechanics [61], which has been finally solved by Marcus, Spielman, and Srivastava in [99].

A new efficient method for finding both upper and lower bounds for the norms of submatrices was proposed by Batson, Spielman, and Srivastava in the paper [5]. They considered positive semidefinite matrices $A$ of rank $N$ 
written as a sum of outer products

$$
A=\sum_{i \leq M} v_{i} v_{i}^{T}
$$

where the number of terms $M$ may be much larger than the rank $N$. Such a representation arises from any $N \times M$ matrix $B$ with columns $b_{i}$ by considering $A=B B^{T}=\sum_{i<M} b_{i} b_{i}^{T}$. It is shown in [5] that for any $N \times M$ matrix $B$ and $\varepsilon>0$, there is a nonnegative diagonal $M \times M$ matrix $S$ with at most $\left\lceil N / \varepsilon^{2}\right\rceil$ nonzero entries such that $(1-\varepsilon)^{2} B B^{T} \preceq B S B^{T} \preceq(1+\varepsilon)^{2} B B^{T}$. We comment on this result at the end of Subsection 4.3. The recently developed method of interlacing polynomials by Marcus, Spielman, and Srivastava led to a number of important results and allowed, in particular, to obtain a solution to the Kadison-Singer problem in [99] (see M.7 for another formulation):

Theorem 1.1. Let a system of vectors $\mathbf{v}_{1}, \ldots, \mathbf{v}_{M}$ from $\mathbb{C}^{N}$ have the following properties: for all $\mathbf{w} \in \mathbb{C}^{N}$

$$
\sum_{j=1}^{M}\left|\left\langle\mathbf{w}, \mathbf{v}_{j}\right\rangle\right|^{2}=\|\mathbf{w}\|_{2}^{2}
$$

and for some $\varepsilon>0$

$$
\left\|\mathbf{v}_{j}\right\|_{2} \leq \varepsilon, \quad j=1, \ldots, M
$$

Then there is a partition of $\{1,2, \ldots, M\}$ into two sets $S_{1}$ and $S_{2}$ such that for all $\mathbf{w} \in \mathbb{C}^{N}$ and for each $i=1,2$

$$
\sum_{j \in S_{i}}\left|\left\langle\mathbf{w}, \mathbf{v}_{j}\right\rangle\right|^{2} \leq \frac{(1+\varepsilon \sqrt{2})^{2}}{2}\|\mathbf{w}\|_{2}^{2} .
$$

Note that condition (1.9) means that $\sum_{j \leq M} \mathbf{v}_{j} \overline{\mathbf{v}}_{j}^{T}$ is the $N \times N$ identity matrix. The main application of Theorem 1.1 in view of this survey is that it leads to the final in the sense of order Marcinkiewicz-type discretization theorem in $L_{2}$ (see D.15 and T.4). Theorem 1.1 has found many other applications. For example, it is used for the estimation of the effectiveness of computational methods ([119]), see M.8 below. In graph theory it is related to sparsification and used to find a small subgraph of a graph with similar spectral properties (see, e.g., [145] and [142]). The proof of existence of 
exponential frames in $L_{2}(S)$ for every unbounded set $S$ of finite measure in $\mathbb{R}$ (see [109]) is also based on Theorem 1.1. We refer the reader to the survey [24] on a recent progress on continuous frames inspired by the solution to the Kadison-Singer problem. The method of [5] was used to construct one feature selection technique for linear Support Vector Machine, see [121].

The above discussed topic is related to the results on the number of contact points of a convex body with its John ellipsoid (see, e.g. [128], [168], and [144] for algorithmic results). We have listed here only some possible applications of the results on the spectral and related properties of submatrices.

The scope of applications of results on the estimates of the operator norms and other properties of submatrices of a given matrix, considered in Section 3, is not limited to discretization problems. In turn, this topic belongs to a wider range of problems on the restriction of operators to coordinate subspaces. The problem here is formulated as follows. Let $X, Y$ be the Banach spaces, $T: X \rightarrow Y$ be a linear operator, $\left\{\varphi_{j}\right\}_{j \in \Omega}$ where $\Omega=\mathbb{N}$ (in the case of infinite-dimensional $X$ ) or $\Omega=\{1, \ldots, N\}, N \in \mathbb{N}$ (for finite-dimensional $X$ ) be a basis of $X$. We are looking for a subspace $L \subset X$ of the form $L=\operatorname{span}_{j \in \Gamma}\left\{\varphi_{j}\right\}, \Gamma \subset \Omega$, such that the restriction of the operator $T$ on the subspace $L$ has additional properties. The dual formulation of the problem uses a basis $\left\{f_{j}\right\}$ of $Y$. In that case we want to find $\Gamma \subset \mathbb{N}$ such that the operator $\left.T\right|_{L_{1}}, L_{1}=\operatorname{span}_{j \in \Gamma}\left\{f_{j}\right\}$, has additional properties. As an example let us formulate the Banach theorem (see, e.g. [62]): Let $\left\{\psi_{j}\right\}_{j=1}^{\infty}$ be an orthonormal system with the following property: for some $p>2$ and $D>0$ $\left\|\psi_{j}\right\|_{p} \leq D, j \in \mathbb{N}$. Then there exists an infinite subsystem $\left\{\psi_{\nu}\right\}_{\nu \in \Gamma}$ so that for all $\left\{a_{\nu}\right\}_{\nu \in \Gamma} \subset \mathbb{R}$ the following inequality holds:

$$
\left\|\sum_{\nu \in \Gamma} a_{\nu} \psi_{\nu}\right\|_{L_{p}} \leq C_{D, p}\left(\sum_{\nu \in \Gamma} a_{\nu}^{2}\right)^{1 / 2}
$$

In this case $X=\ell_{2}, Y=L_{2}, T: \ell_{2} \rightarrow L_{2}, T\left(\left\{a_{j}\right\}_{j=1}^{\infty}\right)=\sum_{j=1}^{\infty} a_{j} \psi_{j}$. Banach's theorem means that the operator $T$ acts between $\ell_{2}$ and $L_{p}$ on the coordinate subspace $\operatorname{span}\left\{e_{\nu}, \nu \in \Gamma\right\}$ of $\ell_{2}$. In the finite-dimensional case, the largest dimension of the corresponding good subspace is usually of interest. We recall, for example, a deep result by Bourgain [16]: Under the additional assumption $\left\|\psi_{j}\right\|_{L_{\infty}} \leq D, 1 \leq j \leq N$, we can choose a subsystem $\left\{\psi_{j}\right\}_{j \in \Gamma}$ from an orthonormal system $\left\{\psi_{j}\right\}_{j=1}^{N}$ with $|\Gamma| \geq N^{2 / p}$ such that (1.10) holds. 
This theorem can be seen as a finite-dimensional analog of Banach's theorem above.

Connections with other areas. In Section 4 we discuss connections between the sampling discretization problem and other areas of research and describe how ideas and techniques from these areas can be applied in the study of sampling discretization.

Moments of random vectors. In Subsection 4.1 we discuss the probabilistic approach to sampling discretization of the $L_{q}$ norms of elements of an $N$-dimensional subspace $X_{N}$. For $f \in X_{N}$ the quantity

$$
\operatorname{er}\left(f, \xi, L_{q}\right):=\left.\left|\|f\|_{q}^{q}-\frac{1}{m} \sum_{j=1}^{m}\right| f\left(\xi^{j}\right)\right|^{q} \mid, \quad \xi=\left(\xi^{1}, \ldots, \xi^{m}\right),
$$

can be seen as a random variable on $\Omega^{m}$ with the measure $\mu^{m}$. Let a system of functions $\left\{u_{i}(x)\right\}_{i=1}^{N}, x \in \Omega$, form a basis of $X_{N}$. Consider the vector function $\mathbf{u}(x):=\left(u_{1}(x), \ldots, u_{N}(x)\right)$, which can be interpreted as a random vector on the probability space $(\Omega, \mu)$ and consider $m$ independent copies $\mathbf{u}^{j}:=\mathbf{u}\left(\xi^{j}\right)$ of this random vector. We associate with $f=\sum_{i=1}^{N} y_{i}(f) u_{i}$ a vector of its coefficients $\mathbf{y}(f)=\left(y_{1}(f), \ldots, y_{N}(f)\right)$. Then a class $\mathbf{F} \subset X_{N}$ of functions can be described by the class $K(\mathbf{F}):=\{\mathbf{y}(f): f \in \mathbf{F}\} \subset \mathbb{R}^{N}$ of their coefficients and vice versa for $K \subset \mathbb{R}^{N}$ define $\mathbf{F}(K):=\left\{f: \sum_{i=1}^{N} y_{i} u_{i}, \mathbf{y} \in K\right\}$.

For a set $K \subset \mathbb{R}^{N}$ we consider the following random variable

$$
V_{q}(K):=\left.\sup _{\mathbf{y} \in K}\left|\frac{1}{m} \sum_{j=1}^{m}\right|\left\langle\mathbf{y}, \mathbf{u}^{j}\right\rangle\right|^{q}-\mathbb{E}|\langle\mathbf{y}, \mathbf{u}\rangle|^{q} \mid,
$$

where $\langle\mathbf{y}, \mathbf{x}\rangle=y_{1} x_{1}+\cdots+y_{N} x_{N}$ for $\mathbf{x}, \mathbf{y} \in \mathbb{R}^{N}$ and $\mathbb{E}$ denotes the expectation of a random variable. Clearly,

$$
V_{q}(K)=\operatorname{er}\left(\mathbf{F}(K), \xi, L_{q}\right):=\sup _{f \in \mathbf{F}(K)} \operatorname{er}\left(f, \xi, L_{q}\right) .
$$

In the sampling discretization we are interested in dependence of bounds for $\inf _{\xi} \operatorname{er}\left(\mathbf{F}(K), \xi, L_{q}\right)$ on the number $m$ of sampling points. If now one obtains bounds for the expectation $\mathbb{E} V_{q}(K)$ or for the probability of the event $\left\{V_{q}(K)>\varepsilon\right\}$, then clearly as a corollary one gets a result about sampling discretization. Thus, the following general problem arises: How many independent copies of a random vector $\mathbf{u}$ are needed to get $V_{q}(K) \leq \varepsilon$ with high probability. 
Such a probabilistic reformulation of the initial problem proved to be useful in many areas. The first results on approximation of the moments of random vectors were actually motivated by the study of problems of complexity in computing volumes in high dimensions (see [17], [64]), by the study of restrictions of operators to coordinate subspaces (see [129], where the problem proposed by B. Kashin and L. Tzafriri in [79] was discussed), and by the study of the embedding of finite-dimensional subspaces $X_{N}$ of $L_{q}$ into $\ell_{q}^{m}$ (see [149] and [150]).

Embedding of finite-dimensional subspaces. In Subsection 4.2 we discuss the problem of a good (almost isometrical) embedding of an $\mathrm{N}$-dimensional subspace $X_{N}$ of $L_{q}[0,1]$ into an $N$-dimensional subspace of $\ell_{q}^{m}$ with the smallest possible $m$. This type of problem was originally motivated by the study of the bounds for the dimension of an almost spherical section in the Dvoretzky theorem (see [41] and [65], or Chapter 5 in [3]). In [59] and [131] embeddings of general spaces into $\ell_{1}^{m}$ were studied. Finally, in [132], the described above problem appeared. In this problem researchers do not restrict themselves to embedding by sampling and are allowed to use any linear embedding. Nevertheless, almost all approaches to this problem go through sampling (the empirical method). In Subsection 4.2 we describe several methods for solving the problem of embedding, which can be applied in sampling discretization.

Sparse approximation. In Subsection 4.3 we give some remarks, which illustrate a connection between sparse approximation and sampling discretization of the $L_{2}$ norm. The properties of the subspace $X_{N}$ can be described in terms of a system $\mathcal{U}_{N}:=\left\{u_{i}\right\}_{i=1}^{N}$ of functions such that $X_{N}:=\operatorname{span}\left\{u_{i}\right\}_{i=1}^{N}$. In the case $X_{N} \subset L_{2}$ we assume that the system $\mathcal{U}_{N}$ is orthonormal on $\Omega$ with respect to the measure $\mu$. In the case of real functions we associate with $x \in \Omega$ the matrix $G(x):=\left[u_{i}(x) u_{j}(x)\right]_{i, j=1}^{N}$. Clearly, $G(x)$ is a symmetric positive semi-definite matrix of rank $\leq 1$. It is easy to see that for a set of points $\xi^{k} \in \Omega, k=1, \ldots, m$, and $f=\sum_{i=1}^{N} b_{i} u_{i}$ we have

$$
\sum_{k=1}^{m} \lambda_{k} f\left(\xi^{k}\right)^{2}-\int_{\Omega} f(x)^{2} d \mu=\mathbf{b}^{T}\left(\sum_{k=1}^{m} \lambda_{k} G\left(\xi^{k}\right)-I\right) \mathbf{b},
$$

where $\mathbf{b}=\left(b_{1}, \ldots, b_{N}\right)^{T}$ is the column vector and $I$ is the identity matrix. Therefore, the sampling discretization problem is closely connected with a problem of approximation (representation) of the identity matrix $I$ by an $m$-term approximant with respect to the system $\{G(x)\}_{x \in \Omega}$. We can obtain 
some results on this approximation problem by greedy approximation methods, which provide a constructive way of finding good sampling points and by applying results from the theory of random matrices. We discuss these methods in more detail in Subsection 4.3.

Learning theory. In Subsection 4.4 we demonstrate that the sampling discretization problem of the $L_{2}$ norm is closely related to supervised learning theory. This is a vast area of research with a wide range of different settings. In Subsection 4.4 we only discuss a development of a setting from [30] (see [153], Ch.4, for detailed discussion). In our further discussion we are interested in discretization of the $L_{2}$ norm of real functions from a given function class $\mathbf{F}$. It is well known in learning theory that performance of the empirical risk minimization algorithm (least squares) can be controlled by asymptotic characteristics (entropy, Kolmogorov widths) measured in the uniform norm. Typically, analysis there is based on Bernstein's concentration measure inequality and chaining techniques.

Let $\Omega \subset \mathbb{R}^{d}, Y \subset \mathbb{R}$ be Borel sets, $\rho$ be a Borel probability measure on a Borel set $Z \subset \Omega \times Y$. For $f: \Omega \rightarrow Y$ define the error

$$
\mathcal{E}(f):=\int_{Z}(f(\mathbf{x})-y)^{2} d \rho
$$

Let $\rho_{\Omega}$ be the marginal probability measure of $\rho$ on $\Omega$, i.e. for any Borel set $S \subset \Omega$ one has $\rho_{\Omega}(S)=\rho(S \times Y)$. Define

$$
f_{\rho}(\mathbf{x}):=\mathbb{E}(y \mid \mathbf{x})
$$

to be a conditional expectation of $y$. The function $f_{\rho}$ is known in statistics as the regression function of $\rho$. It is clear that if $f_{\rho} \in L_{2}\left(\rho_{\Omega}\right)$, then it minimizes the error $\mathcal{E}(f)$ over all $f \in L_{2}\left(\rho_{\Omega}\right)$ i.e. $\mathcal{E}\left(f_{\rho}\right) \leq \mathcal{E}(f)$. Thus, in the sense of error $\mathcal{E}(\cdot)$ the regression function $f_{\rho}$ is the best to describe the relation between inputs $\mathbf{x} \in \Omega$ and outputs $y \in Y$. The goal is to find an estimator $f_{\mathbf{z}}$, on the base of given data $\mathbf{z}:=\left(\left(\mathbf{x}^{1}, y_{1}\right), \ldots,\left(\mathbf{x}^{m}, y_{m}\right)\right)$ that approximates $f_{\rho}$ well with high probability. We assume that $\left(\mathbf{x}^{i}, y_{i}\right), i=1, \ldots, m$, are independent and distributed according to $\rho$. We measure the error between $f_{\mathbf{z}}$ and $f_{\rho}$ in the $L_{2}\left(\rho_{\Omega}\right)$ norm.

We note that a standard setting in the distribution-free theory of regression (see [54]) involves the expectation $\mathbb{E}\left(\left\|f_{\rho}-f_{\mathbf{z}}\right\|_{L_{2}\left(\rho_{\Omega}\right)}^{2}\right)$ as a measure of quality of an estimator. An important new feature of the setting in learning theory formulated in [30] (see [153] for detailed discussion) is the following. 
We begin with a class $\mathcal{M}$ of admissible measures $\rho$. Usually, we impose restrictions on $\rho$ in the form of restrictions on the regression function $f_{\rho} \in \Theta$, where $\Theta$ is a given class of priors. Then the first step is to find an optimal estimator for $\Theta$. In regression theory we typically evaluate the performance of an estimator $f_{\mathbf{z}}$ by studying its convergence in expectation, i.e. the rate of decay of the quantity $\mathbb{E}\left(\left\|f_{\rho}-f_{\mathbf{z}}\right\|_{L_{2}\left(\rho_{\Omega}\right)}^{2}\right)$ as the sample size $m$ increases. Here the expectation is taken with respect to the product measure $\rho^{m}$ defined on $Z^{m}$. We note that $\mathcal{E}\left(f_{\mathbf{z}}\right)-\mathcal{E}\left(f_{\rho}\right)=\left\|f_{\mathbf{z}}-f_{\rho}\right\|_{L_{2}\left(\rho_{\Omega}\right)}^{2}$. A more accurate and more refined way of evaluating the performance of $f_{\mathbf{z}}$ was proposed in [30]: to systematically study the probability distribution function

$$
\rho^{m}\left\{\mathbf{z}:\left\|f_{\rho}-f_{\mathbf{z}}\right\|_{L_{2}\left(\rho_{\Omega}\right)} \geq \eta\right\}
$$

instead of the expectation.

We define the empirical error of $f$ as

$$
\mathcal{E}_{\mathbf{z}}(f):=\frac{1}{m} \sum_{i=1}^{m}\left(f\left(\mathbf{x}^{i}\right)-y_{i}\right)^{2} .
$$

Let $f \in L_{2}\left(\rho_{\Omega}\right)$. The defect function of $f$ is

$$
L_{\mathbf{z}}(f):=L_{\mathbf{z}, \rho}(f):=\mathcal{E}(f)-\mathcal{E}_{\mathbf{z}}(f) ; \quad \mathbf{z}=\left(z_{1}, \ldots, z_{m}\right), \quad z_{i}=\left(\mathbf{x}^{i}, y_{i}\right) .
$$

We are interested in estimating the supremum of $\left|L_{\mathbf{z}}(f)\right|$ over functions $f$ coming from a given class.

Settings for a supervised learning problem and a discretization problem are different. In the supervised learning problem we are given a sample $\mathbf{z}$ and we want to approximately recover the regression function $f_{\rho}$. It is important that we do not know $\rho$. We only assume that we know that $f_{\rho} \in \Theta$. In the discretization of the $L_{q}, 1 \leq q<\infty$, norm we assume that $f \in \mathbf{F}$ and the probability measure $\mu$ is known. We want to find a discretization set $\xi=\left\{\mathbf{x}^{j}\right\}_{j=1}^{m}$, which is good for the whole class $\mathbf{F}$. However, the technique, based on the defect function, for solving the supervised learning problem can be applied to the discretization problem. Let us consider a given function class $\mathbf{F}$ of real functions, defined on $\Omega$. Suppose that the probability measure $\rho$ is such that $\rho_{\Omega}=\mu$ and for all $\mathbf{x} \in \Omega$ we have $y=0$. In other words, we assume that $Y=\{0\}$. Then for the defect function we have

$$
L_{\mathbf{z}}(f)=\int_{\Omega} f^{2} d \mu-\frac{1}{m} \sum_{j=1}^{m} f\left(\mathbf{x}^{j}\right)^{2}, \quad\left|L_{\mathbf{z}}(f)\right|=\operatorname{er}\left(f,\left(\mathbf{x}^{1}, \ldots, \mathbf{x}^{m}\right), L_{2}\right)
$$


and

$$
\rho^{m}\left\{\mathbf{z}: \sup _{f \in \mathbf{F}}\left|L_{\mathbf{z}}(f)\right| \geq \eta\right\}=\mu^{m}\left\{\mathbf{w} \in \Omega^{m}: \sup _{f \in \mathbf{F}} \operatorname{er}\left(f, \mathbf{w}, L_{2}\right) \geq \eta\right\} .
$$

In Subsection 4.4 we illustrate this connection by highlighting some known results from learning theory and demonstrating how those results imply results on sampling discretization.

The technique used there is a probabilistic technique. We derive sampling discretization results from the estimates of $\rho^{m}\left\{\mathbf{z}: \sup _{f \in \mathbf{F}}\left|L_{\mathbf{z}}(f)\right| \leq \varepsilon\right\}$. We stress that in order to prove existence of a point set that is good for the whole class we study the probability distribution of the supremum of the random quantity of interest over the class. There is other approach of using randomized algorithms (see, for instance, [57]). In this approach we first take the expectation of the random quantity of interest for each individual function in the class and after that we take the supremum over the whole class. This approach is similar to the Monte Carlo approach for numerical integration discussed in Subsection 4.4. However, as it is pointed out in Subsection 4.4, it does not help to obtain results for the whole class, which contains infinitely many functions. It is an interesting and important setting but it is not directly related to our setting of looking for a good point set for the whole class.

The structure of the paper. We now comment on the further structure of the paper. Section 2 contains results on sampling discretization of integral norms $L_{q}, 1 \leq q<\infty$, and the uniform norm $L_{\infty}$ of elements of finitedimensional subspaces. In Subsection 2.1 we present results on discretization of norms of trigonometric polynomials, in Subsection 2.2 we discuss general subspaces, and in Subsections 2.3 and 2.4 - algebraic polynomials. Mostly, these subsections contain known results, which we formulate in paragraphs enumerated by letter $\mathbf{D}$ with a number. Letter $\mathbf{D}$ stands for Discretization. In Subsection 2.5 we present a brief discussion of recent results on sampling recovery proved with the aid of sampling discretization results. We enumerate the corresponding results by letter $\mathbf{R}$ with a number. Letter $\mathbf{R}$ stands for Recovery. In Subsection 2.6 we complement the discussion on techniques from Section 1 by more detailed discussion of important techniques used in sampling discretization. We enumerate the corresponding results by letter $\mathbf{T}$ with a number. Letter $\mathbf{T}$ stands for Technique. Finally, in Subsection 2.7 we formulate some open problems. We note that the material of Section 2 complements the earlier survey on sampling discretization [33]. 
In Section 3 we give a survey on results about the operator $(p, q)$-norms of submatrices which are connected to discretization. In addition to the classical case of the $(2,2)$-norm (spectral norm) we pay attention to the case of arbitrary $1 \leq p, q \leq \infty$, which is not sufficiently covered in the literature.

Section 4 is devoted to a detailed discussion of connections between settings and results from sampling discretization with settings and results from other areas of mathematics. We show that the corresponding connections are very close. It is a new and important contribution of this survey. In Subsection 4.1 we demonstrate that the sampling discretization problem is very close to the corresponding problem on approximation of moments of marginals of high-dimensional distributions. We also show how several results about high-dimensional random vectors can be applied to sampling discretization. In Subsection 4.1 we split the material into three large pieces enumerated by MM.1 - MM.3 and formulate results in terms of theorems and corollaries. In Subsection 4.2 we discuss the problem of good embedding of an $N$-dimensional subspace $X_{N}$ of $L_{q}$ into $\ell_{q}^{m}$ with the smallest possible $m$. In this subsection we present the best known results concerning the described problem (Em.1) and then we give some ideas, that are used in the proofs of these results and can also be useful in the sampling discretization problem (Em.2 - Em.6). In Subsection 4.3 we give a brief comment on the connection between sampling discretization of the $L_{2}$ norm and the $m$-term approximation of the identity matrix with respect to a special dictionary, determined by the subspace $X_{N}$, of matrices of rank one. In Subsection 4.4 we demonstrate how known results from learning theory can be used for establishing upper bounds for the sampling discretization error $e r_{m}\left(\mathbf{F}, L_{2}\right)$ for certain function classes $\mathbf{F}$. We formulate results of this subsection in a form of theorems and corollaries.

Occasionally, for the reader's convenience we write $a_{m} \ll b_{m}$ instead of $a_{m} \leq C b_{m}$, where $C$ is a positive constant independent of $m$. In case $a_{m} \ll b_{m}$ and $b_{m} \ll a_{m}$ we write $a_{m} \asymp b_{m}$.

\section{Discretization}

We give some general remarks before we proceed to technical details. We are interested in discretization of the $L_{q}$ norms, $1 \leq q \leq \infty$, of elements of finitedimensional subspaces. If a subspace of interest is a subspace of $\mathcal{C}(\Omega)$, then all the functions from this subspace are defined at each point of $\Omega$. This is the 
case in many concrete situations, for instance, in the case of trigonometric and algebraic polynomials. However, we encounter a problem when we want to consider arbitrary subspaces of $L_{q}, 1 \leq q<\infty$. In this case by a function $f \in L_{q}(\Omega, \mu)$ we understand a specific function (not an equivalency class), which is defined almost everywhere with respect to $\mu$ on $\Omega$. In other words, for $f \in L_{q}(\Omega, \mu)$ there exists a set $E(f) \subset \Omega$ such that $\mu(E(f))=0$ and $f(x)$ is defined for all $x \in \Omega \backslash E(f)$. We say that a subspace $X_{N} \subset L_{q}(\Omega, \mu)$ is an $N$-dimensional subspace (more precisely, has dimension $\leq N$ ) if there are $N$ functions $u_{i} \in X_{N}, i=1, \ldots, N$, such that $X_{N}=\operatorname{span}\left\{u_{1}, \ldots, u_{N}\right\}$. In this case, for a subspace $X_{N}$ there exists a set $E\left(X_{N}\right) \subset \Omega$ such that $\mu\left(E\left(X_{N}\right)\right)=0$ and each $f \in X_{N}$ is defined for all $x \in \Omega \backslash E\left(X_{N}\right)$.

The Marcinkiewicz discretization problem. Let $\Omega$ be a compact subset of $\mathbb{R}^{d}$ with the probability measure $\mu$. We say that a linear subspace $X_{N}$ (index $N$ here, represents the dimension of $X_{N}$ ) of $L_{q}(\Omega, \mu), 1 \leq q<\infty$, admits the Marcinkiewicz-type discretization theorem with parameters $q$ and $m \in \mathbb{N}$ and positive constants $C_{1} \leq C_{2}$ if there exists a set

$$
\left\{\xi^{j} \in \Omega: j=1, \ldots, m\right\}
$$

such that for any $f \in X_{N}$ we have

$$
C_{1}\|f\|_{q}^{q} \leq \frac{1}{m} \sum_{j=1}^{m}\left|f\left(\xi^{j}\right)\right|^{q} \leq C_{2}\|f\|_{q}^{q} .
$$

The Bernstein discretization problem. In the case $q=\infty$ we define $L_{\infty}$ as the space of continuous functions on $\Omega$ and ask for

$$
C_{1}\|f\|_{\infty} \leq \max _{1 \leq j \leq m}\left|f\left(\xi^{j}\right)\right| \leq\|f\|_{\infty}
$$

We will also use the following notation to express the above properties: The $\mathcal{M}(m, q)$ (more precisely the $\mathcal{M}\left(m, q, C_{1}, C_{2}\right)$ ) theorem holds for a subspace $X_{N}$, written $X_{N} \in \mathcal{M}(m, q)$ (more precisely $X_{N} \in \mathcal{M}\left(m, q, C_{1}, C_{2}\right)$ ). In the case $q=\infty$ we write $\mathcal{M}\left(m, \infty, C_{1}\right)$.

The Marcinkiewicz discretization problem with weights. We say that a linear subspace $X_{N}$ of the $L_{q}(\Omega, \mu), 1 \leq q<\infty$, admits the weighted Marcinkiewicz-type discretization theorem with parameters $m$ and $q$ and positive constants $C_{1} \leq C_{2}$ if there exist a set of knots $\left\{\xi^{\nu} \in \Omega\right\}$ and a set of 
weights $\left\{\lambda_{\nu} \in \mathbb{R}\right\}, \nu=1, \ldots, m$, such that for any $f \in X_{N}$ we have

$$
C_{1}\|f\|_{q}^{q} \leq \sum_{\nu=1}^{m} \lambda_{\nu}\left|f\left(\xi^{\nu}\right)\right|^{q} \leq C_{2}\|f\|_{q}^{q} .
$$

Then we also say that the $\mathcal{M}^{w}(m, q)$ (more precisely the $\mathcal{M}^{w}\left(m, q, C_{1}, C_{2}\right)$ ) theorem holds for a subspace $X_{N}$ and write $X_{N} \in \mathcal{M}^{w}(m, q)$ (more precisely, $\left.X_{N} \in \mathcal{M}^{w}\left(m, q, C_{1}, C_{2}\right)\right)$. Obviously, $X_{N} \in \mathcal{M}(m, q)$ implies that $X_{N} \in \mathcal{M}^{w}(m, q)$.

The Marcinkiewicz discretization problem with $\varepsilon$. For $1 \leq q<\infty$ we write $X_{N} \in \mathcal{M}(m, q, \varepsilon)$ if $(2.1)$ holds with $C_{1}=1-\varepsilon$ and $C_{2}=1+\varepsilon$. Respectively, we write $X_{N} \in \mathcal{M}^{w}(m, q, \varepsilon)$ if (2.2) holds with $C_{1}=1-\varepsilon$ and $C_{2}=1+\varepsilon$.

In the case, when we need to specify either the set $\Omega$ or the measure $\mu$ we write in the notation $L_{q}(\Omega, \mu)$ instead of $q$, for instance, $\mathcal{M}^{w}\left(m, L_{q}(\Omega, \mu)\right)$ instead of $\mathcal{M}^{w}(m, q)$. We note that the most powerful results are when the $L_{q}$ norm of $f \in X_{N}$ is discretized exactly by the formula with equal weights $1 / m$, i.e. the $\mathcal{M}(m, q, 0)$ theorems.

The optimal in the sense of order sampling discretization results are those, which guarantee $X_{N} \in \mathcal{M}\left(C N, q, C_{1}, C_{2}\right)$. This is closely related to the following concept of quasi-matrix systems introduced in [69] in the case of $p=\infty$.

Definition 2.1. Let $p \in[1, \infty]$. A system of functions $\Phi=\left\{\varphi_{j}(x)\right\}_{j=1}^{\infty}$, $x \in \Omega$, is called quasi-matrix if there exist positive constants $K_{i}, i=1,2,3$, such that for any $N \in \mathbb{N}$ there is a finite set $\left\{\xi^{j}\right\}_{j=1}^{m} \subset \Omega$ with $m \leq K_{1} N$ with the property: For any $f=\sum_{j=1}^{N} a_{j} \varphi_{j}$ the following inequalities hold in the case $1 \leq p<\infty$

$$
K_{2}\|f\|_{p} \leq\left(\frac{1}{m} \sum_{j=1}^{m}\left|f\left(\xi^{j}\right)\right|^{p}\right)^{1 / p} \leq K_{3}\|f\|_{p}
$$

and for $p=\infty$

$$
K_{2}\|f\|_{\infty} \leq \max _{1 \leq j \leq m}\left|f\left(\xi^{j}\right)\right|
$$

\subsection{Trigonometric polynomials}

In this subsection we specify $\Omega:=\mathbb{T}^{d}:=[0,2 \pi)^{d}$ and $\mu$ to be the normalized Lebesgue measure $d \mu=(2 \pi)^{-d} d \mathbf{x}$. By $Q$ we denote a finite subset of $\mathbb{Z}^{d}$, and 
$|Q|$ stands for the number of elements in $Q$. Let

$$
\mathcal{T}(Q):=\left\{f: f=\sum_{\mathbf{k} \in Q} c_{\mathbf{k}} e^{i\langle\mathbf{k}, \mathbf{x}\rangle}, \quad c_{\mathbf{k}} \in \mathbb{C}\right\} .
$$

Polynomials with frequencies from parallelepipeds. Consider $d$ dimensional parallelepipeds

$$
\Pi(\mathbf{N}, d):=\left\{\mathbf{a} \in \mathbb{Z}^{d}:\left|a_{j}\right| \leq N_{j}, j=1, \ldots, d\right\}, \quad \mathbf{N}=\left(N_{1}, \ldots, N_{d}\right),
$$

where $N_{j}$ are nonnegative integers and the corresponding subspaces of the trigonometric polynomials

$$
\mathcal{T}(\mathbf{N}, d):=\mathcal{T}(\Pi(\mathbf{N}, d)) .
$$

Then $\operatorname{dim} \mathcal{T}(\mathbf{N}, d)=\prod_{j=1}^{d}\left(2 N_{j}+1\right)$.

D.1. The following classical result on exact discretization of the $L_{2}$ norm is well known (see, for instance, [157], p.7)

$$
\mathcal{T}(\mathbf{N}, d) \in \mathcal{M}(\operatorname{dim} \mathcal{T}(\mathbf{N}, d), 2,0) .
$$

D.2. The following result was obtained by Bernstein (see, for instance, [176], Ch.10, Theorem (7.28)) in the case $d=1$

$$
\mathcal{T}(N, 1) \in \mathcal{M}\left((1+\delta) \operatorname{dim} \mathcal{T}(N, 1), \infty, C_{1}(\delta)\right)
$$

D.3. The following result was obtained by Marcinkiewicz in the case $d=1,1<q<\infty$, and by Marcinkiewicz-Zygmund in the case $d=1$, $1 \leq q \leq \infty$ (see [176], Ch.10, §7). For the multivariate analogs see [157], p.102, Theorem 3.3.15.

$$
\mathcal{T}(\mathbf{N}, d) \in \mathcal{M}\left(C(d) \operatorname{dim} \mathcal{T}(\mathbf{N}, d), q, C_{1}(d), C_{2}(d)\right), \quad 1 \leq q \leq \infty .
$$

The reader can find a generalization of the Marcinkiewicz-Zygmund results to the case of weighted $L_{q}$ spaces, namely, to the case of $L_{q}(\mathbb{T}, \mu), d \mu=w(x) d x$, where $w$ is the Muckenhoupt weight, in [81].

Hyperbolic cross polynomials. For $\mathbf{s} \in \mathbb{Z}_{+}^{d}$ define

$$
\rho(\mathbf{s}):=\left\{\mathbf{k} \in \mathbb{Z}^{d}:\left[2^{s_{j}-1}\right] \leq\left|k_{j}\right|<2^{s_{j}}, \quad j=1, \ldots, d\right\}
$$


where $[x]$ denotes the integer part of $x$. We define the step hyperbolic cross $Q_{n}$ as follows

$$
Q_{n}:=\bigcup_{\mathbf{s}:\|\mathbf{s}\|_{1} \leq n} \rho(\mathbf{s})
$$

and the corresponding set of the hyperbolic cross polynomials as

$$
\mathcal{T}\left(Q_{n}\right):=\left\{f: f=\sum_{\mathbf{k} \in Q_{n}} c_{\mathbf{k}} e^{i\langle\mathbf{k}, \mathbf{x}\rangle}\right\}
$$

In addition to the step hyperbolic cross $Q_{n}$ we also consider a more general step hyperbolic cross $Q_{n}^{\gamma}$, where $\gamma=\left(\gamma_{1}, \ldots, \gamma_{d}\right)$ has the form

$$
1=\gamma_{1}=\cdots=\gamma_{\nu}<\gamma_{\nu+1} \leq \cdots \leq \gamma_{d}
$$

with $\nu \in \mathbb{N}, \nu \leq d$ :

$$
Q_{n}^{\gamma}:=\bigcup_{\mathbf{s}:(\gamma, \mathbf{s}) \leq n} \rho(\mathbf{s}), \quad(\gamma, \mathbf{s}):=\gamma_{1} s_{1}+\cdots+\gamma_{d} s_{d} .
$$

It is clear that in the case $\gamma=\mathbf{1}:=(1, \ldots, 1)$ we have $Q_{n}^{\mathbf{1}}=Q_{n}$. Note that $\left|Q_{n}^{\gamma}\right| \asymp 2^{n} n^{\nu-1}$.

D.4. We begin with the case $q=1$. In this case we have (see [159]): There are three positive constants $C_{i}=C_{i}(\gamma), i=1,2,3$, such that we have

$$
\mathcal{T}\left(Q_{n}^{\gamma}\right) \in \mathcal{M}\left(m, 1, C_{1}, C_{2}\right) \quad \text { provided } \quad m \geq C_{3}\left|Q_{n}^{\gamma}\right| n^{3} .
$$

Weaker versions of the above result for $\gamma=\mathbf{1}$ were obtained in [6] (with $n^{3}$ replaced by $n^{4}$ ) and in [154] (with $n^{3}$ replaced by $n^{7 / 2}$ ).

D.5. We continue with the case $q=2$. In this case the problem is solved in the sense of order (see [154]): There are three absolute positive constants $C_{i}, i=1,2,3$, such that we have

$$
\mathcal{T}\left(Q_{n}^{\gamma}\right) \in \mathcal{M}\left(m, 2, C_{1}, C_{2}\right) \quad \text { provided } \quad m \geq C_{3}\left|Q_{n}^{\gamma}\right| .
$$

See D.9 and D.15 for further generalizations.

D.6. Consider the case $1<q<\infty, q \neq 2$. In this case we have (see [159]): There are three positive constants $C_{i}=C_{i}(q, \gamma), i=1,2,3$, such that we have

$$
\mathcal{T}\left(Q_{n}^{\gamma}\right) \in \mathcal{M}\left(m, q, C_{1}, C_{2}\right) \quad \text { provided } \quad m \geq C_{3}\left|Q_{n}^{\gamma}\right| n^{w(\nu, q)}
$$


where

$w(\nu, q)=2, \quad q \in(1,2) ; \quad w(\nu, q)=(\nu-1)(q-2)+\min (q, 3), \quad q \in(2, \infty)$.

A weaker version of the above result for $\gamma=\mathbf{1}:=(1, \ldots, 1)$ with $n^{w(\nu, q)}$ replaced by $n^{\max ((d-1)(q-2), 0)+4}$ was obtained in [6].

D.7. Consider the case $q=\infty$. It turns out that in the case of the hyperbolic cross trigonometric polynomials sampling discretization results involving the $L_{\infty}$ norm are very different from those involving the $L_{q}$ norm, $1 \leq q<\infty$. The following statement was proved in [75] $-[77]$ for $d=2$ : There exists a positive absolute constant $c$ with the property: If $m$ is such that

$$
\mathcal{T}\left(Q_{n}\right) \in \mathcal{M}\left(m, \infty, C_{1}\right), \quad \text { then } \quad m \geq C\left(C_{1}\right)\left|Q_{n}\right|^{1+c} .
$$

D.8. The upper bound for $m$ is given in [33]. Define

$$
\alpha_{d}:=\sum_{j=1}^{d} \frac{1}{j} \quad \text { and } \quad \beta_{d}:=d-\alpha_{d} .
$$

Then there are two positive constants $C_{i}=C_{i}(d), i=1,2$, such that we have

$$
\mathcal{T}\left(Q_{n}\right) \in \mathcal{M}\left(m, \infty, C_{1}\right) \text { provided } m \geq C_{2} 2^{n \alpha_{d}} n^{\beta_{d}} .
$$

General trigonometric polynomials. We now proceed to the case of general trigonometric subspaces of dimension $N$, in other words subspaces $\mathcal{T}(Q)$ with $|Q|=N$.

D.9. We begin with the case $q=2$. The following result was obtained in [154]. There are three absolute positive constants $C_{i}, i=1,2,3$, such that for any $Q \subset \mathbb{Z}^{d}$ we have

$$
\mathcal{T}(Q) \in \mathcal{M}\left(m, 2, C_{1}, C_{2}\right) \text { provided } m \geq C_{3}|Q| .
$$

See D.15 for a further generalization.

D.10. Consider the case $q \in[1,2)$. In this case we have (see [159]): There are three positive constants $C_{i}=C_{i}(q), i=1,2,3$, such that we have for any $Q \in \mathbb{Z}^{d}$

$$
\mathcal{T}(Q) \in \mathcal{M}\left(m, q, C_{1}, C_{2}\right) \quad \text { provided } \quad m \geq C_{3}|Q|(\log (2|Q|))^{w(q)},
$$

where $w(1)=3$ and $w(q)=2$ for $q \in(1,2)$. See D.16 for more general results. 
D.11. We do not have results similar to D.10 in the case $q \in(2, \infty)$. We mention here a corollary of $\mathbf{D . 9}$ for the case of even $q$. Let $q=2 s, s \in \mathbb{N}$. The case $s=1$ is covered by D.9. We consider the case $s \geq 2$. For a given $Q \subset \mathbb{Z}^{d}$ consider the set

$$
Q^{s}:=\left\{\mathbf{k}: \mathbf{k}=\mathbf{k}^{1}+\mathbf{k}^{2}+\cdots+\mathbf{k}^{s}, \mathbf{k}^{j} \in Q, j=1, \ldots, s\right\} .
$$

Then, $\left|Q^{s}\right| \leq|Q|^{s}$ and for any $f \in \mathcal{T}(Q)$ we have $f^{s} \in \mathcal{T}\left(Q^{s}\right)$. Therefore, discretization of the $L_{2 s}$ norm of elements of $\mathcal{T}(Q)$ follows from discretization of the $L_{2}$ norm of elements of $\mathcal{T}\left(Q^{s}\right)$. Applying $\mathbf{D . 9}$ to $\mathcal{T}\left(Q^{s}\right)$ we obtain the following result: There are three absolute positive constants $C_{i}, i=1,2,3$, such that for any $Q \subset \mathbb{Z}^{d}$ we have

$$
\mathcal{T}(Q) \in \mathcal{M}\left(m, 2 s, C_{1}, C_{2}\right) \text { provided } m \geq C_{3}|Q|^{s} .
$$

We also point out (see [86, Corollary 4.5]) that in the case $2<q<\infty$ one has

$$
\mathcal{T}(Q) \in \mathcal{M}(m, q, \varepsilon) \quad \text { provided } \quad m \geq C(q, \varepsilon)|Q|^{\frac{q}{2}} \log |Q| .
$$

See D.17 for more general results.

We point out that bound (2.3) is sharp and bound (2.4) is sharp up to the logarithmic factor $\log |Q|$. This follows from the corresponding necessary conditions discussed in D.20. A lower bound.

D.12. In the case $q=\infty$ the discretization results are very different. We refer the reader to D.7 above and to the survey paper [33] for a discussion. The following result is from [33] (see Theorem 6.7 there). Let $\Lambda_{n}=\left\{k_{j}\right\}_{j=1}^{n}$ be a lacunary sequence: $k_{1}=1, k_{j+1} \geq b k_{j}, b>1, j=1, \ldots, n-1$. Assume that a finite set $\xi=\left\{\xi^{\nu}\right\}_{\nu=1}^{m} \subset \mathbb{T}$ has the following property

$$
\forall f \in \mathcal{T}\left(\Lambda_{n}\right) \quad\|f\|_{\infty} \leq K \max _{\nu}\left|f\left(\xi^{\nu}\right)\right| .
$$

Then

$$
m \geq(n / e) e^{C n / K^{2}}
$$

with a constant $C>0$ which may only depend on $b$.

Thus, for successful discretization of the uniform norm of general trigonometric polynomials we may need the number of points to be exponential in the dimension of the subspace. Namely, for good discretization of the uniform norm of functions from $\mathcal{T}\left(\Lambda_{n}\right)$ (see (2.5)) we need exponentially many points. It turns out that the exponential number of points is always enough for discretization of the uniform norm (see [72] and D.21 below). 


\subsection{General subspaces}

We begin with the definition of entropy numbers. Let $X$ be a Banach space and let $B_{X}$ denote the closed unit ball of $X$ with center at 0 . Denote by $B_{X}(y, r)$ a ball with center $y$ and radius $r: B_{X}(y, r):=\{x \in X:\|x-y\| \leq r\}$. For a compact set $A$ and a positive number $\varepsilon$ we define the covering number $N_{\varepsilon}(A)$ as follows

$$
N_{\varepsilon}(A):=N_{\varepsilon}(A, X):=\min \left\{n: \exists y^{1}, \ldots, y^{n}, y^{j} \in A: A \subseteq \cup_{j=1}^{n} B_{X}\left(y^{j}, \varepsilon\right)\right\} .
$$

It is convenient to consider the entropy numbers $\varepsilon_{k}(A, X)$ :

$$
\varepsilon_{k}(A, X):=\inf \left\{\varepsilon: \exists y^{1}, \ldots, y^{2^{k}} \in A: A \subseteq \cup_{j=1}^{2^{k}} B_{X}\left(y^{j}, \varepsilon\right)\right\} .
$$

In our definition of $N_{\varepsilon}(A)$ and $\varepsilon_{k}(A, X)$ we require $y^{j} \in A$. In a standard definition of $N_{\varepsilon}(A)$ and $\varepsilon_{k}(A, X)$ this restriction is not imposed. However, it is well known that these entropy number characteristics may differ at most by a factor 2. Namely, the following statement holds (see, for instance, [157], p.322, Theorem 7.1.1 and [153], p.208). Let $\varepsilon_{k}^{*}(A, X)$ be the entropy numbers without the restriction $y^{1}, \ldots, y^{2^{k}} \in A$. Then $\varepsilon_{k}^{*}(A, X) \leq \varepsilon_{k}(A, X) \leq 2 \varepsilon_{k}^{*}(A, X)$. As above, we use the following notation for the unit $L_{q}$-ball of $X_{N}$

$$
X_{N}^{q}:=\left\{f \in X_{N} \subset L_{q}:\|f\|_{q} \leq 1\right\} .
$$

Conditional results with entropy. We formulate here some results on sampling discretization, which are proved under conditions on the entropy numbers of the unit balls of finite-dimensional subspaces of interest. In a spirit, these results are connected with known results in learning theory (see, for instance, [153], Ch.4).

D.13. In the general case $q \in[1, \infty)$ the following statement holds (see [34]): There exists a positive constant $C(q)$ such that for any $N$-dimensional subspace $X_{N}$ satisfying the condition

$$
\varepsilon_{k}\left(X_{N}^{q}, L_{\infty}\right) \leq B(N / k)^{1 / q}, \quad 1 \leq k \leq N
$$

where $B \geq 1$, we have

$$
X_{N} \in \mathcal{M}(m, q, \varepsilon) \quad \text { provided } \quad m \geq C(q, \varepsilon) N B^{q}\left(\log _{2}(2 B N)\right)^{2} .
$$

Note that in the case $q=1, \varepsilon=1 / 2$, the above statement was proved in [155]. 
D.14. In the case $q \in[1, \infty)$ we have one more conditional result. In the above paragraph $\mathbf{D . 1 3}$ the condition is formulated in terms of the entropy numbers in the uniform norm $L_{\infty}$. Very recently, a new idea in this direction was developed in [86]. The corresponding theorem with the conditions imposed on the entropy numbers in a weaker metric than the uniform norm was proved in [86]. We now formulate that result. Let $Y_{s}:=\left\{y_{j}\right\}_{j=1}^{s} \subset \Omega$ be a set of sample points from the domain $\Omega$. Introduce a semi-norm

$$
\|f\|_{Y_{s}}:=\|f\|_{L_{\infty}\left(Y_{s}\right)}:=\max _{1 \leq j \leq s}\left|f\left(y_{j}\right)\right| .
$$

Clearly, for any $Y_{s}$ we have $\|f\|_{Y_{s}} \leq\|f\|_{\infty}$. The following result is from [86] (see Corollary 3.8 there): There exists a number $C_{1}(q)>0$ such that for $m$ and $B \geq 1$ satisfying

$m \geq C_{1}(q) N B^{q}(\log N)^{w(q)}, \quad w(1):=2, \quad w(q):=\max (q, 2)-1,1<q<\infty$, and for a subspace $X_{N}$ satisfying the condition: For any set $Y_{m} \subset \Omega$

$$
\varepsilon_{k}\left(X_{N}^{q}, L_{\infty}\left(Y_{m}\right)\right) \leq B(N / k)^{1 / q}, \quad 1 \leq k \leq N
$$

we have

$$
X_{N} \in \mathcal{M}(m, q), \quad 1 \leq q<\infty .
$$

The entropy assumption with the discretized uniform norm $\|f\|_{Y_{s}}$ was used to obtain upper bounds for the expectation of the supremum of a random process $\sum_{j=1}^{m} \varepsilon_{j}\left|f\left(y_{j}\right)\right|^{q}$, where $\varepsilon_{j}$ are i.i.d symmetric Bernoulli random variables. It would be interesting to understand whether one can relax the above entropy condition. For example, in [80] the following norm of a function was introduced

$$
\|f\|_{m, \infty}=\int_{\Omega} \ldots \int_{\Omega} \max _{1 \leq j \leq m}\left|f\left(y_{j}\right)\right| \mu\left(d y_{1}\right) \ldots \mu\left(d y_{m}\right) .
$$

This norm provided lower bounds for the expectation of the supremum of a random process in a similar setting (see also [47] and [48] for the development of ideas from [80]). One may wonder: Is it possible to replace the entropy condition (2.7) for each subset $Y_{s}$ with a single entropy condition similar to (2.7) with respect to norm (2.8) to get a good Marcinkiewicz-type discretization theorem? 
Conditional results with the Nikol'skii inequality. The following condition is widely used in discretization.

Condition E. The orthonormal system $\left\{u_{i}(x)\right\}_{i=1}^{N}$ defined on $\Omega$ satisfies Condition $\mathrm{E}$ with a constant $t$ if for all $x \in \Omega$

$$
\sum_{i=1}^{N}\left|u_{i}(x)\right|^{2} \leq N t^{2} .
$$

It is well known that Condition E is equivalent to the following Nikol'skii inequality (see, for instance, [96] for an explanation and [38] for a detailed discussion). We say that $X_{N}$ satisfies the Nikol'skii inequality for the pair $(2, \infty)$ if there exists a constant $t$ such that

$$
\|f\|_{\infty} \leq t N^{\frac{1}{2}}\|f\|_{2}, \quad \forall f \in X_{N} .
$$

It is known that (2.9) implies Condition $\mathrm{E}$ for any orthonormal basis of $X_{N}$ and that Condition $\mathrm{E}$ for an orthonormal basis of $X_{N}$ implies (2.9). Also, Condition E is closely related to the Christoffel function of the subspace $X_{N}$ (see D.26 below).

D.15. Consider the case $q=2$. In this case the sampling discretization problem for subspaces satisfying Condition $\mathrm{E}$ is solved in the sense of order (see [96]): There are three absolute positive constants $C_{i}, i=1,2,3$, such that for any $N$-dimensional subspace $X_{N} \subset L_{\infty}(\Omega)$ satisfying (2.9) we have

$$
X_{N} \in \mathcal{M}\left(m, 2, C_{1}, C_{2} t^{2}\right) \quad \text { provided } m \geq C_{3} t^{2} N .
$$

The above result has history beginning with the Rudelson's result from [129]. In the paper [129] it is formulated in terms of submatrices of an orthogonal matrix (see M.5 below). We reformulate it in our notation. Let $\Omega_{M}=\left\{x^{j}\right\}_{j=1}^{M}$ be a discrete set with the probability measure $\mu\left(x^{j}\right)=1 / M$, $j=1, \ldots, M$. Suppose that $X_{N} \subset L_{2}\left(\Omega_{M}, \mu\right)$ satisfies (2.9). Then for $\varepsilon \in(0,1)$ we have

$$
X_{N} \in \mathcal{M}(m, 2, \varepsilon) \quad \text { provided } \quad m \geq C \frac{t^{2}}{\varepsilon^{2}} N \log \frac{N t^{2}}{\varepsilon^{2}} .
$$

In the followup paper [130] more general results were obtained. In particular, these more general results imply $X_{N} \in \mathcal{M}(m, 2, \varepsilon)$ provided that $m \geq C(t / \varepsilon)^{2} N \log N$ for a general set $\Omega$ (see a discussion after Corollary 4.1 
below). In [155] it was demonstrated how the Bernstein-type concentration of measure inequalities for random matrices can be used for proving an ana$\log$ of the above Rudelson's result for a general $\Omega$. The proof in [155] is based on a different idea than Rudelson's proof.

Both (2.11) and its version for a general $\Omega$ provide sufficient conditions on $m$ for existence of a good set of cardinality $m$ for sampling discretization. These sufficient conditions are close to the necessary condition, which is $m \geq N$, but still have an extra $\log N$ factor in the bound for $m$. The result in (2.10) gives a sufficient condition on $m$ without an extra $\log N$ factor. The first result of that type was obtained in [154] where (2.10) was proved for special domains $\Omega_{M}$ and under a stronger condition than Condition E: $\sum_{i=1}^{N}\left|u_{i}\left(x^{j}\right)\right|^{2}=N, j=1, \ldots, M$.

D.16. Consider the case $q \in[1,2)$. The following result was obtained in [35]: Let $X_{N}$ be an $N$-dimensional subspace of $L_{\infty}(\Omega)$ satisfying (2.9) with $\log t \leq \alpha \log N$. Then we have

$$
X_{N} \in \mathcal{M}(m, q, \varepsilon) \quad \text { provided } \quad m \geq C(q, \alpha, \varepsilon) t N(\log N)^{3} .
$$

In the case $q \in(1,2)$ result $(2.12)$ was improved in [86] by replacing $(\log N)^{3}$ by $(\log N)^{2}$. It is interesting to note that in results for $q \in[1,2]$ the condition on $X_{N}$ - the Nikol'skii inequality for the pair $(2, \infty)$ - does not depend on $q$.

Let $p \in[1, \infty)$ and $X_{N} \subset L_{\infty}(\Omega)$. The inequality

$$
\|f\|_{\infty} \leq M\|f\|_{p}, \quad \forall f \in X_{N}
$$

is called the Nikol'skii inequality for the pair $(p, \infty)$ with the constant $M$. Denote

$$
M_{p}\left(X_{N}\right):=\sup _{f \in X_{N} ; f \neq 0}\|f\|_{\infty} /\|f\|_{p} .
$$

D.17. The understood case $q \in(2, \infty)$ is somewhat different from the case $q \in[1,2)$. In the former we use the Nikol'skii inequality for the pair $(q, \infty)$. The following results are known. It was proved in [86] that the Nikol'skii inequality for the pair $(q, \infty)$ with the constant $M_{q}\left(X_{N}\right)$ of the order $N^{1 / q}$ implies

$$
X_{N} \in \mathcal{M}(m, q) \text { provided } m \gg N(\log N)^{q} .
$$

It is pointed out in [159] that the Nikol'skii inequality for the pair $(q, \infty)$ with the constant $M_{q}\left(X_{N}\right)$ of the order $N^{1 / q}$ combined with the assumption that $X_{N} \in \mathcal{M}(s, \infty)$ with $s \leq a N^{c}$ imply

$$
X_{N} \in \mathcal{M}(m, q) \text { provided } m \gg N(\log N)^{3} .
$$


We also mention (see [86, Corollary 4.5]) that, for subspaces $X_{N}$ satisfying Condition E, one has

$$
X_{N} \in \mathcal{M}(m, q) \quad \text { provided } \quad m \gg C N^{\frac{q}{2}} \log N .
$$

For further results see Corollaries 4.2-4.4.

Comment. We discussed above conditional results for sampling discretization - D. 13-14 under the entropy condition and D. 15-17 under the Nikol'skii inequality condition. These two conditions are related. We will return to this connection at the end of Subsection 2.6.

Unconditional results. In the case of weighted discretization we can prove Marcinkiewicz-type discretization theorems for an arbitrary finite-dimensional subspace.

D.18. In the case $q=2$ we begin with he following result from [96]: There are three absolute positive constants $C_{i}, i=1,2,3$, such that for any (real or complex) $N$-dimensional subspace $X_{N} \subset L_{2}(\Omega, \mu)$ we have

$$
X_{N} \in \mathcal{M}^{w}\left(m, 2, C_{1}, C_{2}\right) \text { provided } m \geq C_{3} N .
$$

Moreover, the weights can be made positive.

The first result in that direction was obtained in [5] (see Theorem 3.1 there). In the case of a special domain $\Omega_{M}$ and real subspaces, the authors proved (2.13) with $C_{3}=b$, where $b>1$ is any number, $C_{1}=1$, and $C_{2}=\left(\frac{\sqrt{b}+1}{\sqrt{b}-1}\right)^{2}$. Further, it was observed in [33, Theorem 2.13] that this last result from [5] with a general probability space $(\Omega, \mu)$ in place of the discrete space $\left(\Omega_{M}, \mu\right)$ remains true (with other constant $\left.C_{2}\right)$ if $X_{N} \subset L_{4}(\Omega, \mu)$. It was proved in [35] (see Theorem 6.3 there) that the additional assumption $X_{N} \subset L_{4}(\Omega, \mu)$ can be dropped.

D.19. Consider the case $q \in[1,2)$. The following result was proved in [35]: For given $q \in[1,2)$ and $\varepsilon \in(0,1)$ there exists $C(q, \varepsilon)$ such that for any $N$-dimensional subspace of $L_{q}(\Omega, \mu)$ we have

$$
X_{N} \in \mathcal{M}^{w}(m, q, \varepsilon) \quad \text { provided } \quad m \geq C(q, \varepsilon) N(\log N)^{3} .
$$

D.20. An upper bound. We do not have results similar to paragraph D.19 in the case $q \in(2, \infty)$, but we have a corollary of $\mathbf{D . 1 8}$ for the case of even $q$. Let $q=2 s, s \in \mathbb{N}$. The case $s=1$ is covered by D.18. We consider the case $s \geq 2$. For a given $N$-dimensional subspace $X_{N} \subset L_{q}(\Omega, \mu)$ consider

$$
\left(X_{N}\right)^{s}:=\left\{f: f=f_{1} \times f_{2} \times \cdots \times f_{s}, f_{j} \in X_{N}, j=1, \ldots, s\right\} .
$$


It is well known and easy to see that $\left(X_{N}\right)^{s} \subset L_{2}(\Omega, \mu)$ and $\operatorname{dim}\left(X_{N}\right)^{s} \leq N^{s}$. For any $f \in X_{N}$ we have $f^{s} \in\left(X_{N}\right)^{s}$. Therefore, discretization of the $L_{2 s}$ norm of elements of $X_{N}$ follows from discretization of the $L_{2}$ norm of elements of $\left(X_{N}\right)^{s}$. Applying D.18 to $\left(X_{N}\right)^{s}$ we obtain the following result. There are three absolute positive constants $C_{i}, i=1,2,3$, such that for any (real or complex) $N$-dimensional subspace $X_{N} \subset L_{2 s}(\Omega, \mu)$ we have

$$
X_{N} \in \mathcal{M}^{w}\left(m, 2 s, C_{1}, C_{2}\right) \text { provided } m \geq C_{3} N^{s} .
$$

Moreover, the weights can be made positive.

Actually, in the case $2<q<\infty$ one has (see the discussion after Corollary 4.5 in MM.3)

$$
X_{N} \in \mathcal{M}^{w}(m, q, \varepsilon) \quad \text { provided } \quad m \geq C N^{\frac{q}{2}} \log N .
$$

D.20. A lower bound. We point out that, when the $L_{q}$ norm with $q \in(2, \infty)$ on a subspace $X_{N}$ is equivalent to the $L_{2}$ norm, it is necessary to have at least $c N^{q / 2}$ points for discretization with positive weights (see e.g. [8]). Indeed, assume that $\|f\|_{q} \leq M\|f\|_{2} \forall f \in X_{N}$ and for some points $\xi^{1}, \ldots, \xi^{m}$ and for some positive weights $\lambda_{1}, \ldots, \lambda_{m}$ one has

$$
c\|f\|_{q}^{q} \leq \sum_{\nu=1}^{m} \lambda_{\nu}\left|f\left(\xi^{\nu}\right)\right|^{q} \leq C\|f\|_{q}^{q}
$$

where $C>c>0$ are some fixed constants. Let $u_{1}, \ldots, u_{N}$ be an orthonormal basis in $X_{N}$. Then for each choice of signs $\varepsilon=\left(\varepsilon_{1}, \ldots, \varepsilon_{N}\right)$ for the function $f_{\varepsilon}:=\sum_{k=1}^{N} \varepsilon_{k} u_{k}$ one has

$$
c N^{q / 2}=c\left\|f_{\varepsilon}\right\|_{2}^{q} \leq c\left\|f_{\varepsilon}\right\|_{q}^{q} \leq \sum_{\nu=1}^{m} \lambda_{\nu}\left|f_{\varepsilon}\left(\xi^{\nu}\right)\right|^{q}=\sum_{\nu=1}^{m} \lambda_{\nu}\left|\sum_{k=1}^{N} \varepsilon_{k} u_{k}\left(\xi^{\nu}\right)\right|^{q} .
$$

We now assume that signs are chosen randomly and take the average:

$$
c N^{q / 2} \leq \sum_{\nu=1}^{m} \lambda_{\nu} \mathbb{E}_{\varepsilon}\left|\sum_{k=1}^{N} \varepsilon_{k} u_{k}\left(\xi^{\nu}\right)\right|^{q} \leq B_{q} \sum_{\nu=1}^{m} \lambda_{\nu}\left(\sum_{k=1}^{N}\left|u_{k}\left(\xi^{\nu}\right)\right|^{2}\right)^{q / 2},
$$

where we have applied the Khintchine inequality. We note that

$$
\lambda_{\nu}\left(\sum_{k=1}^{N}\left|u_{k}\left(\xi^{\nu}\right)\right|^{2}\right)^{q / 2}=\sup _{f \in X_{N},\|f\|_{2} \leq 1} \lambda_{\nu}\left|f\left(\xi^{\nu}\right)\right|^{q} \leq C M^{q}
$$


since

$$
\lambda_{\nu}\left|f\left(\xi^{\nu}\right)\right|^{q} \leq \sum_{\nu=1}^{m} \lambda_{\nu}\left|f\left(\xi^{\nu}\right)\right|^{q} \leq C\|f\|_{q}^{q} \leq C M^{q}\|f\|_{2}^{q} .
$$

Thus, $c N^{q / 2} \leq B_{q} C M^{q} m$ and $m \geq \frac{c}{C B_{q} M^{q}} N^{q / 2}$.

A classical example of a subspace with equivalent $L_{q}, 1 \leq q<\infty$, and $L_{2}$ norms is a subspace $\mathcal{T}\left(\Lambda_{n}\right)$, where $\Lambda_{n}=\left\{k_{j}\right\}_{j=1}^{n}$ is a lacunary sequence: $k_{1}=1, k_{j+1} \geq b k_{j}, b>1, j=1, \ldots, n-1$.

As another example of a space with equivalent $L_{q}$ and $L_{2}$ norms, consider the space $X_{N}:=\left\{\langle\cdot, \mathbf{y}\rangle: \mathbf{y} \in \mathbb{R}^{N}\right\} \subset L^{q}\left(Q_{N}\right)$, where $Q_{N}:=\left[-\frac{1}{2}, \frac{1}{2}\right]^{N}-$ is the unit cube endowed with the standard Lebesgue measure. It is known (see Theorem 2.4.6 in [25]) that for each $q$ there is a constant $c_{q}$, dependent only on $q$ and, in particular, independent of the dimension $N$, such that $\|f\|_{q} \leq c_{q}\|f\|_{2} \forall f \in X_{N}$. Moreover, $X_{N}$ satisfies the $(2, \infty)$ Nikol'skii-type inequality assumption with constant 3 i.e. $\|f\|_{\infty} \leq 3 N^{1 / 2}\|f\|_{2} \forall f \in X_{N}$.

D.21. The recent paper [72] addresses sampling discretization of the uniform norm. In particular, it is proved there that for discretization of the uniform norm of elements of any $N$-dimensional subspace of $L_{\infty}(\Omega)=\mathcal{C}(\Omega)$ it is sufficient to use $e^{C N}$ sample points. The following statement is proved in [72]. Let $X_{N}$ be an $N$-dimensional subspace of $L_{\infty}(\Omega)$. There exists a set $\xi=\left\{\xi^{\nu}\right\}_{\nu=1}^{m}$ of $m \leq 9^{N}$ points such that for any $f \in X_{N}$ we have

$$
\|f\|_{\infty} \leq 2 \max _{\nu}\left|f\left(\xi^{\nu}\right)\right| \quad \text { which means } \quad X_{N} \in \mathcal{M}\left(9^{N}, \infty, 1 / 2\right) .
$$

We now mention some results, where, in contrast to our setting, the constant $C_{1}$ in the Bernstein discretization problem is allowed to depend on $N$. The following result was obtained in [112] (see Proposition 1.2.3 there). Let $X_{N}$ be an $N$-dimensional subspace of $\mathcal{C}(\Omega)$ and let $\varepsilon>0$. There exists a set $\xi=\left\{\xi^{\nu}\right\}_{\nu=1}^{N}$ of $N$ points such that for any $f \in X_{N}$ we have

$$
\|f\|_{\infty} \leq(N+\varepsilon) \max _{\nu}\left|f\left(\xi^{\nu}\right)\right| \text {. }
$$

The following conditional result, which connects the upper bound in the discretization theorem for the uniform norm with the Nikol'skii-type inequality between $\mathcal{C}$ and $L_{2}$ norms, was proved in [33]. Let $\Omega:=[0,1]^{d}$. Assume that a real $N$-dimensional subspace $Y_{N} \subset \mathcal{C}(\Omega)$ satisfies the Nikol'skii-type inequality: For any $f \in Y_{N}$

$$
\|f\|_{\infty} \leq H(N)\|f\|_{2}, \quad\|f\|_{2}:=\left(\int_{\Omega}|f(\mathbf{x})|^{2} d \mu\right)^{1 / 2}
$$


where $\mu$ is the Lebesgue measure on $\Omega$. Then for any $a>1$ there exists a set $\xi(m)=\left\{\xi^{j}\right\}_{j=1}^{m} \subset \Omega$ with the property: $m \leq a N$ and for any $f \in Y_{N}$ we have

$$
\|f\|_{\infty} \leq C(a) H(N) \max _{1 \leq j \leq m}\left|f\left(\xi^{j}\right)\right|,
$$

where $C(a)$ is a positive constant.

\subsection{Real algebraic polynomials}

In this subsection we discuss the case $\Omega=[-1,1]^{d}$ with a probability measure $\mu$ such that $d \mu=w(\mathbf{x}) d \mathbf{x}$. A subspace $X_{N}$ will be a subspace of algebraic polynomials.

D.22. We begin with direct corollaries of results on the trigonometric polynomials. For $\mathbf{x} \in[-1,1]^{d}$ and $\mathbf{t} \in[0, \pi]^{d}$ consider the following change of variables $x_{j}=-\cos t_{j}, j=1, \ldots, d$. Also, consider the measure $\mu_{c}$ with density $w_{c}(\mathbf{x}):=\pi^{-d} \prod_{j=1}^{d}\left(1-x_{j}^{2}\right)^{-1 / 2}$ - the Chebyshev measure - on $[-1,1]^{d}$. Then we have

$$
\int_{[-1,1]^{d}}|f(\mathbf{x})|^{q} w_{c}(\mathbf{x}) d \mathbf{x}=\pi^{-d} \int_{[0, \pi]^{d}}\left|f\left(-\cos t_{1}, \ldots,-\cos t_{d}\right)\right|^{q} d \mathbf{t} .
$$

Thus, result D.3 implies the following relation. Let $\mathbf{N}=\left(N_{1}, \ldots, N_{d}\right)$ and $\mathcal{P}(\mathbf{N}, d)$ be the subspace of algebraic polynomials of degree $N_{j}$ in the variable $j, j=1, \ldots, d$. Then we have for $1 \leq q<\infty$

$$
\mathcal{P}(\mathbf{N}, d) \in \mathcal{M}\left(C(d) \operatorname{dim} \mathcal{P}(\mathbf{N}, d), L_{q}\left([-1,1]^{d}, \mu_{c}\right)\right), \quad d \mu_{c}=w_{c}(\mathbf{x}) d \mathbf{x},
$$

and

$$
\mathcal{P}(\mathbf{N}, d) \in \mathcal{M}\left(C(d) \operatorname{dim} \mathcal{P}(\mathbf{N}, d), L_{\infty}\left([-1,1]^{d}\right)\right) .
$$

D.23. Consider special algebraic polynomials - Chebyshev polynomials

$$
T_{k}(x):=\frac{1}{2}\left(\left(x+\left(x^{2}-1\right)^{1 / 2}\right)^{k}+\left(x-\left(x^{2}-1\right)^{1 / 2}\right)^{k}\right), \quad k=0,1, \ldots
$$

The system $\left\{T_{k}(x)\right\}_{k=0}^{\infty}$ forms an orthonormal basis in $L_{2}\left([-1,1], \mu_{c}\right)$. Note that $T_{k}(-\cos t)=(-1)^{k} \cos k t$. Denote

$$
T_{\mathbf{k}}(\mathbf{x}):=\prod_{j=1}^{d} T_{k_{j}}\left(x_{j}\right), \quad \mathbf{k}=\left(k_{1}, \ldots, k_{d}\right), \quad \mathbf{x}=\left(x_{1}, \ldots, x_{d}\right) .
$$


For a finite set $Q \subset \mathbb{Z}_{+}^{d}$ denote

$$
\mathcal{C}(Q, d):=\left\{f: f(\mathbf{x})=\sum_{\mathbf{k} \in Q} c_{\mathbf{k}} T_{\mathbf{k}}(\mathbf{x})\right\} .
$$

Then, result D.15 implies for any $Q \subset \mathbb{Z}_{+}^{d}$

$$
\mathcal{C}(Q, d) \in \mathcal{M}\left(m, L_{2}\left([-1,1]^{d}, \mu_{c}\right), C_{1}, C_{2}\right) \text { provided } m \geq C_{3}|Q| .
$$

We now consider sets $Q \subset \mathbb{Z}_{+}^{d}$ with the property: if $\mathbf{k}=\left(k_{1}, \ldots, k_{d}\right) \in Q$, then the box $\prod_{j=1}^{d}\left[0, k_{j}\right]$ also belongs to $Q$. These sets were used in the multivariate approximation of periodic functions in [151] (see also [157], p.141). There, these sets were identified by saying: " $Q$ has the property $S$ ". Later, sets with this property have become important in Stochastic PDEs, where these sets are called lower sets.

For a finite set $Q \subset \mathbb{Z}_{+}^{d}$ denote

$$
\mathcal{P}(Q, d):=\left\{f: f(\mathbf{x})=\sum_{\mathbf{k} \in Q} c_{\mathbf{k}} \mathbf{x}^{\mathbf{k}}\right\}, \quad \mathbf{x}^{\mathbf{k}}=\prod_{j=1}^{d} x_{j}^{k_{j}} .
$$

Note that for any lower set $Q$ we have $\mathcal{P}(Q, d)=\mathcal{C}(Q, d)$. Therefore, $(2.14)$ implies: For any lower set $Q$ we have

$$
\mathcal{P}(Q, d) \in \mathcal{M}\left(m, L_{2}\left([-1,1]^{d}, \mu_{c}\right), C_{1}, C_{2}\right) \text { provided } m \geq C_{3}|Q| .
$$

D.24. We formulate a result from [63]. Consider the case $d=1$ and the measure $\mu(\gamma)$ with density $w(x, \gamma)=\left(1-x^{2}\right)^{\gamma-1 / 2},-1 / 2<\gamma<\infty$. Then we have

$$
\mathcal{P}(n-1,1) \in \mathcal{M}^{w}\left(n, L_{q}([-1,1], \mu(\gamma)), \quad 1<q<\infty .\right.
$$

Note that in the case $\gamma=1 / 2$ the measure $\mu(1 / 2)$ is the standard Lebesgue measure. The reader can find some discretization results for this case in the book [137]. In particular, it is known (see [29] and [136]) that if we restrict ourselves to uniformly distributed points $\xi=\left\{\xi^{j}\right\}_{j=0}^{2 m}, \xi^{j}=(j-m) / m$, then for sampling discretization of the $L_{q}([-1,1], \mu(1 / 2))$ norm of polynomials from $\mathcal{P}(n, 1)$ it is necessary and sufficient that $m$ grows as $n^{2}$ with $n \rightarrow \infty$.

D.25. There are some recent papers on discretization in the uniform norm $L_{\infty}$ of subspaces of algebraic polynomials defined on a set $\Omega \subset \mathbb{R}^{d}$. 
Denote by $\mathcal{P}_{n}^{d}$ the set of real algebraic polynomials of total degree $\leq n$. In other words, $\mathcal{P}_{n}^{d}$ is the set of real polynomials from $\mathcal{P}\left(\Delta_{n}, d\right)$, where $\Delta_{n}:=\left\{\mathbf{k} \in \mathbb{Z}_{+}^{d}:\|\mathbf{k}\|_{1}:=k_{1}+\cdots+k_{d} \leq n\right\}$, the cardinality $\left|\mathcal{P}_{n}^{d}\right|=\left(\begin{array}{c}n+d-1 \\ d-1\end{array}\right)$, $\left|\mathcal{P}_{n}^{d}\right|^{\frac{1}{d-1}} \asymp \frac{n+d-1}{d-1}$. The following result was obtained in [90]: Let $d=2$ and let $\Omega \subset \mathbb{R}^{2}$ be a convex compact set with nonempty interior, then for $\varepsilon \in(0,1)$

$$
\mathcal{P}_{n}^{2} \in \mathcal{M}\left(4 \cdot 10^{5}(n / \varepsilon)^{2}, L_{\infty}(\Omega),(1+\varepsilon)^{-1}\right)
$$

The following result is from [11]: Let $\Omega \subset \mathbb{R}^{d}, d \in \mathbb{N}$, be a compact set with nonempty interior, then there exist positive constants $C_{0}(d)$ and $C_{1}(d)$ such that for any $m \geq C_{0}(d)(n \log n)^{d}$ we have

$$
\mathcal{P}_{n}^{d} \in \mathcal{M}\left(m, L_{\infty}(\Omega), C_{1}(d)\right) .
$$

D.26. We continue a discussion from D.25 and present a conditional result from [126]. Let $\left\{P_{j}\right\}_{j=1}^{N}$ be an orthonormal basis of $\mathcal{P}_{n}^{d}$ with respect to the normalized Lebesgue measure on $\Omega$. Define the Christoffel function for the $\mathcal{P}_{n}^{d}$ as

$$
\lambda_{n}(\mathbf{x}, \Omega):=\left(\sum_{j=1}^{N} P_{j}(\mathbf{x})^{2}\right)^{-1 / 2} .
$$

The following conditional result is proved in [126] with the aid of a lemma from [14]: Suppose $\Omega$ is such that

$$
\lambda_{n}(\mathbf{x}, \Omega) \leq C(\Omega) \lambda_{2 n}(\mathbf{x}, \Omega), \quad \mathbf{x} \in \Omega
$$

Then

$$
\mathcal{P}_{n}^{d} \in \mathcal{M}\left(C(\Omega) \operatorname{dim}\left(\mathcal{P}_{n}^{d}\right), \infty\right)
$$

D.27. We now formulate one recent result for the case $1 \leq q \leq \infty$. The following result was obtained in [32]: Let $\Omega$ be a bounded $C^{2}$-domain in $\mathbb{R}^{d}$ with the normalized Lebesgue measure $\mu_{L}$ on it. Then for $1 \leq q<\infty$

$$
\mathcal{P}_{n}^{d} \in \mathcal{M}^{w}\left(C(\Omega) \operatorname{dim}\left(\mathcal{P}_{n}^{d}\right), L_{q}\left(\Omega, \mu_{L}\right), 1 / 2,3 / 2\right)
$$

and for $q=\infty$

$$
\mathcal{P}_{n}^{d} \in \mathcal{M}\left(C(\Omega) \operatorname{dim}\left(\mathcal{P}_{n}^{d}\right), L_{\infty}(\Omega)\right) .
$$

A comment on other settings. The above discussion in this section shows that there is only one case, namely the case $q=2$, where the weighted 
sampling discretization problem is solved (in the sense of order). This result is presented in D.18. We point out that there are many ingredients in the setting of the sampling discretization problem. Here they are: $(1) L_{q}(\Omega, \mu)$, which contains three ingredients: $1 \leq q \leq \infty, \Omega$, and the measure $\mu$; (2) the subspace $X_{N}$; (3) restrictions on the weights $\lambda_{1}, \ldots, \lambda_{m}$ in the discretization formula (2.2). Clearly, a result like D.18 with $1 \leq q<\infty$ and equal weights will solve the problem of discretization for all $1 \leq q<\infty$. Note, that results of $\mathbf{D} .7$ show that there is no analog of D.18 for $q=\infty$. However, there is no known result at the level of generality of $\mathbf{D . 1 8}$ for $1 \leq q<\infty$ distinct from 2. Therefore, in order to obtain results on discretization we impose some restrictions on the above mentioned ingredients of the problem. Typical restrictions are as follows. Instead of a general subspace $X_{N}$ we study a specific one, say, trigonometric or algebraic polynomials. Instead of a general domain $\Omega$ we study a specific one, say, a cube or a convex domain. Instead of a general measure $\mu$ we study a specific one, say, the Lebesgue or the Chebyshev measure. We now comment on a special case, when $X_{N}$ is the $\mathcal{P}_{n}^{d}$. We discuss different sets $\Omega$. Let us begin with the case $d=2$. Consider a unit circle $S^{1}$ as the $\Omega$. Then it is clear that the subspace $\mathcal{P}_{n}^{2}$ restricted to $S^{1}$ becomes the subspace of the trigonometric polynomials (with the angle from the polar coordinates as the argument) of degree $n$. There is a number of papers, where $\mathcal{P}_{n}^{d}$ is restricted to the sphere $S^{d-1}$ or to a part of it. We briefly mention here some of these papers and refer the reader to these papers for further discussion. The authors of [100] proved the weighted Marcinkiewicz discretization theorems with general doubling measures for algebraic polynomials on intervals and for trigonometric polynomials on circles, with the number of required sample points comparable to the dimension of the corresponding polynomial spaces. The paper [31] extends the results of [100] to the sphere and the ball. Weighted Marcinkiewicz discretization theorems with doubling measures were established in this paper for spherical polynomials on the unit sphere and for algebraic polynomials on the unit ball. Further, the weighted Marcinkiewicz discretization theorems for spherical polynomials on spherical caps were established in [36]. In both [31] and [36], the number of sampling points required in the Marcinkiewicz discretization theorems is asymptotically equivalent to the dimension of the corresponding polynomial spaces. 


\subsection{Complex algebraic polynomials}

The discretization theorems of Bernstein and Marcinkiewicz-Zygmund (see D.2 and D.3) were obtained as natural corollaries of the Bernstein and Zygmund inequalities for derivatives of the trigonometric polynomials. The corresponding inequalities for derivatives have been established and used in the proofs of the inverse theorems of approximation - i.e. statements on smoothness properties of a function, which is approximated well by trigonometric polynomials. Applications of these inequalities to discretization problems appeared two decades later, despite the fact that an approach to construction of economical nets, which provide good sampling discretization of a function, based on information of its derivative, is absolutely natural. We demonstrate this remark on the example of a subspace $X_{N} \subset \mathcal{C}(\Omega), \operatorname{dim} X_{N}=N$, consisting of smooth functions defined on a compact subset $\Omega \subset \mathbb{R}^{d}$. Let $B_{N}:=X_{N}^{\infty}$ be the unit $L_{\infty}$-ball in $X_{N}$. Suppose that for the gradient $\nabla f$ we have the following bound for each $f \in B_{N}$

$$
|\nabla f(\mathbf{x})| \leq M, \quad \mathbf{x} \in \Omega
$$

where, as usual, $|\mathbf{y}|=\|\mathbf{y}\|_{\ell_{2}^{d}}=\left(\left|y_{1}\right|^{2}+\ldots+\left|y_{d}\right|^{2}\right)^{1 / 2}$ for $\mathbf{y}=\left(y_{1}, \ldots, y_{d}\right) \in \mathbb{R}^{d}$. Then for any finite subset $\Lambda \subset \Omega$, which forms an $(2 M)^{-1}$-net for the $\Omega$ in the metric $\ell_{2}^{d}$, and for each $f \in B_{N}$ the following inequality holds

$$
\max _{\xi \in \Lambda}|f(\xi)| \leq\|f\|_{\mathcal{C}(\Omega)} \leq 2 \max _{\xi \in \Lambda}|f(\xi)|
$$

Indeed, let $\mathbf{z} \in \Omega$ be one of the points, where the norm of $f \in B_{N}$ is achieved, say, $|f(\mathbf{z})|=\|f\|_{\mathcal{C}(\Omega)}=1$. Then for $\xi \in \Lambda$ such that $|\mathbf{z}-\xi| \leq(2 M)^{-1}$ we obtain

$$
|f(\xi)| \geq|f(\mathbf{z})|-|f(\mathbf{z})-f(\xi)| \geq 1-|\mathbf{z}-\xi|\|\nabla f\|_{\mathcal{C}(\Omega)} \geq 1 / 2 .
$$

Typically, establishing discretization results for subspaces of smooth functions (first of all for subspaces of polynomials) is reduced to the proof of the corresponding inequalities of the Bernstein-type.

D.28. We begin with results from [68] and its unpublished followup note [58] on discretization in the subspace $\Pi(d, N)$ of homogeneous polynomials on $d$ complex variables of degree $N$ restricted onto the complex sphere

$$
\mathcal{S}^{d}:=\left\{\mathbf{z}=\left(z_{1}, \ldots, z_{d}\right) \in \mathbb{C}^{d}:|\mathbf{z}|=\left(\left|z_{1}\right|^{2}+\cdots+\left|z_{d}\right|^{2}\right)^{1 / 2}=1\right\} .
$$


It is proved in [68] that the absolute value of the tangential derivative of a function $|P|, P \in \Pi(d, N)$, is bounded by $C N^{1 / 2}\|P\|_{\mathcal{C}\left(\mathcal{S}^{d}\right)}$ and, therefore, for any two $\left|\mathbf{z}^{1}\right|=\left|\mathbf{z}^{2}\right|=1$

$$
|| P\left(\mathbf{z}^{1}\right)|-| P\left(\mathbf{z}^{2}\right)|| \leq C N^{1 / 2} d\left(\mathbf{z}^{1}, \mathbf{z}^{2}\right)\|P\|_{\mathcal{C}\left(\mathcal{S}^{d}\right)}
$$

where $d\left(\mathbf{z}^{1}, \mathbf{z}^{2}\right)$ is the spherical distance between $\mathbf{z}^{1}$ and $\mathbf{z}^{2}$, and $C$ is an absolute constant. By the argument explained above (2.15) implies (see [68]):

$$
\Pi(d, N) \in \mathcal{M}\left(C_{d} N^{d-1}, \infty, 1 / 2\right) .
$$

Note that $\operatorname{dim} \Pi(d, N) \asymp N^{d-1}$. Therefore, relation (2.16) shows that alike subspaces of trigonometric polynomials subspaces $\Pi(d, N)$ allow good discretization with the number of sampling points of the same order as their dimensions. In the case of the uniform norm, the corresponding $\varepsilon$-nets of $\Omega$ with $\varepsilon \asymp N^{-1 / 2}$ can be taken as those point sets. Relation (2.16) was successfully used to construct uniformly bounded bases in the spaces of holomorphic functions in the $d$-dimensional ball $\mathcal{B}^{d}$. Also, it was used to prove the existence of nontrivial inner functions in $\mathcal{B}^{d}$ (see [18], [15], and [68]). Note that L. Hörmander, [58], found the best constant in the inequality (2.15):

$$
C_{\text {min }}=(1-1 / N)^{(1-N) / 2} \leq e^{1 / 2}, \quad N=1,2, \ldots
$$

Also, a class of surfaces for which an analog of (2.16) holds was suggested in [58].

D.29. An analog of (2.16) for the $L_{p}$ norms, $1 \leq p \leq \infty$, was obtained in [173]. Namely, it was proved in [173] that for $1 \leq p \leq \infty$

$$
\Pi(d, N) \in \mathcal{M}\left(C_{d} \operatorname{dim}(\Pi(d, N)), p, C_{1}(d), C_{2}(d)\right) .
$$

The maximal $c_{d} N^{-1 / 2}$-distinguishable subset $\Lambda \subset \Omega$ can be taken as a good discretization point set.

D.30. In the case of functions on real variables, i.e. in the case of subspaces $\mathcal{P}_{n}^{d}$ restricted to the unit sphere in $\mathbb{R}^{d}$, analogs of (2.17) were considered later in [101]. In this case a good discretization point set was constructed in a different way. The unit sphere is divided into disjoint subsets of equal measure and of diameters $\leq c_{d} N^{-1}$. Then, a good discretization point set is formed by including one point from each subset of the subdivision. An interesting application of the Marcinkiewicz-Zygmund inequalities from [101] (the case $p=1$ ) was found in [12]. The authors of [12] solved the 
problem of existence of a cubature formula with equal weights defined on the unit sphere of $\mathbb{R}^{d}$ with the minimal in the sense of order number of points $\left(\asymp N^{d-1}\right.$ ), which is exact for algebraic polynomials of total degree $\leq N$ (see [13] for further developments).

D.31. We now briefly comment on some results on discretization on manifolds (see [42], [122], [26] and references therein). Let $M$ be a compact Riemannian manifold. Consider a negative definite self adjoint elliptic differential operator of the second order defined on $L_{2}(M)$. Optimal in the sense of order results on sampling discretization of the $L_{p}$ norm, $1 \leq p \leq \infty$, of elements of subspaces generated by eigenfunctions corresponding to the eigenvalues of the operator not exceeding a given number $N$ are obtained in [122]. Similar results on sampling discretization were also established on compact two-point homogeneous spaces in the paper [26].

D.32. The author of [138] found sufficient conditions on a generating function $\varphi$, which guarantee that the Marcinkiewicz-Zygmund inequalities hold for subspaces spanned by the corresponding system of periodic wavelets for all $L_{p}, 1 \leq p \leq \infty$.

It is clear that many of these results in Section 2 can be formulated as results on the quasi-matrix property (see Definition 2.1 above) of the corresponding system. We note that the concept of quasi-matrix system was used in [74] for establishing general lower bounds for the best $m$-term approximation in the $L_{1}$ norm.

\subsection{Sampling recovery}

The problem of recovery (reconstruction) of an unknown function defined on a subset of $\mathbb{R}^{d}$ from its samples at a finite number of points is a fundamental problem of pure and applied mathematics. The goal is to construct recovering operators (algorithms) which are good in the sense of accuracy, stability, and computational complexity. In this subsection we discuss the issue of accuracy. It turns out that, in the case of recovery in the $L_{2}$ norm, the weighted least squares algorithms are reasonably good recovering methods. The corresponding analysis is based on recent deep results in discretization of the $L_{2}$ norms of functions from finite-dimensional subspaces (see D.18 above).

Recall the setting of optimal recovery. For a fixed $m$ and a set of points $\xi:=\left\{\xi^{j}\right\}_{j=1}^{m} \subset \Omega$, let $\Phi$ be a linear operator from $\mathbb{C}^{m}$ into $L_{p}(\Omega, \mu)$. Denote 
for a class $\mathbf{F}$

$$
\varrho_{m}\left(\mathbf{F}, L_{p}\right):=\inf _{\operatorname{linear} \Phi ; \xi} \sup _{f \in \mathbf{F}}\left\|f-\Phi\left(f\left(\xi^{1}\right), \ldots, f\left(\xi^{m}\right)\right)\right\|_{p} .
$$

This recovery procedure is linear; the following modification of it is also of interest. We allow any mapping $\Phi: \mathbb{C}^{m} \rightarrow X_{N} \subset L_{p}(\Omega, \mu)$ where $X_{N}$ is a linear subspace of dimension $N \leq m$ and define

$$
\varrho_{m}^{*}\left(\mathbf{F}, L_{p}\right):=\inf _{\Phi ; \xi ; X_{N}, N \leq m} \sup _{f \in \mathbf{F}}\left\|f-\Phi\left(f\left(\xi^{1}\right), \ldots, f\left(\xi^{m}\right)\right)\right\|_{p} .
$$

In both of the above cases we build an approximant, which comes from a linear subspace of dimension at most $m$. It is natural to compare quantities $\varrho_{m}\left(\mathbf{F}, L_{p}\right)$ and $\varrho_{m}^{*}\left(\mathbf{F}, L_{p}\right)$ with Kolmogorov widths. Let $\mathbf{F} \subset L_{p}$ be a centrally symmetric compact. The quantities

$$
d_{n}\left(\mathbf{F}, L_{p}\right):=\inf _{\left\{u_{i}\right\}_{i=1}^{n} \subset L_{p}} \sup _{f \in \mathbf{F}} \inf _{c_{i}}\left\|f-\sum_{i=1}^{n} c_{i} u_{i}\right\|_{p}, \quad n=1,2, \ldots,
$$

are called the Kolmogorov widths of $\mathbf{F}$ in $L_{p}$. In the definition of Kolmogorov widths we take for $f \in \mathbf{F}$ an approximating element from $U:=\operatorname{span}\left\{u_{i}\right\}_{i=1}^{n}$ the element of best approximation. This means that in general (i.e. if $p \neq 2$ ) this method of approximation is not linear.

We have the following obvious inequalities

$$
d_{m}\left(\mathbf{F}, L_{p}\right) \leq \varrho_{m}^{*}\left(\mathbf{F}, L_{p}\right) \leq \varrho_{m}\left(\mathbf{F}, L_{p}\right)
$$

The characteristics $\varrho_{m}$ and $\varrho_{m}^{*}$ are well studied for many particular classes of functions. For an exposition of known results we refer to the books [164], [112], [39], [157], [113]-[115] and references therein. The characteristics $\varrho_{m}^{*}$ and $\varrho_{m}$ are inspired by the concepts of the Kolmogorov width and the linear width. Probably, $\varrho_{m}^{*}$ were introduced in [164] and $\varrho_{m}$ in [152].

In $\mathbf{R} .1$ and $\mathbf{R . 2}$ we consider the case $p=2$, i.e. recovery takes place in the Hilbert space $L_{2}$.

R.1. The following general inequality was proved in [161] with the aid of result D.18: There exist two positive absolute constants $b$ and $B$ such that for any compact subset $\Omega$ of $\mathbb{R}^{d}$, any probability measure $\mu$ on it, and any compact subset $\mathbf{F}$ of $\mathcal{C}(\Omega)$ we have

$$
\varrho_{b n}\left(\mathbf{F}, L_{2}(\Omega, \mu)\right) \leq B d_{n}\left(\mathbf{F}, L_{\infty}\right) .
$$


Note, that it is pointed out in [96] (see Theorem 3.4 of v2) that in the case of real spaces the constant $b>1$ can be taken arbitrarily close to 1 and in the case of complex spaces - the constant $b>2$ arbitrarily close to 2 with the constant $B$ allowed to depend on $b$. We refer the reader to the very recent paper [28] (also see references therein) for a discussion of the $L_{2}$ recovery when sampling points are drawn according to some random distribution.

R.2. Probably, the first paper, which gave a general upper bound on the sampling numbers $\varrho_{n}\left(\mathbf{F}, L_{2}\right)$ in terms of the Kolmogorov numbers $d_{k}\left(\mathbf{F}, L_{2}\right)$ is [172]. Recently an outstanding progress has been done in the sampling recovery in the $L_{2}$ norm (see, for instance, [27], [88], [89], [110], [28], [162], and [82], [83]). We give a very brief comments on those interesting results. For special sets $\mathbf{F}$ (in the reproducing kernel Hilbert space setting) the following inequality is known (see [110] and [88]):

$$
\varrho_{n}\left(\mathbf{F}, L_{2}\right) \leq C\left(\frac{\log n}{n} \sum_{k \geq c n} d_{k}\left(\mathbf{F}, L_{2}\right)^{2}\right)^{1 / 2}
$$

with absolute constants $C, c>0$. We refer the reader for further results in this direction to the paper [89]. In particular, the close to optimal bounds on $\varrho_{n}\left(\mathbf{F}, L_{2}\right)$ in terms of $d_{k}\left(\mathbf{F}, L_{2}\right)$ are obtained there for general sets $\mathbf{F}$.

The above results were proved with the aid of a classical type of algorithm - weighted least squares. A variant of this method - the empirical risk minimization - is a standard tool in learning theory and nonparametric statistics (see, for instance, [54] and [153], Ch.4). The weighted least squares algorithm was successfully applied in the sampling recovery for the first time in $[27]$.

Let $X_{N}$ be an $N$-dimensional subspace of the space of continuous functions $\mathcal{C}(\Omega)$ and let $\mathbf{w}:=\left(w_{1}, \ldots, w_{m}\right) \in \mathbb{R}^{m}$ be a positive weight, i.e. $w_{i}>0$, $i=1, \ldots, m$. Consider the following classical weighted least squares recovery operator (algorithm) (see, for instance, [27])

$$
\ell 2 \mathbf{w}\left(\xi, X_{N}\right)(f):=\arg \min _{u \in X_{N}}\|S(f-u, \xi)\|_{2, \mathbf{w}}, \quad \xi=\left\{\xi^{j}\right\}_{j=1}^{m} \subset \Omega,
$$

where

$$
\|S(g, \xi)\|_{2, \mathbf{w}}:=\left(\sum_{\nu=1}^{m} w_{\nu}\left|g\left(\xi^{\nu}\right)\right|^{2}\right)^{1 / 2} .
$$

The above weighted least square operator is a linear operator. This linearity guarantees that its application gives an upper bound for $\varrho_{n}\left(\mathbf{F}, L_{2}\right)$. 
Along with the operator $\ell 2 \mathbf{w}\left(\xi, X_{N}\right)(f)$ we can also consider the operator $\ell p \mathbf{w}\left(\xi, X_{N}\right)(f), 1 \leq p<\infty$, and the operator $\ell \infty\left(\xi, X_{N}\right)(f)$. These operators are not linear operators for $p \neq 2$. Therefore, their applications provide upper bounds for $\varrho_{n}^{*}\left(\mathbf{F}, L_{p}\right)$ but not for $\varrho_{n}\left(\mathbf{F}, L_{p}\right)$.

R.3. Inequality (2.18) gives a bound on sample recovery in $L_{2}$ with the right hand side being a characteristic of the set $\mathbf{F}$ measured in the uniform norm. We now discuss an analog of (2.18) for the $L_{p}$ spaces with $p \in(2, \infty)$. If instead of D.18 we use D.20. An upper bound, then the proof from [161] allows us to establish an analog of (2.18). Then similarly to R.1 we obtain the following statement. Let $p=2 s, s \in \mathbb{N}$. There exist two positive absolute constants $b$ and $B$ such that for any compact subset $\Omega$ of $\mathbb{R}^{d}$, any probability measure $\mu$ on it, and any compact subset $\mathbf{F}$ of $\mathcal{C}(\Omega)$ we have

$$
\varrho_{b n^{p / 2}}^{*}\left(\mathbf{F}, L_{p}(\Omega, \mu)\right) \leq B d_{n}\left(\mathbf{F}, L_{\infty}\right) .
$$

R.4. In the case $p=\infty$ we use the proof from [161] with $\mathbf{D . 1 8}$ replaced by D.21. Then similarly to R.1, R.3 we obtain the following statement. There exists a positive absolute constant $B$ such that for any compact subset $\Omega$ of $\mathbb{R}^{d}$ and any compact subset $\mathbf{F}$ of $\mathcal{C}(\Omega)$ we have

$$
\varrho_{9^{n}}^{*}\left(\mathbf{F}, L_{\infty}(\Omega)\right) \leq B d_{n}\left(\mathbf{F}, L_{\infty}\right)
$$

It is interesting to compare the above inequality (2.20) with the following result of E. Novak (see [112], Proposition 1.2.5)

$$
\varrho_{n}^{*}\left(\mathbf{F}, L_{\infty}(\Omega)\right) \leq(n+1) d_{n}\left(\mathbf{F}, L_{\infty}\right) .
$$

Inequality (2.20) complements inequality (2.21) in the case, when the sequence $\left\{d_{n}\left(\mathbf{F}, L_{\infty}\right)\right\}$ decays slowly, slower than $1 / n$.

We refer the reader for some recent results on sampling recovery in the uniform norm to the paper [125].

Very recently the result R.1 was applied to estimate the error of optimal sampling recovery in $L_{2}$ of functions with small mixed smoothness (see [162]). In the case of small smoothness we cannot use R.2 because the series in (2.19) diverges. This calls to mind an interesting phenomenon, which was discovered in [162]. We illustrate that phenomenon on the example of the $\mathbf{H}_{p}^{r}$ classes. Let $\mathbf{t}=\left(t_{1}, \ldots, t_{d}\right)$ and $\Delta_{\mathbf{t}}^{l} f(\mathbf{x})$ be the mixed $l$-th difference with step $t_{j}$ in the variable $x_{j}$, that is

$$
\Delta_{\mathbf{t}}^{l} f(\mathbf{x}):=\Delta_{t_{d}, d}^{l} \ldots \Delta_{t_{1}, 1}^{l} f\left(x_{1}, \ldots, x_{d}\right) .
$$


Let $e$ be a subset of natural numbers in $[1, d]$. We denote

$$
\Delta_{\mathbf{t}}^{l}(e)=\prod_{j \in e} \Delta_{t_{j}, j}^{l}, \quad \Delta_{\mathbf{t}}^{l}(\varnothing)=I
$$

We define the class $\mathbf{H}_{p, l}^{r} B, l>r$, as the set of $f \in L_{p}$ such that for any $e$

$$
\left\|\Delta_{\mathbf{t}}^{l}(e) f(\mathbf{x})\right\|_{p} \leq B \prod_{j \in e}\left|t_{j}\right|^{r} .
$$

In the case $B=1$ we omit it. It is known (see, for instance, [157], p.137) that the classes $\mathbf{H}_{p, l}^{r}$ with different $l$ are equivalent. So, for convenience we fix one $l=[r]+1$ and omit $l$ from the notation.

There are constructive methods for the sampling recovery based on sparse grids (Smolyak point sets $S G(n)$ ) (see [39], Chapter 5 and [157], Section 6.9). For instance, these methods give the following upper bounds for the sampling recovery for all $r>1 / p, 2 \leq p \leq \infty$ (see [157], p.307, Theorem 6.9.2).

$$
\varrho_{m}\left(\mathbf{H}_{p}^{r}, L_{2}\right) \leq C(r, p, d) m^{-r}(\log m)^{(d-1)(1+r)} .
$$

It is also known that in many cases sparse grid methods are optimal sampling recovery methods for classes of functions with mixed smoothness ([157], Section 6.9.3). However, results of [162] show that it is not the case for the small smoothness situation. It is proved in [162] with the aid of (2.18): Let $2<p \leq \infty$ and $1 / p<r<1 / 2$ then

$$
\varrho_{m}\left(\mathbf{H}_{p}^{r}, L_{2}\right) \leq C(r, p, d) m^{-r}(\log m)^{d-1+r}
$$

and in the case $r=1 / 2$

$$
\varrho_{m}\left(\mathbf{H}_{p}^{1 / 2}, L_{2}\right) \leq C(r, p, d) m^{-1 / 2}(\log m)^{d-1 / 2}(\log \log m)^{3 / 2} .
$$

It follows from known results (see, for instance, [40], [157], Section 6.4, and a discussion in [162]) that the upper bound (2.22) cannot be improved if we use the $(n, n-1)$-nets, in particular the sparse grids (see [157], Section 6.4 for their definitions), as sample sets for recovery. Comparing above mentioned lower bounds for (2.22) and inequalities (2.23) and (2.24), we conclude that, in the range of parameters $1 / p<r \leq 1 / 2,2<p<\infty, d>2$, there exists a weighted least squares algorithm, which provides better (albeit, nonconstructive) upper bounds for sampling recovery than algorithms based on sparse grids or even based on a wider class of point sets - the $(n, n-1)$-nets. 


\subsection{Some comments on techniques}

It turns out that the technique used in sampling discretization is rather rich and connected with deep techniques from different areas of mathematics functional analysis, harmonic analysis, probability, and others. In brief, here are a few of these connections.

T.1. The result in D.1 is based on a simple well known identity:

$$
\sum_{l=0}^{2 n} e^{i k 2 \pi l /(2 n+1)}= \begin{cases}2 n+1, & k=0, \\ 0, & 0<|k| \leq 2 n .\end{cases}
$$

T.2. Result D.3 is based on the Riesz-Thorin interpolation theorem (see, for instance, [157], Sections 1.3.3 and 3.3.4).

T.3. Sampling discretization for the hyperbolic cross polynomials $\mathcal{T}\left(Q_{n}\right)$ turns out to be a very difficult and interesting problem. It may serve as a testing field for different methods. These results were obtained with the aid of probabilistic techniques, in particular: a variant of the Bernstein concentration measure inequality from [19] (see Lemma 2.1 below), the chaining technique from [85] (see also [153], Ch.4), and bounds of the entropy numbers. The reader can find further results on the chaining technique in [148].

We formulate a lemma, which is an analog of the well known concentration measure inequalities (see, for instance [153], Ch.4). Lemma 2.1 is from [19].

Lemma 2.1. Let $\left\{g_{j}\right\}_{j=1}^{m}$ be independent random variables with $\mathbb{E} g_{j}=0$, $j=1, \ldots, m$, which satisfy

$$
\left\|g_{j}\right\|_{1} \leq 2, \quad\left\|g_{j}\right\|_{\infty} \leq M, \quad j=1, \ldots, m
$$

Then for any $\eta \in(0,1)$ we have the following bound on the probability

$$
\mathbb{P}\left\{\left|\sum_{j=1}^{m} g_{j}\right| \geq m \eta\right\}<2 \exp \left(-\frac{m \eta^{2}}{8 M}\right) .
$$

This lemma, in combination with different chaining techniques and bounds on the entropy numbers of the unit $L_{q}$-ball in the uniform norm, was used in D.4, D.6, D.10, and D.13.

T.4. For all $q \in[1, \infty)$ distinct from 2 , we obtain the sampling discretization of the $L_{q}$ norm results close to the ones optimal in the sense of order. The necessary condition for successful discretization in an $N$-dimensional 
subspace is $m \geq N$. The results in $\mathbf{D . 1 6}$ and $\mathbf{D . 1 7}$ provide good discretization for $m$ of order $N(\log N)^{a}$ with $a>0$ depending on the subspace. It seems like the probability technique used does not allow us to get rid of the logarithmic factor in the bound for $m$. It turns out that in the case $q=2$ we can get rid of the logarithmic factor and obtain optimal (in the sense of order) results. This technique is based on results by A. Marcus, D.A. Spielman, and N. Srivastava from [99] (see Corollary 1.5 with $r=2$ there) obtained for solving the Kadison-Singer problem (see Theorem 1.1 from Section 1). We now discuss this technique.

The following simple technical result (see [33], Proposition 2.1) illustrates reduction of a continuous problem to the discrete one.

Theorem 2.1. Let $Y_{N}:=\operatorname{span}\left\{u_{1}(x), \ldots, u_{N}(x)\right\}$ with $\left\{u_{i}(x)\right\}_{i=1}^{N}$ being a real (or complex) orthonormal on $\Omega$ with respect to a probability measure $\mu$ basis for $Y_{N}$. Assume that $\left\|u_{i}\right\|_{4}:=\left\|u_{i}\right\|_{L_{4}(\Omega, \mu)}<\infty$ for all $i=1, \ldots, N$. Then for any $\delta>0$ there exists a set $\Omega_{M}=\left\{x^{j}\right\}_{j=1}^{M} \subset \Omega$ such that for any $f \in Y_{N}$

$$
\left|\|f\|_{L_{2}(\Omega)}^{2}-\|f\|_{L_{2}\left(\Omega_{M}\right)}^{2}\right| \leq \delta\|f\|_{L_{2}(\Omega)}^{2}
$$

where

$$
\|f\|_{L_{2}\left(\Omega_{M}\right)}^{2}:=\frac{1}{M} \sum_{j=1}^{M}\left|f\left(x^{j}\right)\right|^{2} .
$$

We now discuss some lemmas, that are used for solving the Marcinkiewicztype discretization problem in $L_{2}$ for discrete sets. The following Lemma 2.2 (see [56], Corollary E.2) by N.J. Harvey and N. Olver was derived from Theorem 1.1.

Lemma 2.2. Let $\mathbf{v}_{1}, \ldots, \mathbf{v}_{M} \in \mathbb{R}^{N}$ be such that for all $\mathbf{w} \in \mathbb{C}^{N}$

$$
\alpha\|\mathbf{w}\|_{2}^{2} \leq \sum_{j=1}^{M}\left|\left\langle\mathbf{w}, \mathbf{v}_{j}\right\rangle\right|^{2} \leq \beta\|\mathbf{w}\|_{2}^{2}
$$

and $\left\|v_{j}\right\|^{2}=\delta:=N / M$ for all $j$. Suppose that $\alpha \in[1 / 2,1]$ and $\beta \in[1,2]$. Then there exists $J \subset\{1,2, \ldots, M\}$ satisfying

$$
(\alpha-C \sqrt{\delta})\|\mathbf{w}\|_{2}^{2} \leq 2 \sum_{j \in J}\left|\left\langle\mathbf{w}, \mathbf{v}_{j}\right\rangle\right|^{2} \leq(\beta+C \sqrt{\delta})\|\mathbf{w}\|_{2}^{2},
$$

where $C$ is an absolute constant $C \geq 1$. 
The following Lemma 2.3 (see Lemma 2 in [109] and also see [116], Lemma 10.22 , p.105) was proved with the aid of Lemma 2.2 by using the iteration technique which is discussed in Subsection 4.2.

Lemma 2.3. Let a system of vectors $\mathbf{v}_{1}, \ldots, \mathbf{v}_{M}$ from $\mathbb{C}^{N}$ satisfy (1.9) for all $\mathbf{w} \in \mathbb{C}^{N}$ and

$$
\left\|\mathbf{v}_{j}\right\|_{2}^{2}=N / M, \quad j=1, \ldots, M .
$$

Then there is a subset $J \subset\{1,2, \ldots, M\}$ such that for all $\mathbf{w} \in \mathbb{C}^{N}$

$$
c_{0}\|\mathbf{w}\|_{2}^{2} \leq \frac{M}{N} \sum_{j \in J}\left|\left\langle\mathbf{w}, \mathbf{v}_{j}\right\rangle\right|^{2} \leq C_{0}\|\mathbf{w}\|_{2}^{2},
$$

where $c_{0}$ and $C_{0}$ are some absolute positive constants.

A simple Remark 2.1 is from [154].

Remark 2.1. For the cardinality of the subset $J$ from Lemma 2.3 we have

$$
c_{0} N \leq|J| \leq C_{0} N
$$

We note that the iteration technique was already used in the paper [56] in order to obtain the following result (see Claim E.3 in [56]), which is similar to Lemma 2.3). Here we give a slight reformulation of it, which follows from the proof of Claim E.3 in [56].

Lemma 2.4. Let a system of vectors $\mathbf{v}_{1}, \ldots, \mathbf{v}_{M}$ from $\mathbb{R}^{N}$ satisfy (1.9) for all $\mathbf{w} \in \mathbb{R}^{N}$ and

$$
\left\|\mathbf{v}_{j}\right\|_{2}^{2}=N / M, \quad j=1, \ldots, M .
$$

For any $\varepsilon \leq 1 / 9$ there is a subset $J \subset\{1,2, \ldots, M\}$ with $|J| \leq C_{1} N / \varepsilon^{2}$, where $C_{1}$ is a positive absolute constant, such that for all $\mathbf{w} \in \mathbb{R}^{N}$

$$
(1-\varepsilon)\|\mathbf{w}\|_{2}^{2} \leq \frac{M}{|J|} \sum_{j \in J}\left|\left\langle\mathbf{w}, \mathbf{v}_{j}\right\rangle\right|^{2} \leq(1+\varepsilon)\|\mathbf{w}\|_{2}^{2} .
$$

The following Lemma 2.5, which is a generalization of Lemma 2.3, and Corollary 2.1 were proved in [96].

Lemma 2.5. Let a system of vectors $\mathbf{v}_{1}, \ldots, \mathbf{v}_{M}$ from $\mathbb{C}^{N}$ satisfy (1.9) for all $\mathbf{w} \in \mathbb{C}^{N}$ and

$$
\left\|\mathbf{v}_{j}\right\|_{2}^{2} \leq \theta N / M, \quad \theta \leq M / N, \quad j=1, \ldots, M
$$


Then there is a subset $J \subset\{1,2, \ldots, M\}$ such that for all $\mathbf{w} \in \mathbb{C}^{N}$

$$
c_{0} \theta\|\mathbf{w}\|_{2}^{2} \leq \frac{M}{N} \sum_{j \in J}\left|\left\langle\mathbf{w}, \mathbf{v}_{j}\right\rangle\right|^{2} \leq C_{0} \theta\|\mathbf{w}\|_{2}^{2}, \quad|J| \leq C_{1} \theta N
$$

where $c_{0}, C_{0}$, and $C_{1}$ are some absolute positive constants.

We note that the proof of Lemma 2.5 in [96] gives a slightly stronger result than Lemma 2.5 - the tight frame condition (1.9) can be replaced by a frame condition

$$
A\|\mathbf{w}\|_{2}^{2} \leq \sum_{j=1}^{M}\left|\left\langle\mathbf{w}, \mathbf{v}_{j}\right\rangle\right|^{2} \leq B\|\mathbf{w}\|_{2}^{2}
$$

This stronger version of Lemma 2.5 was formulated in the followup paper [110], where it was used for sampling recovery. Also, [110] gives a proof with specified constants $c_{0}, C_{0}$, and $C_{1}$.

Corollary 2.1. Let a system of vectors $\mathbf{v}_{1}, \ldots, \mathbf{v}_{M}$ from $\mathbb{C}^{N}$ satisfy (1.9) for all $\mathbf{w} \in \mathbb{C}^{N}$. Then there exists a set of weights $\lambda_{j} \geq 0, j=1, \ldots, M$, such that $\left|\left\{j: \lambda_{j} \neq 0\right\}\right| \leq 2 C_{1} N$ and for all $\mathbf{w} \in \mathbb{C}^{N}$ we have

$$
c_{0}\|\mathbf{w}\|_{2}^{2} \leq \sum_{j=1}^{M} \lambda_{j}\left|\left\langle\mathbf{w}, \mathbf{v}_{j}\right\rangle\right|^{2} \leq C_{0}\|\mathbf{w}\|_{2}^{2} .
$$

where $c_{0}, C_{0}$, and $C_{1}$ are absolute positive constants from Lemma 2.5.

Lemma 2.3 was used in D.5 and D.9. Lemma 2.5 was used in D.15 and Corollary 2.1 was used in D.18. We point out that results based on Lemma 2.5 and Corollary 2.1 found their applications in the optimal recovery problems (see [110] and [161]).

T.5. In [86] Talagrand's generic chaining and its development due to van Handel (see [55]) have been applied. A key step in the proofs by chaining is to bound certain entropy numbers. In [86] this was accomplished by the following theorem (see [148], p.552, Lemma 16.5.4). For a Banach space $X$ we define the modulus of smoothness

$$
\rho(u):=\rho(X, u):=\sup _{\|x\|=\|y\|=1}\left(\frac{1}{2}(\|x+u y\|+\|x-u y\|)-1\right) .
$$


Let $\mathcal{D}_{n}=\left\{g_{j}\right\}_{j=1}^{n}$ be a system of elements of cardinality $\left|\mathcal{D}_{n}\right|=n$ in a Banach space $X$. We equip the linear space $W_{n}:=\left[\mathcal{D}_{n}\right]:=\operatorname{span}\left\{\mathcal{D}_{n}\right\}$ with the norm

$$
\|f\|_{A}:=\|f\|_{A_{1}\left(\mathcal{D}_{n}\right)}:=\inf \left\{\sum_{j=1}^{n}\left|c_{j}\right|: f=\sum_{j=1}^{n} c_{j} g_{j}\right\} .
$$

Denote by $W_{n, A}$ the $W_{n}$ equipped with the norm $\|\cdot\|_{A}$. We are interested in the dual norm to the norm $\|\cdot\|_{A}$, which we denote $\|\cdot\|_{U}$ :

$$
\|F\|_{U}:=\|F\|_{U\left(\mathcal{D}_{n}\right)}:=\sup _{f \in W_{n} ;\|f\|_{A} \leq 1}|F(f)| .
$$

Denote $W_{n, U}^{*}$ the $W_{n}^{*}$ equipped with the norm $\|\cdot\|_{U}$. Note that $\|\cdot\|_{U}$ is a semi-norm on the dual to $X$, space $X^{*}$.

Theorem 2.2. Let $X$ be q-smooth: $\rho(X, u) \leq \gamma u^{q}, 1<q \leq 2$ and let $\mathcal{D}_{n}$ be a normalized system in $X$ of cardinality $\left|\mathcal{D}_{n}\right|=n$. Then for the unit ball $B\left(X^{*}\right)$ of $X^{*}$ we have

$$
\varepsilon_{k}\left(B\left(X^{*}\right),\|\cdot\|_{U\left(\mathcal{D}_{n}\right)}\right) \leq C(X)\left(\frac{\log n}{k}\right)^{1-1 / q}, \quad k=1, \ldots, n .
$$

We note that Talagrand's inequality (2.25) was slightly improved in [160], where a different technique, based on a combination of the deep duality result for the entropy numbers proved in [20] and results from greedy approximation, was used: The factor $\log n$ was replaced by $\log (2 n / k)$. The following result from [86], was proved with the aid of Theorem 2.2.

Lemma 2.6. Let $q \in(2, \infty)$. Assume that for any $f \in X_{N}$ we have

$$
\|f\|_{\infty} \leq M\|f\|_{q}
$$

with some constant $M$. Then for $k \in[1, N]$ we have for any finite subset $Y_{s} \subset \Omega$ of $s$ points

$$
\varepsilon_{k}\left(X_{N}^{q}, L_{\infty}\left(Y_{s}\right)\right) \leq C(q)(\log s)^{1 / q}\left(M N^{-1 / q}\right)(N / k)^{1 / q} .
$$

This technique was used in D.14 and D.17.

We now comment on the relation between the Nikol'skii inequality condition (2.26) and the entropy condition (2.6). On one hand it is easy to see 
(see [35]) that the entropy condition (2.6) for $k=1$ implies the following Nikol'skii inequality

$$
\|f\|_{\infty} \leq 4 B N^{1 / q}\|f\|_{q} \text { for all } f \in X_{N} .
$$

On the other hand, we note that the Nikol'skii-type inequality condition (2.26) with $M=B N^{1 / q}$ implies the entropy condition (2.6) with $k=1$. Thus, the Nikol'skii-type inequality condition is equivalent to the the entropy condition (2.6) for $k=1$. Moreover, Lemma 2.6 shows that the Nikol'skii inequality (2.26) implies the entropy condition in the $L_{\infty}\left(Y_{s}\right)$ norm for the whole range $k \in[1, N]$. Further, the following Lemma 2.7 from [159] shows that the Nikol'skii inequality (2.26), combined with an extra mild condition, implies the entropy condition in the $L_{\infty}$ norm.

Lemma 2.7. Let $q \in(2, \infty)$. Assume that for any $f \in X_{N}$ we have

$$
\|f\|_{\infty} \leq B N^{1 / q}\|f\|_{q}
$$

with some constant $B$. Also, assume that $X_{N} \in \mathcal{M}\left(s, \infty, C_{1}\right)$ with $s \leq a N^{c}$. Then for $k \in[1, N]$ we have

$$
\varepsilon_{k}\left(X_{N}^{q}, L_{\infty}\right) \leq C\left(q, a, c, C_{1}\right)(\log N)^{1 / q} B(N / k)^{1 / q} .
$$

\subsection{Some further comments and open problems}

In Subsections 2.1-2.4 we provided some sufficient conditions for the Marcinkiewicz-type theorem to hold, but optimal conditions would be more useful. We now formulate the corresponding optimization problem the formulation of which is the same in both the Marcinkiewicz $\mathcal{M}\left(m, L_{q}(\Omega, \mu), C_{1}, C_{2}\right)$ theorem and in its weighted analog $\mathcal{M}^{w}\left(m, L_{q}(\Omega, \mu), C_{1}, C_{2}\right)$. For an $N$-dimensional subspace $X_{N} \subset L_{q}(\Omega, \mu), 1 \leq q<\infty$, define

$$
m^{w}\left(X_{N}, L_{q}(\Omega, \mu), C_{1}, C_{2}\right):=\inf \left\{m: X_{N} \in \mathcal{M}^{w}\left(m, q, C_{1}, C_{2}\right)\right\}
$$

and

$$
s d^{w}\left(N, q, C_{1}, C_{2}\right):=\sup _{X_{N}, \Omega, \mu} m^{w}\left(X_{N}, L_{q}(\Omega, \mu), C_{1}, C_{2}\right) .
$$

Here $s d$ stands for the sampling discretization. In the above definition of $s d^{w}\left(N, q, C_{1}, C_{2}\right)$ and in the similar definition of $s d\left(N, q, C_{1}, C_{2}\right)$ we take supremum over all $N$-dimensional subspaces of $L_{q}(\Omega, \mu)$. In the case when $\Omega$ 
and $\mu$ are fixed and $N$-dimensional subspaces of $L_{q}(\Omega, \mu)$ come from a special collection $\mathcal{F}$ we write

$$
s d^{w}\left(\mathcal{F}, N, L_{q}(\Omega, \mu), C_{1}, C_{2}\right):=\sup _{X_{N} \in \mathcal{F}} m^{w}\left(X_{N}, L_{q}(\Omega, \mu), C_{1}, C_{2}\right) .
$$

We are interested in behavior of characteriscics $s d^{w}\left(N, q, C_{1}, C_{2}\right)$ and their analogs when $N$ goes to infinity and the constants $C_{1}$ and $C_{2}$ are fixed (do not depend on $N$ ). Certainly, this behavior may depend on a specific pair of constants $C_{1}$ and $C_{2}$. In order to focus attention on dependence on $N$ we talk about a generic characteristic $s d^{w}(N, q)$, which we do not define formally.

Definition 2.2. We say that the sequence of sampling weighted discretization numbers $s d^{w}(N, q)$ grows as $\varphi(N)$ with $N \rightarrow \infty$ if there exist three positive numbers $C_{i}^{*}(q), i=1,2,3$, which may depend on $q$ such that

$$
s d^{w}\left(N, q, C_{1}^{*}(q), C_{2}^{*}(q)\right) \leq C_{3}^{*}(q) \varphi(N), \quad N \in \mathbb{N},
$$

and for any $0<C_{1} \leq C_{2}<\infty$ there is a constant $C\left(C_{1}, C_{2}\right)>0$ such that we have

$$
s d^{w}\left(N, q, C_{1}, C_{2}\right) \geq C\left(C_{1}, C_{2}\right) \varphi(N), \quad N \in \mathbb{N} .
$$

We use similar definitions for the sequences

$$
\{s d(N, q)\}, \quad\left\{s d\left(\mathcal{F}, N, L_{q}(\Omega, \mu)\right)\right\}, \quad \text { and } \quad\left\{s d^{w}\left(\mathcal{F}, N, L_{q}(\Omega, \mu)\right)\right\} .
$$

It is clear that for all characteristics mentioned above we have $\varphi(N) \geq N$.

Main Problem. The main problem of sampling discretization is to find the growth rate of the sequences $\{s d(N, q)\},\left\{s d^{w}(N, q)\right\}$ for all $1 \leq q<\infty$, the growth rate of the sequence $\{s d(N, \infty)\}$, and the growth rate of the sequences $\left\{s d\left(\mathcal{F}, N, L_{q}(\Omega, \mu)\right)\right\}$ and $\left\{s d^{w}\left(\mathcal{F}, N, L_{q}(\Omega, \mu)\right)\right\}$ for $1 \leq q<\infty$, $\left\{s d\left(\mathcal{F}, N, L_{\infty}(\Omega, \mu)\right)\right\}$ for special collections $\mathcal{F}$.

We point out that the Main Problem is solved in only a few cases. Results of D.18 claim that the sequence $\left\{s d^{w}(N, 2)\right\}$ grows as $N$. Results of D.15 claim that for any $\Omega$ and $\mu$ the sequence $\left\{\operatorname{sd}\left(\mathcal{N}\left(2, \infty, t N^{1 / 2}\right), N, L_{2}(\Omega, \mu)\right)\right\}$ grows as $N$. Here

$$
\mathcal{N}(p, \infty, M):=\left\{X_{N}:\|f\|_{\infty} \leq M\|f\|_{p}, \quad \forall f \in X_{N} \subset L_{p}(\Omega, \mu)\right\} .
$$

Another important collection of subspaces is the collection $\mathcal{E}(N, q, B)$ of $N$-dimensional subspaces satisfying the entropy condition (2.6). 
Open problem 1. Find the growth rate of the sequence $\{s d(N, q)\}, q \in[1, \infty]$.

Open problem 2. Find the growth rate of the sequence $\left\{s d^{w}(N, q)\right\}, q \in[1, \infty) \backslash\{2\}$.

Open problem 3. Find the growth rate of the sequence $\left\{s d\left(\mathcal{N}\left(2, \infty, t N^{1 / 2}\right), N, L_{q}(\Omega, \mu)\right)\right\}, q \in[1, \infty) \backslash\{2\}$.

Open problem 4 . Find the growth rate of the sequence $\left\{s d\left(\mathcal{N}\left(q, \infty, t N^{1 / q}\right), N, L_{q}(\Omega, \mu)\right)\right\}, q \in[1, \infty) \backslash\{2\}$.

Open problem 5. Find the growth rate of the sequence $\left\{\operatorname{sd}\left(\mathcal{E}(N, q, B), N, L_{q}(\Omega, \mu)\right)\right\}, q \in[1, \infty) \backslash\{2\}$.

The problem of the growth rate of the $s d$-sequences is open even in the case of trigonometric polynomials. Denote for $\Omega=[0,2 \pi]^{d}$ and $\mu$ the normalized Lebesgue measure on $\Omega$

$$
\mathcal{T r}(N, d):=\left\{\mathcal{T}(Q): Q \subset \mathbb{Z}^{d},|Q|=N\right\}
$$

Open problem 6. Find the growth rate of the sequence $\left\{s d\left(\mathcal{T} r(N, d), N, L_{q}(\Omega, \mu)\right)\right\}, q \in[1, \infty) \backslash\{2\}$.

Results D.7, D.12, and D.21 show that the case $q=\infty$ is very different from the case $1 \leq q<\infty$.

Open problem 7 . Find the growth rate of the sequence $\left\{s d\left(\mathcal{T} r(N, d), N, L_{\infty}(\Omega, \mu)\right)\right\}$.

Note, that results $\mathbf{D . 1 2}$, and $\mathbf{D} . \mathbf{2 1}$ give

$$
e^{c_{1} N} \leq \operatorname{sd}\left(\mathcal{T} r(N, d), N, L_{\infty}(\Omega, \mu), 1 / 2\right) \leq e^{c_{2} N}
$$

where $c_{2} \geq c_{1}>0$ are absolute constants.

\section{Spectral properties and operator norms of submatrices}

Theorem 1.1 mentioned in Introduction is actually a theorem on partitioning a matrix into two submatrices with extremely small $(2,2)$-norms (see (1.6) for the definition). It can be reformulated as follows:

Theorem 3.1. Let $A$ be an $M \times N$ matrix with columns forming an orthonormal system in $\ell_{2}^{M}$ and rows $\mathbf{v}_{j}, j=1, \ldots, M$, such that $\left\|\mathbf{v}_{j}\right\|_{2} \leq \varepsilon$, for 
some $\varepsilon>0$. Then there exists a partition of the set $\langle M\rangle:=\{1,2, \ldots, M\}$ into two parts

$$
\langle M\rangle=\Omega_{1} \sqcup \Omega_{2}
$$

such that

$$
\left\|A\left(\Omega_{k}\right)\right\|^{2} \leq \frac{(1+\sqrt{2} \varepsilon)^{2}}{2}, \quad k=1,2,
$$

where for $\omega \subset\langle M\rangle$ we define $A(\omega)$ as a submatrix of $A$ obtained from $A$ by extracting the rows numbered in $\omega$.

For a matrix satisfying conditions of Theorem 3.1 inequality (3.2) is equivalent to the following pointwise estimate: For $k=1,2$

$$
\left\|A\left(\Omega_{k}\right) \mathbf{x}\right\|_{2}^{2} \leq \frac{(1+\sqrt{2} \varepsilon)^{2}}{2}\|A \mathbf{x}\|_{2}^{2}, \quad \forall \mathbf{x} \in \mathbb{R}^{N} .
$$

As we saw in Subsection 2.6, the best in the sense of order Marcinkiewicz-type discretization theorem for finite-dimensional subspaces in $L_{2}$ can be derived from Theorem 3.1. There is a chance that similar results for other norms of matrices can improve known Marcinkiewicz-type discretization theorems.

In this section we present known results on partitioning a matrix into two submatrices with extremely small $(p, q)$-norms (as in (3.2)) and with good pointwise estimate of the $\ell_{q}$ norm of the image of any vector (as in (3.3)) for different $1 \leq p, q \leq \infty$. More precisely, extremely small $(p, q)$-norm here is understood as the property of the norm raised to the power $q$ to be extremely close to the half. Let us comment on these two types of problems.

Let $A$ be an $M \times N$ matrix. We assume that $M \geq N$ and consider $A$ as an operator acting between $\mathbb{R}^{N}$ and $\mathbb{R}^{M}$. We start with the problem of partitioning of a matrix $A$ into two submatrices with good pointwise estimates for $\ell_{q}$ norm, $1 \leq q \leq \infty$, of the image of any vector. We say that partition (3.1) has a good pointwise estimate if the following inequality holds for $k=1,2$ :

$$
\left\|A\left(\Omega_{k}\right) \mathbf{x}\right\|_{q}^{q} \leq\left(\frac{1}{2}+\varphi(A)\right)\|A \mathbf{x}\|_{q}^{q}, \quad \forall \mathbf{x} \in \mathbb{R}^{N}
$$

with some parameter $0<\varphi(A)<1 / 2$ (depending only on $A$ ). Quality of the partition is measured by how small $\varphi(A)$ is. For a partition of the form (3.1) we obviously have

$$
\left\|A\left(\Omega_{1}\right) \mathbf{x}\right\|_{q}^{q}+\left\|A\left(\Omega_{2}\right) \mathbf{x}\right\|_{q}^{q}=\|A \mathbf{x}\|_{q}^{q}, \quad \forall \mathbf{x} \in \mathbb{R}^{N} .
$$


So, we cannot have the factor in the right-hand side of (3.4) less than $1 / 2$. For this reason, if inequality (3.4) or its analog for the norm is satisfied, then we say that the submatrix $A\left(\Omega_{k}\right)$ has extremely small norm.

Thus if (3.4) holds, then for $k=1,2$ we get

$$
\left(\frac{1}{2}-\varphi(A)\right)\|A \mathbf{x}\|_{q}^{q} \leq\left\|A\left(\Omega_{k}\right) \mathbf{x}\right\|_{q}^{q} \leq\left(\frac{1}{2}+\varphi(A)\right)\|A \mathbf{x}\|_{q}^{q}, \quad \forall \mathbf{x} \in \mathbb{R}^{N} .
$$

Actually, under some conditions on $A$ we can guarantee that $\varphi(A)$ is small enough (see, e.g. Theorem 1.1 and M.11). In this case we can find a submatrix $B$ of $A$ with the number of rows not exceeding $M / 2$ such that the quantities $2^{1 / q}\|B \mathbf{x}\|_{q}$ and $\|A \mathbf{x}\|_{q}$ are almost the same for all $\mathbf{x} \in \mathbb{R}^{N}$. In some cases we can continue and find a partition with good pointwise estimate for the matrix $B$ and so on (see Subsection 4.2 for details). This process was applied in a number of papers (see, e.g., [97], [109], [96], [110]). The idea of such an iteration process was proposed by Lunin in the remarkable paper [97], where he obtained the first sharp estimates (see M.1 for details).

Let $1 \leq p \leq \infty$. By taking the supremum over all $\mathbf{x} \in \mathbb{R}^{N}$ with $\|\mathbf{x}\|_{p} \leq 1$ in (3.4), we obtain the necessary condition for (3.4) to hold, namely, the following inequality:

$$
\left\|A\left(\Omega_{k}\right)\right\|_{(p, q)}^{q} \leq\left(\frac{1}{2}+\varphi(A)\right)\|A\|_{(p, q)}^{q}, \quad k=1,2 .
$$

This type of estimate is a different type of result compared to the pointwise estimate.

Another setting considered below concerns square matrices. Here problems are formulated not only on extracting rows of a matrix but simultaneous extraction of both rows and columns is allowed (see, e.g. results M.4 and M.6 below). For $\omega \subset\langle N\rangle$ let $R_{\omega}^{N}$ be an $N \times N$ matrix with entries $r_{i}^{j}$ such that $r_{i}^{i}=1$ for $i \in \omega$ and other entries equal to 0 . Note that for an $M \times N$ matrix $A$ and $\omega \subset\langle M\rangle$ the matrices $R_{\omega}^{M} A$ and $A(\omega)$ have the same non-zero rows. Let $A$ be an $N \times N$ matrix. The goal is to find $\omega \subset\langle N\rangle$ such that $\left\|R_{\omega}^{N} A R_{\omega}^{N}\right\|$ is small enough. Due to a decoupling argument (see [21, Proposition 1.9]), this can be achieved if we have good estimates for the average of the quantity $\left\|R_{\omega}^{N} A R_{\omega^{\prime}}^{N}\right\|$ over all $\omega, \omega^{\prime} \subset\langle N\rangle$.

\subsection{Euclidean case}

We begin our discussion with the case that is most important for applications: 
The matrix $A$ is considered to be an operator acting between $\ell_{2}^{N}$ and $\ell_{2}^{M}$.

M.1. The first result on operator norms of submatrices is due to Kashin [66]: For any $\varepsilon>0$ there exists $C(\varepsilon)>0$ such that for any $M \times N$ matrix $A$ with $\|A\|=1$ and $M \geq C(\varepsilon) N$ there exists an $N \times N$ submatrix $A(\omega)$ with $\|A(\omega)\| \leq \varepsilon$. Later, in [97] Lunin established the following sharp estimate of $C(\varepsilon): C(\varepsilon) \leq B \varepsilon^{-2}$. Lunin's result is actually an estimate of the form (1.7) for arbitrary subspaces in $L_{2}(\Omega)$.

In [21] Bourgain and Tzafriri obtained results M.2 and M.3, which are widely known as the restricted invertibility theorems.

M.2. For some absolute positive constants $C_{1}$ and $C_{2}$ and an $N \times N$ matrix $A$ with $\left\|A\left(\mathbf{e}_{i}\right)\right\|_{2}=1, i=1, \ldots, N,\left(\left\{\mathbf{e}_{i}\right\}_{i=1}^{N}\right.$ is the standard basis of $\left.\mathbb{R}^{N}\right)$ there exists a set $\omega \subset\langle N\rangle$ of cardinality $|\omega| \geq C_{1} N /\|A\|^{2}$, such that

$$
\left\|A R_{\omega}^{N} \mathbf{x}\right\|_{2} \geq C_{2}\left\|R_{\omega}^{N} \mathbf{x}\right\|_{2}, \quad \forall \mathbf{x} \in \mathbb{R}^{N}
$$

M.3. For every $C>0$ and $0<\varepsilon<1$, there is a constant $d=d(C, \varepsilon)>0$ such that, whenever $N \geq 1 / d$ and $A$ is an $N \times N$ matrix with $\|A\| \leq C$ and $\left\langle A \mathbf{e}_{i}, \mathbf{e}_{i}\right\rangle=1, i=1, \ldots, N$, there exists $\omega \subset\langle N\rangle$ of cardinality $|\omega| \geq d N$ so that $R_{\omega}^{N} A R_{\omega}^{N}$ is invertible and

$$
\left\|\left(R_{\omega}^{N} A R_{\omega}^{N}\right)^{-1}\right\|<(1-\varepsilon)^{-1} .
$$

M.4. Here is a result from [79] on square matrices: Let $A$ be an $N \times N$ matrix. There exists a constant $C$ such that if $1 / N<\varepsilon<1,1 \leq r \leq N$, then

$$
\min _{\left|\omega_{1}\right|,\left|\omega_{2}\right| \geq \varepsilon N / 4}\left\|R_{\omega_{1}}^{N} A R_{\omega_{2}}^{N}\right\| \leq C\left(a_{r}(A) \varepsilon^{1 / 2}+\varepsilon+\left(\frac{\varepsilon r}{N}\right)^{1 / 2}\right)\|A\|,
$$

where $a_{r}(\omega)=\min \{\|A-B\|, B$ is an $N \times N$ matrix, rank $B \leq r\}$. The proof relies on averaging the quantity $\left\|R_{\omega_{1}}^{N} A R_{\omega_{2}}^{N}\right\|_{(\infty, 1)}$ and using Grothendieck factorization. By a decoupling argument the authors obtained a similar result with $\omega_{1}=\omega_{2}$ in (3.5) (see Corollary 6 in [79]) for $A$ of rank $A=r$ with zeros on the diagonal.

M.5. Here is the result from [129] due to Rudelson (see also D.15, MM.1): Let $t \geq 1$ and $A=\left[a_{i}^{j}\right], j=1, \ldots, M, i=1, \ldots, N, M>N$, be a matrix with orthonormal columns. Suppose that for all $j$

$$
\sqrt{\frac{M}{N}} \cdot\left(\sum_{i=1}^{N}\left(a_{i}^{j}\right)^{2}\right)^{1 / 2} \leq t
$$


Then for every $\varepsilon>0$ there exists a set $\omega \subset\langle M\rangle$ so that

$$
|\omega| \leq C \frac{t^{2}}{\varepsilon^{2}} N \log \frac{N t^{2}}{\varepsilon^{2}}
$$

and for all $\mathbf{x} \in \mathbb{R}^{N}$

$$
(1-\varepsilon)\|\mathbf{x}\| \leq \sqrt{\frac{M}{|\omega|}}\left\|R_{\omega}^{M} A \mathbf{x}\right\| \leq(1+\varepsilon)\|\mathbf{x}\| .
$$

An example from [79] shows that estimate (3.6) is the best one can obtain by the random selection method. The obstacle here is that in contrast to the upper estimate (see [21]) the lower estimate does not hold in average.

The Kadison-Singer problem has a number of equivalent formulations. It was solved in [99] by the new method of interlacing polynomials through proving Weaver's conjecture (see the stronger formulation of it in Theorems 1.1, 3.1, M.7).

M.6. The following quantitative version of Anderson's paving conjecture [2], which is equivalent to the Kadison-Singer problem, was also proved in [99]: For any $\varepsilon>0$, every zero-diagonal complex self-adjoint $N \times N$ matrix $T$ can be $(r, \varepsilon)$-paved with $r=(6 / \varepsilon)^{4}$. This means that there are coordinate projections $P_{1}, \ldots, P_{r}$ such that $\sum_{i=1}^{r} P_{i}=I_{N}$ (identical matrix) and $\left\|P_{i} T P_{i}\right\|_{(2,2)} \leq \varepsilon\|T\|_{(2,2)}$ for all $i=1, \ldots, r$.

M.7. We give here another formulation of Theorem 3.1 due to N. Srivastava (see [146]): For any vectors $\mathbf{v}_{1}, \ldots, \mathbf{v}_{M} \in \mathbb{R}^{N}$ satisfying:

$$
\left|\left\langle\mathbf{v}_{i_{0}}, \mathbf{x}\right\rangle\right|^{2} \leq \varepsilon^{2} \sum_{i=1}^{M}\left|\left\langle\mathbf{v}_{i}, \mathbf{x}\right\rangle\right|^{2}, \quad \forall \mathbf{x} \in \mathbb{R}^{N}, \quad \forall i_{0} \in\langle M\rangle,
$$

there is a partition of the form (3.1) such that for $k=1,2$

$$
\sum_{i \in \Omega_{k}}\left|\left\langle\mathbf{v}_{i}, \mathbf{x}\right\rangle\right|^{q} \leq\left(\frac{1}{2}+O(\varepsilon)\right) \sum_{i=1}^{M}\left|\left\langle\mathbf{v}_{i}, \mathbf{x}\right\rangle\right|^{q}, \quad q=2, \quad \forall \mathbf{x} \in \mathbb{R}^{N} .
$$

In other words, every quadratic form of this kind in which no term has too much influence can be divided into two quadratic forms which approximate it. 
M.8. We present the result from [119] which is applied to accelerate classical numerical methods for solving linear systems: There exists an absolute constant $C$ such that for any $N \times N$ hermitian matrix $A$ there exists a permutation $\sigma$ of the set $\langle N\rangle$ for which

$$
\left\|L_{\sigma}\right\| \leq C\|A\|,
$$

where $L_{\sigma}$ is a strictly lower triangular part of $P_{\sigma} A P_{\sigma}^{T}\left(P_{\sigma}\right.$ here denotes the associated $N \times N$ row permutation matrix). Anderson's paving conjecture was used to prove this.

\section{$3.2 p=2, q=1$}

Results for the case $p=2, q=1$ are interesting by themselves and they allow us to obtain important results for the Euclidean case by using the Grothendieck factorization theorem.

M.9. Lunin established the following sharp estimate in [97]: There is a constant $C$ such that for any $M \times N$ matrix there exists $\omega \subset\langle M\rangle$ with $|\omega|=N$ such that the following inequality holds:

$$
\|A(\omega)\|_{(2,1)} \leq C \frac{N}{M}\|A\|_{(2,1)} .
$$

Due to a factorization argument he deduced from this result the sharp estimate in M.1.

M.10. The following result from [79] on averaging slightly modifies the estimate from [23]: Let $\left\{\delta_{i}\right\}_{i=1}^{M}$ be a sequence of $\{0,1\}$-valued independent random variables of mean $0<\delta<1$ over some probability space $(\Omega, \mu)$ and for $\omega \in \Omega$ define $\Omega_{\omega}=\left\{1 \leq i \leq M: \delta_{i}(\omega)=1\right\}$. Then there exists a constant $D$ such that for $M \geq D$ and $1 \leq N \leq M$ we have

$$
\int_{\Omega}\left\|A\left(\Omega_{\omega}\right)\right\|_{(2,1)} d \mu \leq D\left(\delta M^{1 / 2}+(\delta N)^{1 / 2}\right)\|A\|_{(2,2)} .
$$

M.11. Let $I_{N}$ be the matrix of the identity operator in $\mathbb{R}^{N}$ and let $\varphi(\varepsilon):=5 \varepsilon^{1 / 2} \log ^{1 / 4}(3 / \varepsilon), 0<\varepsilon<1$. The following result from [71] is obtained by a modified approach proposed by Lunin in [97] (see also [70]): For some constant $\varepsilon_{0}>0$ and each $M \times N$ matrix $A$ such that $A^{*} A=I_{N}$ and the rows $\mathbf{v}_{j}, j \in\langle M\rangle$, of $A$ satisfy $\left\|\mathbf{v}_{j}\right\|_{2} \leq \varepsilon$ with $\varepsilon \leq \varepsilon_{0}$, there exists a partition 
of the form (3.1) such that both of the norms $\left\|A\left(\Omega_{1}\right)\right\|_{(2,1)}$ and $\left\|A\left(\Omega_{2}\right)\right\|_{(2,1)}$ do not exceed $(1 / 2+\varphi(\varepsilon))\|A\|_{(2,1)}$. This result is similar to Theorem 3.1 for $(2,2)$-norms but it does not allow us to make a good iteration process.

M.12. We mention here a slight generalization of the result from [73] (see also [94]) on the possibility of reducing $(2,1)$-norms of the matrix via partitioning into two submatrices: Let $1 \leq q<2,\|A\|_{(2, q)}=1,\left\|\mathbf{v}_{i}\right\|_{2} \leq \varepsilon$, $i \in\langle M\rangle$. Then there exists a partition of the form (3.1) with ||$\Omega_{1}|-| \Omega_{2}|| \leq 1$ such that

$$
\left\|A\left(\Omega_{k}\right)\right\|_{(2, q)} \leq \frac{1}{2^{\frac{1}{q}-\frac{1}{2}}}+2 \varepsilon, \quad k=1,2 .
$$

\subsection{General case}

In the case $(p, q) \notin\{(2,1),(2,2)\}$ no final (in the sense of order) results are known. The result M.9 is sharp, but there still remain important open questions in that problem, in particular, the question of obtaining two-sided inequalities is open.

M.13. We start with formulating the restricted invertibility theorem for the $(p, p)$-norm from [21]: For every $0<\varepsilon<1,1 \leq p \leq \infty$ and $B>0$, there exists a constant $c=c(\varepsilon, p, B)$ such that, whenever $N \geq 1 / c$ and $A$ is an $N \times N$ matrix with $\|A\|_{(p, p)} \leq B$ and ones on the diagonal, then one can find a subset $\sigma$ of $\{1,2, \ldots, N\}$ of cardinality $|\sigma| \geq c N$ so that $R_{\sigma}^{N} A R_{\sigma}^{N}$ is invertible and

$$
\left\|\left(R_{\sigma}^{N} A R_{\sigma}^{N}\right)^{-1}\right\|_{(p, p)} \leq(1-\varepsilon)^{-1} .
$$

For the rest of this section we consider the problem of partitioning a matrix into two submatrices with small norms and with good pointwise estimate. We start with the case of $(1, q)$-norm, $1 \leq q<\infty$. From convexity of the function $\|A(\mathbf{x})\|_{q}$ it is easy to see that $\|A\|_{(1, q)}$ is the maximal $\ell_{q}$-norm of the columns of $A$. Certainly, this quantity is much easier to deal with than in the case $1<p<\infty$, so here we need to impose a significantly weaker condition on the matrix $A$ to have a partition of a matrix with almost equal norms raised to the power $q$. Let

$$
\left|a_{i}^{j}\right| \leq \varepsilon\left\|\mathbf{w}_{i}\right\|_{q}, \quad i \in\langle N\rangle, \quad j \in\langle M\rangle,
$$

where $\mathbf{w}_{i}, i=1, \ldots, N$, are the columns of $A$.

M.14. We formulate positive results (for items a) and c) see [95], for item b) see [45] and [95]): Assume that, for an $M \times N$ matrix $A$, inequality 
(3.9) holds for some $0<\varepsilon<1$. Then there exists a partition of the form (3.1) such that, for $k=1,2$ the following inequalities hold:
a) $\left\|A\left(\Omega_{k}\right)\right\|_{(1, q)} \leq\left(\frac{1}{2}+\frac{3}{2} \varepsilon^{q / 3} \ln ^{1 / 3}(4 N)\right)^{1 / q}\|A\|_{(1, q)}$,
b) $\left\|A\left(\Omega_{k}\right)\right\|_{(1, q)} \leq\left(\frac{1}{2}+\frac{1}{2} \varepsilon^{q} \sqrt{M}\left(1+\log \left(\frac{N}{M}+1\right)\right)^{1 / 2}\right)^{1 / q}\|A\|_{(1, q)}$,
c) $\left\|A\left(\Omega_{k}\right)\right\|_{(1, q)} \leq\left(\frac{1+N \varepsilon^{q}}{2}\right)^{1 / q}\|A\|_{(1, q)}$.

Note that in the proof of c), the generalized ham sandwich theorem (see, e.g. [46]) was applied.

M.15. The negative result is also from [95]: For $N=2^{2 k-1}$, there exists a $2 k \times N$ matrix $A$ for which condition (3.9) with $\varepsilon^{q} \log _{2} 2 N>2$ is satisfied, and the following relation holds for any partition of the form (3.1):

$$
\max \left\{\left\|A\left(\Omega_{1}\right)\right\|_{(1, q)},\left\|A\left(\Omega_{2}\right)\right\|_{(1, q)}\right\}=\|A\|_{(1, q)} .
$$

Let $X$ be a normed space. We can define $\|A\|_{(X, q)}$ in the same way as in (1.6).

M.16. For $q=\infty$ and any matrix $A$ there is no partition into two submatrices with smaller $(X, q)$-norms, since the matrix $A$ has a row $\mathbf{v}_{\text {sup }}$ such that $\|A\|_{(X, \infty)}=\sup _{\|\mathbf{x}\|_{X} \leq 1}\left\langle\mathbf{x}, \mathbf{v}_{\text {sup }}\right\rangle$, and the norm of the submatrix containing the row $\mathbf{v}_{\text {sup }}$ is equal to the norm of the matrix $A$.

The following condition is a direct analog of condition (3.7) on a matrix in the case of arbitrary $q, 1 \leq q<\infty$ :

$$
\forall \mathbf{x} \in \mathbb{R}^{N} \quad \forall i_{0} \in\langle M\rangle \quad\left|\left\langle\mathbf{v}_{i_{0}}, \mathbf{x}\right\rangle\right| \leq \varepsilon\left(\sum_{i=1}^{M}\left|\left\langle\mathbf{v}_{i}, \mathbf{x}\right\rangle\right|^{q}\right)^{1 / q} .
$$

Note that for $1 \leq q_{1} \leq q_{2}$ condition (3.10) with $q=q_{2}$ is more restrictive than with $q=q_{1}$. It may happen that another condition on $A$ will be natural in the sense that it will lead to sharp estimates. We note that condition (3.10) is the $(q, \infty)$ Nikol'skii-type inequality. Srivastava asked in [146] whether there is an analog of the result $\mathbf{M . 7}$ under condition (3.10) for $q=1$.

M.17. A partially positive answer to Srivastava's question was obtained in [73] and then it was generalized to the case $1 \leq q<\infty$ in [95]. Very recently (see [87]) it was strengthened as follows: Assume that $A$ satisfies condition (3.10) for some $\varepsilon$ and $1 \leq q<\infty$ with $0<\varepsilon \leq N^{-1 / q}$. Then there exists a partition of the form (3.1) such that for any $\mathbf{x} \in \mathbb{R}^{N}$ and $k=1,2$ :

$$
\left\|A\left(\Omega_{k}\right) \mathbf{x}\right\|_{q}^{q} \leq \gamma\|A \mathbf{x}\|_{q}^{q}, \quad \gamma=\frac{1}{2}+C(q) \varepsilon^{q / 2} N^{1 / 2} .
$$


In terms of the quantity $\varphi(A)$, introduced in the beginning of Section 3 , this means that $\varphi(A) \leq C(q) \varepsilon^{q / 2} N^{1 / 2}$. Necessity of the condition $0<\varepsilon \leq N^{-1 / q}$, which was imposed in both [73] and [87], is an open problem.

M.18. The negative part of the answer to Srivastava's question consists of the following theorem from [73]: Let $N=2^{s}, s \in \mathbb{N}$, and $N^{-1 / 2} \leq \varepsilon \leq 1$. There exists a $2 N \times N$ matrix $A=A(N, \varepsilon)$ such that estimate (3.10) holds for any $\mathbf{x} \in \mathbb{R}^{N}$ and $i_{0} \in\langle 2 N\rangle$ but, nevertheless, the inequality

$$
\max \left(\left\|A\left(\Omega_{1}\right)\right\|_{(2,1)},\left\|A\left(\Omega_{2}\right)\right\|_{(2,1)}\right) \geq \frac{1}{\sqrt{2}}\left(\frac{1}{1+\left(\varepsilon N^{1 / 2}\right)^{-1}}\right)\|A\|_{(2,1)}
$$

holds for any partition (3.1) (with $M=2 N$ ). This result shows that (3.10) for $q=1$ does not guarantee existence of a partition of $A$ of the form (3.1) with a straightforward counterpart of property (3.8) for $q=1$. For its existence, it is necessary to assume that the parameter $\varepsilon$ tends to zero as $N \rightarrow \infty$. This example was recently generalized by Limonova to the case of $1 \leq q<4 / 3$.

M.19. Let $X$ be an $N$-dimensional normed space. One can easily obtain the following corollary of M.17 : Assume that, for an $M \times N$ matrix, condition (3.10) is satisfied for some $\varepsilon$ and $q$ with $0<\varepsilon \leq(\operatorname{rank}(A))^{-1 / q}$ and $1 \leq q<\infty$. Then there exists a partition of the form (3.1) such that, for $k=1,2$

$$
\left\|A\left(\Omega_{k}\right)\right\|_{(X, q)}^{q} \leq \gamma\|A\|_{(X, q)}^{q},
$$

where $\gamma$ is defined in M.17.

\subsection{Algorithms}

All of the results in Section 3 were initially established as existence results. Construction of efficient algorithms for finding desirable submatrices is an important problem. In this regard we note that the crucial difference between [5] and [99] is that in [5] there is a deterministic polynomial-time algorithm. Many researchers apply ideas from [5] to obtain algorithms for other problems. For example, Spielman and Srivastava suggested in [143] a short proof of the restricted invertibility theorem that contains a deterministic algorithm. 


\section{Connections with other problems}

\subsection{Moments of marginals of high-dimensional distri- butions}

Recall that a random vector $\mathbf{u}$ in $\mathbb{R}^{N}$ is a measurable function from some probability space $(F, \mathcal{F}, \mathbb{P})$ with values in $\mathbb{R}^{N}$. The distribution of the random vector $\mathbf{u}$ is a Borel measure $\mu$ on $\mathbb{R}^{N}$ such that $\mathbb{P}(\mathbf{u} \in A)=\mu(A)$ for each Borel set $A$. This also means that for any reasonable function (e.g. for any bounded continuous function) $\varphi: \mathbb{R}^{N} \rightarrow \mathbb{R}$ one has

$$
\mathbb{E} \varphi(\mathbf{u})=\int_{\mathbb{R}^{N}} \varphi(y) \mu(d y),
$$

where $\mathbb{E}$ denotes the expectation of a random variable, i.e., the Lebesgue integral with respect to the measure $\mathbb{P}$.

Consider a random vector $\mathbf{u}$ in $\mathbb{R}^{N}$. The main problem considered in this section is to understand how well one can approximate one-dimensional marginals of the distribution $\mathbf{u}$ (i.e. one-dimensional images of this distribution) by sampling. Consider $m$ independent copies $\mathbf{u}^{1}, \ldots, \mathbf{u}^{m}$ of the vector $\mathbf{u}$ and for $q \in[1, \infty)$ consider the random variable

$$
V_{q}(K):=\left.\sup _{y \in K}\left|\frac{1}{m} \sum_{j=1}^{m}\right|\left\langle y, \mathbf{u}^{j}\right\rangle\right|^{q}-\mathbb{E}|\langle y, \mathbf{u}\rangle|^{q} \mid,
$$

where $K \subset \mathbb{R}^{N}$. When $K=B_{2}$, where $B_{2}:=\left\{y \in \mathbb{R}^{N}:|y| \leq 1\right\}$ is the Euclidean ball, i.e. $|y|:=\sqrt{\langle y, y\rangle}$, we write $V_{q}$ in place of $V_{q}\left(B_{2}\right)$. One now wants to estimate the least possible number $m=m(\varepsilon, q, N)$ of sampling copies of vector $\mathbf{u}$ for which $V_{q}(K) \leq \varepsilon$ with high probability. This problem has been extensively studied for the last 20 years (see [1], [17], [44], [52], [53], [130], [147], [163], [169], [170] and citations therein).

On one hand, if

$$
K:=\left\{y \in \mathbb{R}^{N}: \mathbb{E}|\langle y, \mathbf{u}\rangle|^{q} \leq 1\right\}
$$

then the described problem of the approximation of one-dimensional marginals of the distribution can be seen as the special case of the sampling discretization problem, when $\mu$ is the distribution of $\mathbf{u}$ and the subspace $X_{N}:=\left\{\langle y, \cdot\rangle, y \in \mathbb{R}^{N}\right\} \subset L_{q}(\mu)$. On the other hand, for any $N$-dimensional 
subspace $X_{N}$ of $L_{q}(\Omega, \mu)$ one can take an orthonormal basis $u_{1}, \ldots, u_{N}$ in $X_{N}$ and consider the random vector $\mathbf{u}:=\left(u_{1}(x), \ldots, u_{N}(x)\right)$. Thus, the general sampling discretization results can be deduced from the probabilistic question about approximation of one-dimensional marginals of the distribution.

MM.1. The most studied case is $q=2$, in which the problem is equivalent to estimating of the number of samples $\mathbf{u}^{1}, \ldots, \mathbf{u}^{m}$ sufficient to approximate the covariance matrix $\mathbb{E} \mathbf{u} \otimes \mathbf{u}$ of a random vector by a sample covariance matrix $\frac{1}{m} \sum_{j=1}^{m} \mathbf{u}^{j} \otimes \mathbf{u}^{j}$. This problem is closely related to random matrix theory and the methods involved in studying it essentially depend on the properties of the distribution. Probably the first result of this type is the one from [7] on bounds for the norm of a random matrix with i.i.d. Bernoulli random entries with values \pm 1 . Similar results have already been discussed in Section 3 where random submatrices of a given matrix have been studied. The study of a sample covariance matrix was also partially motivated by the paper [64] where a fast algorithm for calculating the volume of a convex body has been constructed (see also the book [25]). For this algorithm one needs to calculate the covariance matrix of a random vector uniformly distributed on a convex set. The authors of [64] have shown that it is enough to take $m=c \frac{N^{2}}{\varepsilon}$ points to make $V_{2} \leq \varepsilon$ with high probability. J. Bourgain obtained in [17] that one can take $m \geq C(\varepsilon) N[\log N]^{3}$ for the same bound. Later M. Rudelson [130] improved Bourgain's bound to $m \geq C \frac{N}{\varepsilon^{2}}\left[\log \frac{N}{\varepsilon^{2}}\right]^{2}$ and Rudelson's bound was improved to $m \geq C(\varepsilon) N \log N$ in [120]. Actually, in [130] the following general theorem has been proved.

Theorem 4.1. There is a constant $C>0$ such that for any random vector $\mathbf{u}$ in $\mathbb{R}^{N}$, for which $|y|^{2}=\mathbb{E}|\langle y, \mathbf{u}\rangle|^{2}$ for every $y \in \mathbb{R}^{N}$, one has

$$
\mathbb{E} V_{2} \leq C \frac{\sqrt{\log N}}{\sqrt{m}}\left(\mathbb{E} \max _{1 \leq j \leq m}\left|\mathbf{u}^{j}\right|^{2}\right)^{1 / 2} \cdot\left(\left.\mathbb{E} \sup _{|y| \leq 1}\left|\frac{1}{m} \sum_{j=1}^{m}\right|\left\langle y, \mathbf{u}^{j}\right\rangle\right|^{2} \mid\right)^{1 / 2}
$$

where $\mathbf{u}^{1}, \ldots, \mathbf{u}^{m}$ are independent copies of the random vector $\mathbf{u}$.

One can easily verify that this theorem implies the following result (see e.g. the end of the proof of Theorem 3 in [52] or the end of the proof of Lemma 3.1 in [86]). 
Corollary 4.1. There is a constant $C>0$ such that for any random vector $\mathbf{u}$ in $\mathbb{R}^{N}$, for which $|y|^{2}=\mathbb{E}|\langle y, \mathbf{u}\rangle|^{2}$ for every $y \in \mathbb{R}^{N}$, one has

$$
\mathbb{E} V_{2} \leq C\left(\frac{\log N}{m} \mathbb{E} \max _{1 \leq j \leq m}\left|\mathbf{u}^{j}\right|^{2}+\frac{\sqrt{\log N}}{\sqrt{m}}\left(\mathbb{E} \max _{1 \leq j \leq m}\left|\mathbf{u}^{j}\right|^{2}\right)^{1 / 2}\right)
$$

where $\mathbf{u}^{1}, \ldots, \mathbf{u}^{m}$ are independent copies of the random vector $\mathbf{u}$.

For the general problem of sampling discretization this corollary means that for any $N$-dimensional subspace $X_{N}$ of $L_{2}(\Omega, \mu)$, such that for every element $f \in X_{N}$ one has $\|f\|_{\infty} \leq t N^{\frac{1}{2}}\|f\|_{2}$ (i.e. Condition E holds), the $\mathcal{M}(m, 2, \varepsilon)$ theorem holds for every $m \geq c \frac{N t^{2}}{\varepsilon^{2}} \log N$ for big enough constant $c$. Indeed, consider the random vector $\mathbf{u}:=\left(u_{1}(x), \ldots, u_{N}(x)\right)$, where $u_{1}, \ldots, u_{N}$ is an orthonormal in $L_{2}(\Omega, \mu)$ basis of $X_{N}$. We need to estimate $\mathbb{E} \max _{1 \leq j \leq m}\left|\mathbf{u}^{j}\right|^{2}$. We have $|\mathbf{u}|^{2}=\left|u_{1}(x)\right|^{2}+\ldots+\left|u_{N}(x)\right|^{2} \leq t^{2} N$. Thus, $\mathbb{E} \max _{1 \leq j \leq m}\left|\mathbf{u}^{j}\right|^{2} \leq t^{2} N$, which implies that there is a big enough constant $c$ such that for every $m \geq c \frac{N t^{2}}{\varepsilon^{2}} \log N$ one has

$$
\left.\int_{\Omega^{m}} \sup _{\substack{\|f\|_{2} \leq 1, f \in X_{N}}}\left|\frac{1}{m} \sum_{j=1}^{m}\right| f\left(x^{j}\right)\right|^{2}-\int_{\Omega}|f(x)|^{2} \mu(d x) \mid \mu\left(d x^{1}\right) \ldots \mu\left(d x^{m}\right) \leq \varepsilon .
$$

Thus, there are points $\xi^{1}, \ldots, \xi^{m}$ such that

$$
\left.\sup _{\substack{\|f\|_{2} \leq 1, f \in X_{N}}}\left|\frac{1}{m} \sum_{j=1}^{m}\right| f\left(\xi^{j}\right)\right|^{2}-\int_{\Omega}|f(x)|^{2} \mu(d x) \mid \leq \varepsilon,
$$

which implies the $\mathcal{M}(m, 2, \varepsilon)$ theorem for the space $X_{N}$ while $m \geq c \frac{N t^{2}}{\varepsilon^{2}} \log N$. As we have already mentioned, the case $q=2$ is connected with random matrix theory, since in this case we approximate the covariance matrix of a random vector by its random sample. In particular, one can obtain the same result as above (see [155]) using the recent development of random matrix theory instead of Rudelson's Theorem 4.1 (see the discussion in Subsection 4.3 below). The further development of the sampling discretization problem in the case $q=2$ is connected with the deep result of A. Marcus, D.A. Spielman, and N. Srivastava from [99] and has already been discussed in the introduction and in Section 2.6. 
Returning to the problem of approximation by sample covariance matrix, the initial question of [64] about covariance matrices of uniform distributions on convex bodies was finally solved in terms of order in [1] (see also Chapter 10 in [25]) where the following two more general results were obtained.

Theorem 4.2. Let $\mathbf{u}^{1}, \ldots, \mathbf{u}^{m}$ be i.i.d. random vectors, distributed uniformly on a symmetric convex body in $\mathbb{R}^{N}$ and assume that the distribution is isotropic, i.e. $|y|^{2}=\mathbb{E}\left|\left\langle y, \mathbf{u}^{1}\right\rangle\right|^{2}$ for every $y \in \mathbb{R}^{N}$ (or vectors are distributed according to any isotropic logarithmically concave measure). Then for every $q \geq 2$ and for every $\varepsilon \in(0,1)$ there is a constant $C(\varepsilon, q)$ such that for any $m \geq C(\varepsilon, q) N^{\frac{q}{2}}$ one has $V_{q} \leq \varepsilon$ with probability at least $1-e^{-c_{p} \sqrt{N}}$ (where the constant $c_{q}>0$ depends only on $\left.q\right)$.

Theorem 4.3. Let $\mathbf{u}^{1}, \ldots, \mathbf{u}^{m}$ be i.i.d. random vectors, distributed uniformly on a symmetric convex body in $\mathbb{R}^{N}$ and assume that the distribution is isotropic, i.e. $|y|^{2}=\mathbb{E}\left|\left\langle y, \mathbf{u}^{1}\right\rangle\right|^{2}$ for every $y \in \mathbb{R}^{N}$ (or vectors are distributed according to any isotropic logarithmically concave measure). Then for every $q \in[1,2)$ and for every $\varepsilon \in(0,1)$ there is a constant $C(\varepsilon, q)$ such that for any $m \geq C(\varepsilon, q) N, m \leq e^{\sqrt{N}}$ one has $V_{q} \leq \varepsilon$ with probability at least $1-e^{-c \sqrt{N}}$ (where $c>0$ is an absolute constant).

Let now $\Omega$ be a symmetric convex set endowed with the normalized Lebesgue measure $\mu=\frac{1}{|\Omega|} \lambda_{\Omega}$. There is a linear isomorphism $T$ such that the random vector $\mathbf{u}:=T \mathbf{x}$ is isotropic, where $\mathbf{x}=\left(x_{1}, \ldots, x_{N}\right)$. Thus, we can apply Theorems 4.2 and 4.3 with this vector $\mathbf{u}$. In particular, for $q>2$, by Theorem 4.2 , there are $m \leq C(\varepsilon, q) N^{q / 2}$ points $\xi^{1}, \ldots, \xi^{m} \in \Omega$ such that

$$
\left.\left|\int_{\Omega}\right|\langle\mathbf{y}, T \mathbf{x}\rangle\right|^{q} \mu(d \mathbf{x})-\frac{1}{m} \sum_{j=1}^{m}\left|\left\langle\mathbf{y}, T \xi^{j}\right\rangle\right|^{q} \mid \leq \varepsilon\left(\int_{\Omega}|\langle\mathbf{y}, T \mathbf{x}\rangle|^{2} \mu(d \mathbf{x})\right)^{q / 2} .
$$

This bound implies $\mathcal{M}(m, q, \varepsilon)$ theorem for the space $X_{N}$ of all linear functionals on $\mathbb{R}^{N}$ with $m \leq C(\varepsilon, q) N^{q / 2}$ points for $q>2$, since the $L_{2}$ norm is bounded by the $L_{q}$ norm. Similarly, for $q \in[1,2)$ we can apply Theorem 4.3 and get the same bound (4.1) with $m \leq C(\varepsilon, q) N$ points $\xi^{1}, \ldots, \xi^{m}$. It is known that all the $L_{q}$ norms are equivalent on a space of all linear functions with respect to a logarithmically concave measure, thus

$$
\left(\int_{\Omega}|\langle y, T x\rangle|^{2} \mu(d x)\right)^{q / 2} \leq c(q) \int_{\Omega}|\langle y, T x\rangle|^{q} \mu(d x)
$$


for some number $c(q)$, that depends only on $q$. Thus, for $q \in[1,2)$ we get $\mathcal{M}(m, q, \varepsilon)$ theorem for the space of all linear functionals with $m \leq C(q) N$ points. An interesting open question is whether similar results are true for algebraic polynomials of fixed degree on convex domains $\Omega$.

In the case $q=2$ the further study (see [170], [53], [147], [105], [106], [163]) of the problem of approximation of the covariance matrix by a sample covariance matrix has continued for random vectors $\mathbf{u}$ under $2+\varepsilon$ moment assumption, i.e. it is assumed that

$$
\left(\mathbb{E}|\langle y, \mathbf{u}\rangle|^{p}\right)^{1 / p} \leq B\left(\mathbb{E}|\langle y, \mathbf{u}\rangle|^{2}\right)^{1 / 2} \quad \forall y \in \mathbb{R}^{N}
$$

for some $p>2$.

MM.2. We now discuss the case $q \neq 2$ and start with the results from [169] for random vectors under the assumption of higher order integrability of one-dimensional marginals, i.e. it is assumed that

$$
\left(\mathbb{E}|\langle y, \mathbf{u}\rangle|^{p}\right)^{1 / p} \leq B\left(\mathbb{E}|\langle y, \mathbf{u}\rangle|^{2}\right)^{1 / 2} \quad \forall y \in \mathbb{R}^{N}
$$

for some $p>\max \{q, 2\}$.

For such random vectors the following two results were obtained in [169].

Theorem 4.4. Let $q>2$ and let $\mathbf{u}$ be a random vector in $\mathbb{R}^{N}$ such that $|\mathbf{u}| \leq t \sqrt{N}$ a.s., $|y|^{2}=\mathbb{E}|\langle y, \mathbf{u}\rangle|^{2}$ for every $y \in \mathbb{R}^{N}$, and estimate (4.2) holds with $p=4 q$. Then for every $\varepsilon \in(0,1)$ and for every $\delta \in(0,1)$ there is a number $C:=C(t, B, q, \varepsilon, \delta)$ such that $V_{q} \leq \varepsilon$ with probability at least $1-\delta$ for every $m \geq C N^{\frac{q}{2}}$.

Theorem 4.5. Let $q \in[1,2)$ and let $\mathbf{u}$ be a random vector in $\mathbb{R}^{N}$ such that $|\mathbf{u}| \leq t \sqrt{N}$ a.s., $|y|^{2}=\mathbb{E}|\langle y, \mathbf{u}\rangle|^{2}$ for every $y \in \mathbb{R}^{N}$, and estimate (4.2) holds with $p \geq 4 q, p>4$. Then for every $\varepsilon \in(0,1)$ and for every $\delta \in(0,1)$ there is a number $C:=C(t, B, q, p, \varepsilon, \delta)$ such that $V_{q} \leq \varepsilon$ with probability at least $1-\delta$ for every $m \geq C N$.

The former of these two theorems is Theorem 1.1 in [169] and the latter is formulated in [169] in the remark after Theorem 1.1.

We now reformulate these theorems with regards to the general problem of sampling discretization for a subspace $X_{N}$ of $L_{q}(\Omega, \mu)$. 
Corollary 4.2. Let $q>2$ and let $X_{N}$ be an $N$-dimensional subspace of $L_{q}(\Omega, \mu)$. Assume that $\|f\|_{\infty} \leq t N^{\frac{1}{2}}\|f\|_{2}$ for every $f \in X_{N}$ and assume that

$$
\|f\|_{4 q} \leq B\|f\|_{2}
$$

for some constant $B>0$. Then for every $\varepsilon \in(0,1)$ there is a constant $C:=C(t, B, q, \varepsilon)$ such that $\mathcal{M}(m, q, \varepsilon)$ theorem holds for every $m \geq C N^{\frac{q}{2}}$.

Corollary 4.3. Let $q \in[1,2)$ and let $X_{N}$ be an $N$-dimensional subspace of $L_{q}(\Omega, \mu)$. Assume that $\|f\|_{\infty} \leq t N^{\frac{1}{2}}\|f\|_{2}$ for every $f \in X_{N}$ and assume that

$$
\|f\|_{p} \leq B\|f\|_{q}
$$

for some $p \geq 4 q, p>4$, and for some $B>0$. Then for every $\varepsilon \in(0,1)$ there is a constant $C:=C(t, B, q, p, \varepsilon)$ such that $\mathcal{M}(m, q, \varepsilon)$ theorem holds for every $m \geq C N$.

For example, conditions (4.3) and (4.4) are true for the space of all polynomials of a fixed degree on a convex domain $\Omega$ endowed with the normalized Lebesgue measure.

In particular, Corollary 4.3 implies that, with the extra assumption (4.4), one does not need the logarithmic oversampling. Therefore, Corollary 4.3 allows us to slightly improve bound (2.12) from $\mathbf{D} .16$ by imposing a rather strict assumption (4.4).

Both corollaries follow from Theorems 4.4 and 4.5 by consideration of the random vector $\mathbf{u}(x):=\left(u_{1}(x), \ldots, u_{N}(x)\right)$, where $u_{1}, \ldots, u_{N}$ is an orthonormal in $L_{2}(\Omega, \mu)$ basis in $X_{N}$. Indeed, for any $f \in X_{N}$ there is $\mathbf{y}=\left(y_{1}, \ldots, y_{N}\right)$ such that $f=\sum_{j=1}^{N} y_{j} u_{j}$ and

$$
\|f\|_{p}^{p}=\int_{\Omega}|f(x)|^{p} \mu(d x)=\int_{\Omega}\left|\sum_{j=1}^{N} y_{j} u_{j}(x)\right|^{p} \mu(d x)=\mathbb{E}|\langle\mathbf{y}, \mathbf{u}\rangle|^{p} .
$$

Thus, for $q \in[1,2)$ by Theorem 4.5 for every $\varepsilon>0$ there is a positive number $C:=C(t, B, q, \varepsilon)$ such that for any $m \geq C N$ there are points $\xi^{1}, \ldots, \xi^{m}$ for which

$$
\left.\sup _{\substack{\|f\|_{2} \leq 1,1 \\ f \in X_{N}}}\left|\frac{1}{m} \sum_{j=1}^{m}\right| f\left(\xi^{j}\right)\right|^{q}-\|f\|_{q}^{q} \mid \leq \frac{1}{B^{q}} \varepsilon .
$$


Since $p>2$ one has $\|f\|_{2}^{q} \leq\|f\|_{p}^{q} \leq B^{q}\|f\|_{q}^{q}$ and $X_{N} \in \mathcal{M}(m, q, \varepsilon)$.

For $q>2$ by Theorem 4.4 for every $\varepsilon>0$ there is a positive number $C:=C(t, B, q, \varepsilon)$ such that for any $m \geq C N^{\frac{q}{2}}$ there are points $\xi^{1}, \ldots, \xi^{m}$ for which

$$
\left.\sup _{\substack{\|f\|_{2} \leq 1, f \in X_{N}}}\left|\frac{1}{m} \sum_{j=1}^{m}\right| f\left(\xi^{j}\right)\right|^{q}-\|f\|_{q}^{q} \mid \leq \varepsilon .
$$

Since $q>2$ one has $\|f\|_{2}^{q} \leq\|f\|_{q}^{q}$ and $X_{N} \in \mathcal{M}(m, q, \varepsilon)$.

We point out that using a different type of approximation method one could improve the bound for the number of points in Theorem 4.4 (see the recent paper [103]).

MM.3. The last result of this subsection is due to O. Guedon and M. Rudelson (see [52, Theorem 3]).

Let $K$ be a symmetric convex body. Then $\|\cdot\|_{K}$ denote the norm in which $K$ is the unit ball, i.e. $\|z\|_{K}:=\inf \left\{s \geq 0: s^{-1} z \in K\right\}$. Let $p \geq 2$. We recall that the symmetric convex body $K$ in $\mathbb{R}^{N}$ is called $p$-convex with constant $\eta$ if

$$
\left\|\frac{z_{1}+z_{2}}{2}\right\|_{K} \leq 1-\eta\left\|z_{1}-z_{2}\right\|_{K}^{p}
$$

for all vectors $z_{1}, z_{2} \in K$.

Theorem 4.6. Let $K \subset \mathbb{R}^{N}$ be a symmetric convex body of radius $D$. Assume that $K$ is $p$-convex with constant $\eta$ for some $p \geq 2$. Assume that $q \geq p$. Then for any random vector $\mathbf{u}$ one has

$$
\mathbb{E} V_{q}(K) \leq A^{2}+A \sqrt{B}
$$

where

$$
A:=C^{q} \eta^{q} \frac{(\log m)^{1-1 / p}}{\sqrt{m}} D^{q / 2}\left(\mathbb{E} \max _{1 \leq j \leq m}\left|\mathbf{u}^{j}\right|^{q}\right)^{1 / 2}, \quad B:=\sup _{y \in K} \mathbb{E}|\langle y, \mathbf{u}\rangle|^{q} .
$$

This theorem implies the following result for the general problem of sampling discretization.

Corollary 4.4. Let $q \geq 2$ and let $X_{N}$ be an $N$-dimensional subspace of $L_{q}(\Omega, \mu)$ such that

$$
\|f\|_{\infty} \leq t \sqrt{N}\|f\|_{2} \quad \forall f \in X_{N} .
$$

Then for every $\varepsilon \in(0,1)$ there is a number $C:=C(t, q, \varepsilon)$ such that for each $m \geq C N^{\frac{q}{2}}[\log N]^{2-\frac{2}{q}}$ one has $X_{N} \in \mathcal{M}(m, q, \varepsilon)$. 
Indeed, we take $K=B_{q}:=\left\{f \in X_{N}:\|f\|_{q} \leq 1\right\}$. It is known that $B_{q}$ is $q$-convex with some constant $\eta=\eta(q)$ for $q \geq 2$. We again consider the random vector $\mathbf{u}:=\left(u_{1}(x), \ldots, u_{N}(x)\right)$, where $u_{1}, \ldots, u_{N}$ is an $L_{2}(\Omega, \mu)$ orthonormal basis in $X_{N}$, and again $|\mathbf{u}| \leq t \sqrt{N}$. Moreover, $\|f\|_{2} \leq\|f\|_{q}$, i.e. $D=1$. Thus,

$$
A \leq C(q) t^{q / 2} \frac{(\log m)^{1-1 / q}}{\sqrt{m}}(\sqrt{N})^{q / 2}, \quad B=1 .
$$

The discretization result now follows by the standard argument when we take $m \geq C(t, q, \varepsilon) N^{\frac{q}{2}}[\log N]^{2-\frac{2}{q}}$ for large enough constant $C(t, q, \varepsilon)$ such that $A \leq \frac{1}{4} \varepsilon$.

Lewis's change of density theorem (see [134, Theorem 2.1]), which we discuss further, combined with Corollary 4.4, gives the following statement about weighted sampling discretization.

Corollary 4.5. Let $q \geq 2$ and let $X_{N}$ be an $N$-dimensional subspace of $L_{q}(\Omega, \mu) \cap C(\Omega)$. Then for every $\varepsilon \in(0,1)$ there is a number $C:=C(q, \varepsilon)$ such that for each $m \geq C N^{\frac{q}{2}}[\log N]^{2-\frac{2}{q}}$ one has $X_{N} \in \mathcal{M}^{w}(m, q, \varepsilon)$.

Actually, one always has $X_{N} \in \mathcal{M}^{w}(m, q, \varepsilon)$ for each $m \geq C N^{\frac{q}{2}}[\log N]$. For example, this follows from the results on the embedding problem from Subsection 4.2 or from the slightly improved version of Theorem 4.6 from [86] (see Corollaries 4.4 and 4.5 and Remark 4.6 there).

\subsection{Embedding of finite-dimensional subspaces of $L_{q}$ into $\ell_{q}^{m}$}

Let $q \in[1, \infty)$ and $\varepsilon>0$ be fixed. The main problem of this subsection is to understand what is the smallest possible integer $m:=m_{q}(\varepsilon, N)$ such that for every $N$-dimensional subspace $X_{N}$ of $L_{q}([0,1]), 1 \leq q<\infty$ there is an $N$-dimensional subspace $Y_{N}$ of $\ell_{q}^{m}$ with $d\left(X_{N}, Y_{N}\right) \leq 1+\varepsilon$. Here $\ell_{q}^{m}$ is $\mathbb{R}^{m}$ endowed with the norm $\|y\|_{q}:=\left(\sum_{j=1}^{m}\left|y_{j}\right|^{q}\right)^{1 / q}$ and $d\left(X_{N}, Y_{N}\right)$ is the Banach-Mazur distance between $X_{N}$ and $Y_{N}$, which is the infimum of $\|T\| \cdot\left\|T^{-1}\right\|$ over all linear isomorphisms $T$ between $X_{N}$ and $Y_{N}$, where $\|\cdot\|$ is an operator norm (actually, the logarithm of $d$ is a distance).

This important and long standing problem has been extensively studied from the early 1980-s to the present by many mathematicians (see [19], [132], 
[133], [149], [150], [148], [134], [175] and citations therein). There is also a very nice expository paper by W.B. Johnson and G. Schechtman [60] where one could find a profound discussion of the problem along with some historical comments and more literature on the subject.

The main goal of this subsection is to present main ideas and methods of the theory behind the stated problem which can also be applied for various other problems. The important connection of this problem with the discretization problem from Section 2 is through the empirical method, which proposes to study the problem of embedding through discretization. We point out that this part of our paper is based on the above mentioned article [60] and two books' sections [148, Section 16.8] and [92, Section 15.5].

Em.1. First of all we formulate the known bounds for $m_{q}(\varepsilon, N)$. For $q>2$ one has $m_{q}(\varepsilon, N) \leq C(q, \varepsilon) N^{\frac{q}{2}} \log N$ (see [19]). For $q \in(1,2)$ one has $m_{q}(\varepsilon, N) \leq C(\varepsilon) N[\log N][\log \log N]^{2}$ (see [150]). For $q=1$ one has $m_{1}(\varepsilon, N) \leq C(\varepsilon) N \log N$ (see [149]). The problem when $q \in(0,1)$ has also been studied (see [134] and [175]) and the known bound is the same as for $q \in(1,2)$ (see $[175])$.

Em.2. We now discuss the main technique, which was initiated in [132], then developed in [19] and [149] and since then has been often applied in the study of the embedding problem. We point out that presented in this paper ideas do not provide the above best known results on their own and one needs some other technical steps to obtain the best known bounds for $m_{q}(\varepsilon, N)$.

First of all, one can always assume that the subspace $X_{N}$ of $L_{q}([0,1])$ is at a distance less than $1+\varepsilon$ from some $N$-dimensional subspace $X_{N}^{0}$ of $L_{q}\left(\Omega_{M}, \mu\right)$ for some large number $M$, where $\Omega_{M}=\left\{x_{1}, \ldots, x_{M}\right\}$ is a discrete point set with cardinality $M$ and $\mu$ is some probability measure on $\Omega_{M}$. We will write $\mu_{j}$ in place of $\mu\left(\left\{x_{j}\right\}\right)$. The first idea is to find a good replacement for the measure $\mu$, i.e. to find a measure $\nu_{j}=\varrho\left(x_{j}\right) \mu_{j}$, such that the subspace $\left(X_{N}^{0},\|\cdot\|_{L_{q}\left(\Omega_{M}, \mu\right)}\right)$ is isometric to some subspace $\left(Y_{N}^{0},\|\cdot\|_{L_{q}\left(\Omega_{M}, \nu\right)}\right)$ and this new subspace $Y_{N}^{0}$ possesses some good properties. This can be done via the Lewis change of density theorem (see [93]). We formulate this theorem as it was stated in [134], where the result of Lewis was extended to the case $q \in(0,1)$.

Theorem 4.7. Let $X_{N}$ be an $N$-dimensional subspace of $L_{q}(\Omega, \mu)$, where 
$0<q<\infty$. Then there is a basis $f_{1}, \ldots, f_{N}$ in $X_{N}$ such that

$$
\int_{\Omega}\left(\sum_{k=1}^{N} f_{k}^{2}\right)^{\frac{q-2}{2}} f_{i} f_{j} d \mu=\delta_{i, j} \quad \forall i, j \in\{1, \ldots, N\} .
$$

In particular, there is a probability measure $\nu$ on $\Omega$ and a space $Y_{N}$ in $L_{q}(\Omega, \nu)$ which is isometric to $X_{N}$ and which has a basis $u_{1}, \ldots, u_{N}$ that is orthonormal in $L_{2}(\Omega, \nu)$ such that $\sum_{k=1}^{N} u_{k}^{2}(x)=N \quad \forall x \in \Omega$.

Em.3. Lewis's theorem implies that there is a new probability measure $\nu=\varrho \mu$ on $\Omega_{M}$ such that $\left(X_{N}^{0},\|\cdot\|_{L_{q}\left(\Omega_{M}, \mu\right)}\right)$ is isometric to the subspace

$$
Y_{N}^{0}:=\left\{f^{\prime}: \Omega_{M} \rightarrow \mathbb{R}: f^{\prime}\left(x_{j}\right)=\varrho\left(x_{j}\right)^{-1 / q} f\left(x_{j}\right), f \in X_{N}^{0}\right\}
$$

of $L_{q}\left(\Omega_{M}, \nu\right)$ and there is a basis $u_{1}, \ldots, u_{N}$ of $Y_{N}^{0}$ that is orthonormal in $L_{2}\left(\Omega_{M}, \nu\right)$ such that $\sum_{k=1}^{N} u_{k}^{2}(x)=N$ for each $x \in \Omega_{M}$. Moreover, one can split the atoms $\nu_{i}$, which are greater than $\frac{2}{M}$, into $\left[\nu_{i} \frac{M}{2}\right]+1$ equal pieces. The cardinality $M^{\prime}$ of the new set $\Omega_{M^{\prime}}^{\prime}=\left\{x_{1}^{\prime}, \ldots, x_{M^{\prime}}^{\prime}\right\}$ after the splitting is not greater than $\frac{3 M}{2}$. Let $\mu^{\prime}$ be the new measure on $\Omega_{M^{\prime}}^{\prime}$ which appears from $\nu$ after the splitting of atoms, $\mu_{j}^{\prime} \leq \frac{2}{M}$. Let $X_{N}^{\prime}$ be a subset of $L_{q}\left(\Omega_{M^{\prime}}^{\prime}, \mu^{\prime}\right)$ which appears from $Y_{N}^{0}$ after the splitting of atoms.

Em.4. The next idea is to "split" an $L_{q}\left(\Omega_{M^{\prime}}^{\prime}, \mu^{\prime}\right)$ norm on $X_{N}^{\prime}$ into two almost equal parts, i.e. one wants to find a splitting of the set $\Omega_{M^{\prime}}^{\prime}$ into two disjoint parts $W_{1}$ and $W_{2}$ such that

$$
\left(\frac{1}{2}-\varepsilon_{N, M^{\prime}}\right) \sum_{j=1}^{M^{\prime}} \mu_{j}^{\prime}\left|f^{\prime}\left(x_{j}^{\prime}\right)\right|^{q} \leq \sum_{x_{j} \in W_{i}} \mu_{j}^{\prime}\left|f^{\prime}\left(x_{j}^{\prime}\right)\right|^{q} \leq\left(\frac{1}{2}+\varepsilon_{N, M^{\prime}}\right) \sum_{j=1}^{M^{\prime}} \mu_{j}^{\prime}\left|f^{\prime}\left(x_{j}^{\prime}\right)\right|^{q}
$$

for each $f^{\prime} \in X_{N}^{\prime}$ and $i \in\{1,2\}$. We point out that exactly this type of splitting is one of the main results of A. Marcus, D.A. Spielman, and N. Srivastava from paper [99].

Clearly, one of the sets $W_{1}$ or $W_{2}$ is of cardinality not greater than $\frac{1}{2} M^{\prime}$, i.e. of cardinality bounded by $\frac{3}{4} M$ and exactly this set we denote $\Omega_{M_{1}}^{1}$, $M_{1} \leq \frac{3}{4} M$. If we return to the initial measure $\mu$ and the subspace $X_{N}$, the above bound actually reads as

$$
\left(\frac{1}{2}-\varepsilon_{N, M^{\prime}}\right) \sum_{j=1}^{M} \mu_{j}\left|f\left(x_{j}\right)\right|^{q} \leq \sum_{y_{j} \in \Omega_{M_{1}}^{1}} \mu_{j}^{1}\left|f\left(y_{j}\right)\right|^{q} \leq\left(\frac{1}{2}+\varepsilon_{N, M^{\prime}}\right) \sum_{j=1}^{M} \mu_{j}\left|f\left(x_{j}\right)\right|^{q}
$$


for all $f \in X_{N}$, where $\left\{\mu_{j}^{1}\right\}$ are new weights (new measure) on the new domain $\Omega_{M_{1}}^{1}$ with cardinality $M_{1} \leq \lambda M, \lambda=\frac{3}{4}$. Then one iterates the described procedure and, while $\prod_{k=0}^{k_{0}}\left(\frac{1+\varepsilon_{N, \lambda} k_{M}}{1-\varepsilon_{N, \lambda} k_{M}}\right)^{1 / q} \leq 1+\varepsilon$, the initial subspace $X_{N}$ is at a distance at most $1+\varepsilon$ from an $N$-dimensional subspace in $\ell_{q}^{m}$ with $m \leq \lambda^{-k_{0}-1} M$.

The above iteration procedure was applied to the embedding problem in [149] but a similar idea of iteration has also appeared earlier in the work of Bourgain, Lindenstrauss, and Milman [19] and in the work of Lunin [97] where he studied the problem of finding a submatrix with the smallest possible norm in a given matrix (see more on this in Section 3).

Em.5. The first bound in Em.4. is equivalent to the bound

$$
\left.\left|\sum_{x_{j} \in W_{1}} \mu_{j}^{\prime}\right| f^{\prime}\left(x_{j}^{\prime}\right)\right|^{q}-\left.\sum_{x_{j} \in \Omega_{M^{\prime}}^{\prime} \backslash W_{1}} \mu_{j}^{\prime}\left|f^{\prime}\left(x_{j}^{\prime}\right)\right|^{q}\left|\leq 2 \varepsilon_{N, M^{\prime}} \sum_{j=1}^{M^{\prime}} \mu_{j}^{\prime}\right| f^{\prime}\left(x_{j}^{\prime}\right)\right|^{q} \quad \forall f^{\prime} \in X_{N}^{\prime} .
$$

This inequality means that there is a choice of signs $\left\{\varepsilon_{1}^{0}, \ldots, \varepsilon_{M^{\prime}}^{0}\right\}$ such that

$$
\left.\left.\left|\sum_{j=1}^{M^{\prime}} \varepsilon_{j}^{0} \mu_{j}^{\prime}\right| f\left(x_{j}^{\prime}\right)\right|^{q}\left|\leq 2 \varepsilon_{N, M^{\prime}} \sum_{j=1}^{M^{\prime}} \mu_{j}^{\prime}\right| f^{\prime}\left(x_{j}^{\prime}\right)\right|^{q} \quad \forall f^{\prime} \in X_{N}^{\prime}
$$

or, equivalently,

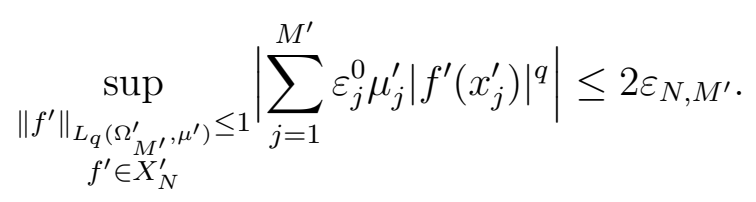

Clearly, there is a choice of signs $\left\{\varepsilon_{1}^{0}, \ldots, \varepsilon_{M^{\prime}}^{0}\right\}$ such that

$$
\left.\sup _{\left\|f^{\prime}\right\|_{L_{q}\left(\Omega_{M}^{\prime}, \mu^{\prime}\right)} \leq 1}\left|\sum_{j=1}^{M^{\prime} \in X_{N}^{\prime}} \varepsilon_{j}^{0} \mu_{j}^{\prime}\right| f^{\prime}\left(x_{j}^{\prime}\right)\right|^{q}\left|\leq \mathbb{E} \sup _{\substack{\left\|f^{\prime}\right\|_{L_{q}\left(\Omega^{\prime}, M^{\prime}, \mu^{\prime}\right)} \leq 1 \\ f^{\prime} \in X_{N}^{\prime}}}\right| \sum_{j=1}^{M^{\prime}} \varepsilon_{j} \mu_{j}^{\prime}\left|f^{\prime}\left(x_{j}^{\prime}\right)\right|^{q} \mid,
$$

where $\mathbb{E}$ stands for the average over all possible choice of signs $\left\{\varepsilon_{1}, \ldots, \varepsilon_{M^{\prime}}\right\}$. Thus, one interested in the bounds for the expectation

$$
\left.\mathbb{E} \sup _{\substack{\left\|f^{\prime}\right\|_{L_{q}\left(\Omega^{\prime} M^{\prime}, \mu^{\prime}\right)} \leq 1 \\ f^{\prime} \in X_{N}^{\prime}}}\left|\sum_{j=1}^{M^{\prime}} \varepsilon_{j} \mu_{j}^{\prime}\right| f^{\prime}\left(x_{j}^{\prime}\right)\right|^{q} \mid
$$


and the embedding problem reduces to finding good bounds for the expectation of the supremum of a random process over a convex set. These bounds are similar to the one discussed in Subsection 4.1 and can be studied by the same techniques (e.g. by the chaining technique).

In the case $p \in[1,2)$ the bounds for the expectation above have been obtained by Talagrand (see [92, Theorem 15.12] and [148, Theorem 16.8.2] or [149] and [150]).

We point out that in the case $p>2$ the paper [19] uses a different approach.

Em.6. As it was by G. Schechtman brought to our attention that these methods of proofs may actually provide the same bounds for the number of points in the corresponding problems of sampling discretization with weights.

\subsection{Sparse approximation}

We have explained in Section 1 (see (1.11)) how sampling discretization of the $L_{2}$ norm is connected with the $m$-term approximation of the identity matrix $I$ with respect to the system $\{G(x)\}_{x \in \Omega}$ of rank one matrices. Namely, for a set of points $\xi^{k} \in \Omega, k=1, \ldots, m$, and $f=\sum_{i=1}^{N} b_{i} u_{i}$ we have

$$
\frac{1}{m} \sum_{k=1}^{m} f\left(\xi^{k}\right)^{2}-\int_{\Omega} f(x)^{2} d \mu=\mathbf{b}^{T}\left(\frac{1}{m} \sum_{k=1}^{m} G\left(\xi^{k}\right)-I\right) \mathbf{b}
$$

where $\mathbf{b}=\left(b_{1}, \ldots, b_{N}\right)^{T}$ is the column vector. Therefore,

$$
\left|\frac{1}{m} \sum_{k=1}^{m} f\left(\xi^{k}\right)^{2}-\int_{\Omega} f(x)^{2} d \mu\right| \leq\left\|\frac{1}{m} \sum_{k=1}^{m} G\left(\xi^{k}\right)-I\right\|\|\mathbf{b}\|_{2}^{2},
$$

where $\|\cdot\|$ is the operator norm (spectral norm) of a matrix. Then the following deep result (see [165], Theorem 1.1) on random matrices can be used.

Theorem 4.8. Consider a finite sequence $\left\{T_{k}\right\}_{k=1}^{m}$ of independent, random, self-adjoint matrices with dimension $N$. Assume that each random matrix is positive semi-definite and satisfies

$$
\lambda_{\max }\left(T_{k}\right) \leq R \quad \text { almost surely }
$$


Note that $\lambda_{\max }$ and $\lambda_{\min }$ denote the maximal and the minimal eigenvalues. Define

$$
s_{\min }:=\lambda_{\min }\left(\sum_{k=1}^{m} \mathbb{E}\left(T_{k}\right)\right) \quad \text { and } \quad s_{\max }:=\lambda_{\max }\left(\sum_{k=1}^{m} \mathbb{E}\left(T_{k}\right)\right) .
$$

Then

$$
\mathbb{P}\left\{\lambda_{\min }\left(\sum_{k=1}^{m} T_{k}\right) \leq(1-\eta) s_{\min }\right\} \leq N\left(\frac{e^{-\eta}}{(1-\eta)^{1-\eta}}\right)^{s_{\min } / R}
$$

for $\eta \in[0,1)$ and for $\eta \geq 0$

$$
\mathbb{P}\left\{\lambda_{\max }\left(\sum_{k=1}^{m} T_{k}\right) \geq(1+\eta) s_{\max }\right\} \leq N\left(\frac{e^{\eta}}{(1+\eta)^{1+\eta}}\right)^{s_{\max } / R} .
$$

It is shown in [155] that Theorem 4.8 implies the following sampling discretization result: Let $\left\{u_{i}\right\}_{i=1}^{N}$ be an orthonormal system, satisfying Condition E. Then

$$
X_{N}=\operatorname{span}\left\{u_{1}, \ldots, u_{N}\right\} \in \mathcal{M}(m, 2, \varepsilon) \quad \text { provided } \quad m \geq C \frac{t^{2}}{\varepsilon^{2}} N \log N .
$$

We only formulated one result - Theorem 4.8 - from the theory of random matrices which is useful in sampling discretization. We refer the reader for other results in this direction to the papers [130], [165], [117], [127], [51], and [104]. Also, we mention a very recent paper [107] where results from the theory of random matrices were applied to sampling discretization and recovery.

Inequality (4.5), shows that the Marcinkiewicz-type discretization theorem in $L_{2}$ is closely related to approximation of the identity matrix $I$ by an $m$-term approximant of the form $\frac{1}{m} \sum_{k=1}^{m} G\left(\xi^{k}\right)$ in the operator norm from $\ell_{2}^{N}$ to $\ell_{2}^{N}$ (spectral norm). Therefore, we can consider the following sparse approximation problem: Assume that the system $\left\{u_{i}(x)\right\}_{i=1}^{N}$ satisfies Condition E and consider the dictionary

$$
\mathcal{D}^{u}:=\left\{g_{x}\right\}_{x \in \Omega}, \quad g_{x}:=G(x)\left(N t^{2}\right)^{-1}, \quad G(x):=\left[u_{i}(x) u_{j}(x)\right]_{i, j=1}^{N} .
$$

Let the Hilbert space $H$ be the closure in the Fröbenius norm of the space $\operatorname{span}\left\{g_{x}, x \in \Omega\right\}$ with the inner product generated by the Fröbenius norm: 
for $A=\left[a_{i, j}\right]_{i, j=1}^{N}$ and $B=\left[b_{i, j}\right]_{i, j=1}^{N}$

$$
\langle A, B\rangle=\sum_{i, j=1}^{N} a_{i, j} b_{i, j}
$$

in case of real matrices (with standard modification in case of complex matrices).

By known results from greedy approximation (see, for instance, [153], p.90, Theorem 2.15) we obtain that, for any $m \in \mathbb{N}$, we can constructively find (by the Relaxed Greedy Algorithm) points $\xi^{1}, \ldots, \xi^{m}$ such that

$$
\left\|\frac{1}{m} \sum_{k=1}^{m} G\left(\xi^{k}\right)-I\right\|_{F} \leq 2 N t^{2} m^{-1 / 2} .
$$

Taking into account the inequality $\|A\| \leq\|A\|_{F}$ and (4.5) we get from (4.6) the following proposition.

Proposition 4.1. Let $\left\{u_{i}\right\}_{i=1}^{N}$ be an orthonormal system, satisfying Condition E. Then there exists a constructive set $\left\{\xi^{j}\right\}_{j=1}^{m} \subset \Omega$ with $m \leq C(t) N^{2}$ such that for any $f=\sum_{i=1}^{N} c_{i} u_{i}$ we have

$$
\frac{1}{2}\|f\|_{2}^{2} \leq \frac{1}{m} \sum_{j=1}^{m} f\left(\xi^{j}\right)^{2} \leq \frac{3}{2}\|f\|_{2}^{2} .
$$

We now comment on a breakthrough result by J. Batson, D.A. Spielman, and N. Srivastava [5] on the weighted sampling discretization. We formulate their result in our notation. Let as above $\Omega_{M}=\left\{x^{j}\right\}_{j=1}^{M}$ be a discrete set with the probability measure $\mu\left(x^{j}\right)=1 / M, j=1, \ldots, M$. Assume that $\left\{u_{i}(x)\right\}_{i=1}^{N}$ is a real orthonormal on $\Omega_{M}$ system. Then for any number $d>1$ there exist a set of weights $w_{j} \geq 0$ such that $\left|\left\{j: w_{j} \neq 0\right\}\right| \leq d N$ so that for any $f \in \operatorname{span}\left\{u_{1}, \ldots, u_{N}\right\}$ we have

$$
\|f\|_{2}^{2} \leq \sum_{j=1}^{M} w_{j} f\left(x^{j}\right)^{2} \leq \frac{d+1+2 \sqrt{d}}{d+1-2 \sqrt{d}}\|f\|_{2}^{2} .
$$

The proof of this result is based on a delicate study of the $m$-term approximation of the identity matrix $I$ with respect to the system $\mathcal{D}:=\{G(x)\}_{x \in \Omega}$, $G(x):=\left[u_{i}(x) u_{j}(x)\right]_{i, j=1}^{N}$ in the spectral norm. The authors of [5] control the 
change of the maximal and minimal eigenvalues of a matrix, when they add a rank one matrix of the form $w G(x)$. Their proof provides an algorithm for construction of the weights $\left\{w_{j}\right\}$. In particular, this implies that

$$
X_{N}\left(\Omega_{M}\right) \in \mathcal{M}^{w}(m, 2, \varepsilon) \quad \text { provided } \quad m \geq C N \varepsilon^{-2}
$$

with large enough $C$.

\subsection{Supervised learning theory}

In Section 1 we have discussed one of the settings of learning theory. In this subsection we present some of the well known results from supervised learning theory, comment on important techniques, and discuss connection with the sampling discretization. The chaining technique and concentration of measure inequalities are the foundation blocks of both learning theory and sampling discretization. We begin with a detailed discussion of the interplay between the use of expectation and the probability distribution function in the error analysis. For the reader's convenience we begin our discussion with an application of probabilistic methods in numerical integration. We recall the classical Monte Carlo method from numerical integration. Let $\Omega$ be a bounded domain of $\mathbb{R}^{d}$. Consider a real function $f \in L_{2}(\Omega):=L_{2}(\Omega, \mu)$

with respect to a probability measure $\mu$. Define $\Omega^{m}:=\Omega \times \cdots \times \Omega$ and $\mu^{m}:=\mu \times \cdots \times \mu$. For $\mathbf{x}^{j} \in \Omega$ denote $\mathbf{z}:=\left(\mathbf{x}^{1}, \ldots, \mathbf{x}^{m}\right) \in \Omega^{m}$ and for $g \in L_{1}\left(\Omega^{m}, \mu^{m}\right)$

$$
\mathbb{E}(g):=\int_{\Omega^{m}} g(\mathbf{z}) d \mu^{m}
$$

Then it is known that for $f \in L_{2}(\Omega, \mu)$ we have

$$
\mathbb{E}\left(\left(\int_{\Omega} f d \mu-\frac{1}{m} \sum_{j=1}^{m} f\left(\mathbf{x}^{j}\right)\right)^{2}\right) \leq \frac{\|f\|_{2}^{2}}{m} .
$$

In particular, inequality (4.7) guarantees existence of a cubature formula $Q_{m}(\cdot, \xi)$ with $\xi:=\left\{\xi^{j}\right\}_{j=1}^{m}$ such that

$$
\left|\int_{\Omega} f d \mu-Q_{m}(f, \xi)\right| \leq m^{-1 / 2}\|f\|_{2} .
$$

Certainly, the existence of a good cubature formula for an individual function is a simple fact. For a function $f$ that is continuous on $\Omega$ we can always 
find a point $\xi^{1}$ and a weight $\lambda_{1}$ such that $\int_{\Omega} f d \mu=\lambda_{1} f\left(\xi^{1}\right)$. However, the above probabilistic argument allows us to guarantee a bound similar to (4.8) with high probability. This observation, in turn, allows us to find a cubature formula, which is simultaneously good for several functions. However, the use of expectation does not provide a good enough bound on the probability to guarantee a tight error bound like (4.8) for many functions. The concentration of measure inequalities, which we formulate momentarily, provide a very good bound on the probability under some extra assumptions on $f$. Under the condition $\|f\|_{\infty} \leq M$ the Hoeffding inequality (see, for instance, [153], p.197) gives

$$
\mu^{m}\left\{\mathbf{z}:\left|\int_{\Omega} f d \mu-\frac{1}{m} \sum_{j=1}^{m} f\left(\mathbf{x}^{j}\right)\right| \geq \eta\right\} \leq 2 \exp \left(-\frac{m \eta^{2}}{8 M^{2}}\right) .
$$

Bernstein's inequality (see, for instance, [153], p.198) gives the following bound under conditions $\|f\|_{\infty} \leq M_{\infty}$ and $\|f\|_{2} \leq M_{2}$

$$
\mu^{m}\left\{\mathbf{z}:\left|\int_{\Omega} f d \mu-\frac{1}{m} \sum_{j=1}^{m} f\left(\mathbf{x}^{j}\right)\right| \geq \eta\right\} \leq 2 \exp \left(-\frac{m \eta^{2}}{2\left(M_{2}^{2}+2 M_{\infty} \eta / 3\right)}\right) .
$$

The above inequalities (4.9) and (4.10) can be used directly for proving the existence of good cubature formulas for function classes containing a finite number of elements. Denote by $|\mathbf{W}|$ the cardinality of a set $\mathbf{W}$. Assume that for all $f \in \mathbf{W}$ we have $\|f\|_{\infty} \leq M$. Then, the Hoeffding inequality (4.9) gives

$$
\mu^{m}\left\{\mathbf{z}: \sup _{f \in \mathbf{W}}\left|\int_{\Omega} f d \mu-\frac{1}{m} \sum_{j=1}^{m} f\left(\mathbf{x}^{j}\right)\right| \leq \eta\right\} \geq 1-2|\mathbf{W}| \exp \left(-\frac{m \eta^{2}}{8 M^{2}}\right) .
$$

Thus, when the right hand side of (4.11) is positive, inequality (4.11) guarantees the existence of a good cubature formula for the whole class $\mathbf{W}$. However, it is clear from relation (4.11) that such an argument does not work for a class $\mathbf{W}$, which contains infinitely many functions. It is known that chaining techniques allow us to overcome this problem.

We now concentrate on results on the probability distribution function of the defect function introduced in Section 1. We use notation from Section 1. An important consideration in finding an optimal $f_{\mathbf{z}}$ is how to describe the class $\Theta$ of priors? In other words, what characteristics of $\Theta$ govern, say, 
the optimal rate of decay of $\mathbb{E}\left(\left\|f_{\rho}-f_{\mathbf{z}}\right\|_{L_{2}\left(\rho_{\Omega}\right)}^{2}\right)$ for $f_{\rho} \in \Theta$ ? Previous and recent works in statistics and learning theory (see, for instance, [37], [85], and [153], Ch.4) indicate that the compactness characteristics of $\Theta$ play a fundamental role. It is convenient for us to express compactness of $\Theta$ in terms of entropy numbers. We discuss the classical concept of entropy (see Subsection 2.2). We note that some other concepts of entropy, for instance, entropy with bracketing, prove to be useful in the theory of empirical processes and nonparametric statistics (see [167], [166]). There is a concept of $V C$ dimension that plays a fundamental role in the problem of pattern recognition and classification [166]. This concept is also useful in describing compactness characteristics of sets.

In this subsection we assume that the measure $\rho$ is concentrated on a set that is bounded with respect to $y$, i.e. the set $Z$ satisfies the condition $Z \subset \Omega \times[-M, M]$ (or a little weaker $|y| \leq M$ a.e. with respect to $\rho_{\Omega}$, i.e the $\rho_{\Omega}$-measure of those $\mathbf{x}$, for which there exists a $y$ such that $(\mathbf{x}, y) \in Z$ and $|y|>M$ is equal to zero) with some fixed $M$. Then it is clear that for $f_{\rho}$ we have $\left|f_{\rho}(\mathbf{x})\right| \leq M$ for all $\mathbf{x}$ (for almost all $\mathbf{x}$ ). Therefore, it is natural to assume that a class $\Theta$ of priors where $f_{\rho}$ belongs is embedded into the $\mathcal{C}(X)$-ball $\left(L_{\infty}\right.$-ball $)$ of radius $M$.

We begin with the case of $\mathcal{C}(\Omega)$, the space of functions continuous on a compact subset $\Omega$ of $\mathbb{R}^{d}$ with the norm

$$
\|f\|_{\infty}:=\sup _{\mathbf{x} \in \Omega}|f(\mathbf{x})|
$$

We use the abbreviated notation

$$
\varepsilon_{n}(\mathbf{F}):=\varepsilon_{n}(\mathbf{F}, \mathcal{C})
$$

The following well known theorem (see, for instance, [153], p.211) shows how compactness characteristics of $\mathbf{F}$ can be used in estimating the defect function.

Theorem 4.9. Let $\mathbf{F}$ be a compact subset of $\mathcal{C}(\Omega)$. Assume that there exists $M>0$ such that for all $f \in \mathbf{F}$ and for all $(\mathbf{x}, y) \in Z$ we have $|f(\mathbf{x})-y| \leq M$. Then, for all $\varepsilon>0$

$$
\rho^{m}\left\{\mathbf{z}: \sup _{f \in \mathbf{F}}\left|L_{\mathbf{z}}(f)\right| \leq \varepsilon\right\} \geq 1-2 N\left(\mathbf{F}, \frac{\varepsilon}{8 M}\right) \exp \left(-\frac{m \varepsilon^{2}}{8\left(\sigma^{2}+M^{2} \varepsilon / 6\right)}\right) .
$$


Here $\sigma^{2}:=\sup _{f \in \mathbf{F}} \sigma^{2}\left((f(\mathbf{x})-y)^{2}\right)$ and $\sigma^{2}(g)$ is the variance of a random variable $g$.

Remark 4.1. In general we cannot guarantee that the set

$\left\{\mathbf{z}: \sup _{f \in \mathbf{F}}\left|L_{\mathbf{z}}(f)\right| \geq \eta\right\}$ is $\rho^{m}$-measurable. In such a case the relation (4.12) and further relations of this type are understood in the sense of outer measure associated with the $\rho^{m}$. For instance, for (4.12) this means that there exists $\rho^{m}$-measurable set $G$ such that $\left\{\mathbf{z}: \sup _{f \in \mathbf{F}}\left|L_{\mathbf{z}}(f)\right| \geq \eta\right\} \subset G$ and (4.12) holds for $G$.

We note that Theorem 4.9 is related to the concept of the GlivenkoCantelli sample complexity of a class $\Phi$ with accuracy $\varepsilon$ and confidence $\delta$ :

$$
\begin{gathered}
S_{\Phi}(\varepsilon, \delta):=\min \{n: \quad \text { for all } m \geq n, \text { for all } \rho \\
\left.\rho^{m}\left\{\mathbf{z}=\left(z_{1}, \ldots, z_{m}\right): \sup _{\phi \in \Phi}\left|\int_{Z} \phi d \rho-\frac{1}{m} \sum_{i=1}^{m} \phi\left(z_{i}\right)\right| \geq \varepsilon\right\} \leq \delta\right\} .
\end{gathered}
$$

In order to see that, we define points $z_{i}:=\left(\mathbf{x}^{i}, y_{i}\right), i=1, \ldots, m$; functions $\phi(\mathbf{x}, y):=(f(\mathbf{x})-y)^{2}$; and, finally, the class $\Phi:=\left\{(f(\mathbf{x})-y)^{2}, f \in \mathbf{F}\right\}$. One can find a survey of results on the Glivenko-Cantelli sample complexity in [102] and find results and the corresponding historical remarks related to Theorem 4.9 in [54].

We now formulate two theorems, which provide somewhat more delicate estimates for the defect function (see [153], pp. 213-217). We assume that $\rho$ and $\mathbf{F}$ satisfy the following condition.

For all $f \in \mathbf{F}, \quad f: \Omega \rightarrow Y \quad$ and any $(\mathbf{x}, y) \in Z, \quad|f(\mathbf{x})-y| \leq M$.

The following Theorem 4.10 is from [84] (see also [153], Section 4.3.3, p.213). We point out that the proofs of both Theorems 4.10 and 4.11 are based on the chaining technique and on the concentration measure inequalities. Some of the ideas here are similar to the previously discussed ideas, which were used for analyzing Monte Carlo cubature formulas.

Theorem 4.10. Assume that $\rho, \mathbf{F}$ satisfy (4.13) and $\mathbf{F}$ is such that

$$
\sum_{n=1}^{\infty} n^{-1 / 2} \varepsilon_{n}(\mathbf{F})<\infty .
$$


Then for $m \eta^{2} \geq 1$ we have

$$
\rho^{m}\left\{\mathbf{z}: \sup _{f \in \mathbf{F}}\left|L_{\mathbf{z}}(f)\right| \geq \eta\right\} \leq C(M, \varepsilon(\mathbf{F})) \exp \left(-c(M) m \eta^{2}\right)
$$

with $C(M, \varepsilon(\mathbf{F}))$ that may depend on $M$ and $\varepsilon(\mathbf{F}):=\left\{\varepsilon_{n}(\mathbf{F}, \mathcal{C})\right\} ; c(M)$ may depend only on $M$.

Theorem 4.11. Assume that $\rho, \mathbf{F}$ satisfy (4.13) and $\mathbf{F}$ is such that

$$
\sum_{n=1}^{\infty} n^{-1 / 2} \varepsilon_{n}(\mathbf{F})=\infty .
$$

For $\eta>0$ define $J:=J(\eta / M)$ as the minimal $j$ satisfying $\varepsilon_{2^{j}} \leq \eta /(8 M)$ and

$$
S_{J}:=\sum_{j=1}^{J} 2^{(j+1) / 2} \varepsilon_{2^{j-1}} .
$$

Then for $m, \eta$ satisfying $m\left(\eta / S_{J}\right)^{2} \geq 480 M^{2}$ we have

$$
\rho^{m}\left\{\mathbf{z}: \sup _{f \in \mathbf{F}}\left|L_{\mathbf{z}}(f)\right| \geq \eta\right\} \leq C(M, \varepsilon(\mathbf{F})) \exp \left(-c(M) m\left(\eta / S_{J}\right)^{2}\right) .
$$

Corollary 4.6. Assume $\rho, \mathbf{F}$ satisfy (4.13) and $\varepsilon_{n}(\mathbf{F}) \leq D n^{-1 / 2}$. Then for $m$, $\eta$ satisfying $m(\eta /(1+\log (M / \eta)))^{2} \geq C_{1}(M, D)$ we have

$$
\rho^{m}\left\{\mathbf{z}: \sup _{f \in \mathbf{F}}\left|L_{\mathbf{z}}(f)\right| \geq \eta\right\} \leq C(M, D) \exp \left(-c(M, D) m(\eta /(1+\log (M / \eta)))^{2}\right) .
$$

Corollary 4.7. Assume $\rho, \mathbf{F}$ satisfy (4.13) and $\varepsilon_{n}(\mathbf{F}) \leq D n^{-r}, r \in(0,1 / 2)$. Then for $m, \eta$ satisfying $m \eta^{1 / r} \geq C_{1}(M, D, r)$ we have

$$
\rho^{m}\left\{\mathbf{z}: \sup _{f \in \mathbf{F}}\left|L_{\mathbf{z}}(f)\right| \geq \eta\right\} \leq C(M, D, r) \exp \left(-c(M, D, r) m \eta^{1 / r}\right) .
$$

We now demonstrate how the above results from learning theory can be used for sampling discretization of the $L_{2}$ norm. We use notation from Section 1. Condition (4.13) is satisfied with $M$ such that for all $f \in \mathbf{F}$ we have $\|f\|_{\infty} \leq M$. The argument from the Introduction shows that we can derive results on discretization of the $L_{2}$ norm directly from the corresponding results from learning theory. We assume that $\mathbf{F}$ satisfies the following condition:

$$
f \in \mathbf{F} \quad \Rightarrow \quad\|f\|_{\infty} \leq M .
$$

Theorem 4.10 implies the following result (see [158]). 
Theorem 4.12. Assume that $\mathbf{F}$ satisfies (4.14) and the condition

$$
\sum_{n=1}^{\infty} n^{-1 / 2} \varepsilon_{n}(\mathbf{F})<\infty .
$$

Then there exists a constant $K$ such that for any $m$ there is a set of points $\xi=\left\{\xi^{1}, \ldots, \xi^{m}\right\}$ such that

$$
e r_{m}\left(\mathbf{F}, L_{2}\right) \leq K m^{-1 / 2} .
$$

In particular, if

$$
\varepsilon_{n}(\mathbf{F}) \leq C_{1} n^{-r}, \quad r>1 / 2,
$$

then

$$
e r_{m}\left(\mathbf{F}, L_{2}\right) \leq C\left(r, C_{1}\right) m^{-1 / 2} .
$$

The reader can find further results in this direction in the recent paper [107].

Corollary 4.7 implies the following result (see [158]).

Theorem 4.13. Assume that $\mathbf{F}$ satisfies (4.14) and the condition

$$
\varepsilon_{n}(\mathbf{F}) \leq C_{1} n^{-r}, \quad r \in(0,1 / 2) .
$$

Then there exists a constant $C\left(r, C_{1}\right)$ such that for any $m$ there is a set of points $\xi=\left\{\xi^{1}, \ldots, \xi^{m}\right\}$ such that

$$
e r_{m}\left(\mathbf{F}, L_{2}\right) \leq C\left(r, C_{1}\right) m^{-r} .
$$

Acknowledgements. The authors are grateful to Feng Dai, Tino Ullrich, Mario Ullrich, and the referees for their useful comments and suggestions. The second author is a Young Russian Mathematics award winner and would like to thank its sponsors and jury.

The work was supported by the Russian Federation Government Grant №14.W03.31.0031.

\section{References}

[1] R. Adamczak, A. Litvak, A. Pajor, and N. Tomczak-Jaegermann, Quantitative estimates of the convergence of the empirical covariance matrix in log-concave ensembles, J. of AMS, 23:2 (2010), 535-561. 
[2] J. Anderson, Extensions, restrictions, and representations of states on $C^{*}$-algebras, Trans. Amer. Math. Soc., 249 (1979), 303-329.

[3] Artstein-Avidan, S., Giannopoulos, A. and Milman, V.D., Asymptotic geometric analysis, Part I, Vol. 202 (2015), American Mathematical Soc.

[4] R. F. Bass and K. Gröchenig, Random sampling of multivariate trigonometric polynomials, SIAM J. Math. Anal., 36(3) (2004/05), 773-795.

[5] J. Batson, D.A. Spielman, and N. Srivastava, Twice-Ramanujan Sparsifiers, SIAM Review, 56 (2014), 315-334.

[6] E.S. Belinsky, Interpolation and integral norms of hyperbolic polynomials, Mathematical Notes, 66 (1999), 16-23. Translation from Matem. Zametki, 66 (1999), 20-29.

[7] G. Bennett, V. Goodman, and C. Newman, Norms of random matrices, Pacific Journal of Mathematics, 59(2) (1975), 359-365.

[8] G. Bennett, L. E. Dor, V. Goodman, W. B. Johnson, and C. M. Newman, On uncomplemented subspaces of $L_{p}, 1<p<2$. Israel Journal of Mathematics, 26(2) (1977).

[9] S.N. Bernstein, Sur une classe de formules d'interpolation, Izv. AN SSSR, No. 9 (1931), 1151-1161.

[10] S.N. Bernstein, Sur une modification de la formule d'interpolation de Lagrange, Zapiski Khar'kovskogo matem. tovar., v. 5 (1932), 49-57.

[11] T. Bloom, L.P. Bos, J.-P. Calvi, N. Levenberg, Polynomial interpolation and approximation in $\mathbb{C}^{d}$, Ann. Polon. Math., 106 (2012), 53-81.

[12] A. Bondarenko, D. Radchenko, M. Viazovska, Optimal asymptotic bounds for spherical designs, Annales of Math., 178(2) (2013), 443452 .

[13] A. Bondarenko, D. Radchenko, M. Viazovska, Well-separated spherical designs, Constr. Approx., 41 (2015), no. 1, 93-112. 
[14] L. Bos and M. Vianello, Tchakaloff polynomial meshes, Ann. Polon. Math., 122 (2019), 221-231.

[15] J. Bourgain, Homogeneous polynomials on the ball and polynomial bases, Isr. J. Math., 68:2 (1989), 327-347.

[16] J. Bourgain, Bounded orthogonal systems and the $\Lambda(p)$-set problem, Acta Math., 162:1 (1989), 227-245.

[17] J. Bourgain, Random points in isotropic convex sets, Convex geometric analysis, 34 (1996), 53-58.

[18] J. Bourgain, On uniformly bounded bases in spaces of holomorphic functions, Amer. J. Math., 138:2 (2016), 571-584.

[19] J. Bourgain, J. Lindenstrauss, and V. Milman, Approximation of zonoids by zonotopes, Acta Math., 162 (1989), 73-141.

[20] J. Bourgain, A. Pajor, S.J. Szarek, N. Tomczak-Jaegermann, On the duality problem for entropy numbers of operators, In Geometric aspects of functional analysis, 1989, 50-63, Springer, Berlin, Heidelberg.

[21] J. Bourgain, L. Tzafriri, Invertibility of 'large' submatrices with applications to the geometry of Banach spaces and harmonic analysis, Israel journal of mathematics 57:2 (1987), 137-224.

[22] J. Bourgain, L. Tzafriri, Restricted invertibility of matrices and applications, Analysis at Urbana, Vol. II (Urbana, IL, 1986-1987), Vol. 138, Cambridge Univ. Press Cambridge, 1989, 61-107.

[23] J. Bourgain, L. Tzafriri, On a problem of Kadison and Singer, Journal für die reine und angewandte Mathematik (Crelles Journal), $\mathbf{4 2 0}$ (1991), 1-44.

[24] M. Bownik, Continuous frames and the Kadison-Singer problem, Coherent states and their applica tions,, Springer Proc. Phys., $20563-$ 88, Springer, Cham, 2018.

[25] S. Brazitikos, A. Giannopoulos, P. Valettas, and B.H. Vritsiou, Geometry of isotropic convex bodies, American Mathematical Soc., 196, 2014. 
[26] G. Brown, F. Dai, Approximation of smooth functions on compact two-point homogeneous spaces, J. Funct. Anal., 220 (2005), no. 2, 401-423.

[27] A. Cohen and G. Migliorati, Optimal weighted least-squares methods, SMAI J. Computational Mathematics, 3 (2017), 181-203.

[28] A. Cohen and M. Dolbeault, Optimal pointwise sampling for $L^{2}$ approximation, arXiv:2105.05545v2 [math.NA] 1 Jun 2021.

[29] D. Coppersmith, T.J. Rivlin, The growth of polynomials bounded at equally spaced points, SIAM Journal on Mathematical Analysis, 23:4 (1992), 970-983.

[30] F. Cucker and S. Smale, On the mathematical foundations of learning, Bulletin of AMS, 39 (2001), 1-49.

[31] F. Dai, Multivariate polynomial inequalities with respect to doubling weights and $A^{\infty}$ weights, J. Funct. Anal., 235 (2006), 137-170.

[32] F. Dai and A. Prymak, $L^{p}$-Bernstein inequalities on $C^{2}$-domains, arXiv:2010.06728v1 [math.CA] 13 Oct 2020.

[33] F. Dai, A. Prymak, V.N. Temlyakov, and S.Yu. Tikhonov, Integral norm discretization and related problems, Russian Math. Surveys 74:4 (2019), 579-630. Translation from Uspekhi Mat. Nauk 74:4(448) (2019), 3-58; arXiv:1807.01353v1.

[34] F. Dai, A. Prymak, A. Shadrin, V. Temlyakov, S. Tikhonov, Sampling discretization of integral norms, Constructive Approximation (2021), 1-17, arXiv:2001.09320v1 [math.CA] 25 Jan 2020.

[35] F. Dai, A. Prymak, A. Shadrin, V. Temlyakov, and S. Tikhonov, Entropy numbers and Marcinkiewicz-type discretization theorem, J. Functional Analysis, 281 (2021) 109090; arXiv:2001.10636v1 [math.CA] 28 Jan 2020.

[36] F. Dai, Feng and Heping Wang, Positive cubature formulas and Marcinkiewicz-Zygmund inequalities on spherical caps, Constr. Approx., 31 (2010), 1-36. 
[37] R. DeVore, G. Kerkyacharian, D. Picard, and V. Temlyakov, Mathematical methods for supervised learning, Found. Comput. Math., 6 (2006), 3-58.

[38] Z. Ditzian and A. Prymak, On Nikol'skii inequalities for domains in $\mathbb{R}^{d}$, Constr. Approx., 44 (2016), 23-51.

[39] Ding Dũng, V.N. Temlyakov, and T. Ullrich, Hyperbolic Cross Approximation, Advanced Courses in Mathematics CRM Barcelona, Birkhäuser, 2018; arXiv:1601.03978v2 [math.NA] 2 Dec 2016.

[40] Dinh Dũng and T. Ullrich, Lower bounds for the integration error for multivariate functions with mixed smoothness and optimal Fibonacci cubature for functions on the square, Math. Nachr., 288 (2014), 743762 .

[41] T. Figiel, J. Lindenstrauss, and V.D. Milman, The dimension of almost spherical sections of convex bodies, Acta Mathematica, 139:1 (1977), 53-94.

[42] F. Filbir and H.N. Mhaskar, Marcinkiewicz-Zygmund measures on manifolds, J. Complexity, 27 (2011), 568-596.

[43] S. Foucart and H. Rauhut, A Mathematical Introduction to Compressive Sensing, Springer, New York, 2013.

[44] A.A. Giannopoulos and V.D. Milman, Concentration property on probability spaces, Advances in Mathematics, 156:1 (2000), 77-106.

[45] E.D. Gluskin, Extremal properties of orthogonal parallelepipeds and their applications to the geometry of Banach spaces, Mathematics of the USSR-Sbornik, 64:1 (1989), 85-96.

[46] B. Gray, Homotopy theory, New York, San Francisco, London, Academic Press, 1975.

[47] P.G. Grigoriev, Estimates for Norms of Random Polynomials and Their Application, Mathematical Notes, 69:6 (2001), 868-872

[48] P.G. Grigoriev, Random Linear Combinations of Functions from $L_{1}$, Mathematical Notes, 74:2 (2003), 185-211 
[49] K. Gröchenig, Irregular sampling, Toeplitz matrices, and the approximation of entire functions of exponential type, Math. Comp., 68(226) (1999), 749-765.

[50] K. Gröchenig, Sampling, Marcinkiewicz-Zygmund inequalities, approximation, and quadrature rules, J. Approx. Theory, 257 (2020).

[51] K. Gröchenig, B.M. Pötscher, H. Rauhut, Learning Trigonometric Polynomials from Random Samples and Exponential Inequalities for Eigenvalues of Random Matrices, arXiv:math/0701781v2 [math.PR] 9 Nov 2010.

[52] O. Guedon and M. Rudelson, $L_{p}$-moments of random vectors via majorizing measures, Advances in Mathematics, 208:2 (2007), 798-823.

[53] O. Guédon, A.E. Litvak, A. Pajor, and N. Tomczak-Jaegermann, On the interval of fluctuation of the singular values of random matrices, J. European Math. Soc., 19:5 (2017), 1469-1505.

[54] L. Györfy, M. Kohler, A. Krzyzak, and H. Walk, A distribution-free theory of nonparametric regression, Springer, Berlin, 2002.

[55] R. van Handel, Chaining, interpolation and convexity II: The contraction principle, The Annals of Probability, 46 (2018), 1764-1805.

[56] N.J. Harvey and N. Olver, Pipage rounding, pessimistic estimators and matrix concentration, Proc. of the Twenty-Fifth Annual ACMSIAM Symposium on Discrete Algorithms (2014), 926-945.

[57] S. Heinrich, On the complexity of computing the $L_{q}$ norm, J. Complexity 49 (2018), 1-26.

[58] L. Hörmander, A Bernstein type inequality, unpublished manuscript, 1985.

[59] W.B. Johnson and G. Schechtman, Embedding $l_{p}^{m}$ into $l_{1}^{n}$, Acta Mathematica, 149:1 (1982), 71-85

[60] W.B. Johnson and G. Schechtman, Finite dimensional subspaces of $L_{p}$, Handbook of the geometry of Banach spaces, Vol. 1 (2001), 837870, North-Holland, Amsterdam. 
[61] R.V. Kadison and I.M. Singer, Extensions of pure states, Amer. J. Math., 81 (1959), 384-400.

[62] S. Kaczmarz, H. Steinhaus, Theorie der Orthogonalreihen, WarszawaLwow, 1935.

[63] N.J. Kalton and L. Tzafriri, The behaviour of Legendre and ultraspherical polynomials in $L_{p}$-spaces, Can. J. Math., 50 (1998), 12361252.

[64] R. Kannan, L. Lovasz, and M. Simonovits, Random walks and an $O *\left(n^{5}\right)$ volume algorithm for convex bodies, Random Structures and Algorithms, 11:1 (1997), 1-50.

[65] B.S. Kashin, Widths of certain finite-dimensional sets and classes of smooth functions, Izv. Akad. Nauk Armyan. SSR Ser. Mat., 41 (1977), 334-351. English transl. in Math. Izv, 11.

[66] B.S. Kashin, A property of bilinear forms (Russian), Soobshch. Akad. Nauk Gruzin. SSR, 97 (1980), no. 1, 29-32.

[67] B.S. Kashin, Some properties of matrices of bounded operators from the space $l_{2}^{n}$ into $l_{2}^{m}$, Izv. Akad. Nauk Armyan. SSR Ser. Mat., 15:5 (1980), $379-394$.

[68] B.S. Kashin, On homogeneous polynomials on the complex sphere, Matem. USSR Sbornik, 126 (1985), 420-425.

[69] B.S. Kashin, On trigonometric polynomials with coefficients in absolute value equal to either zero or one, Trudy 3rd Saratov winter school, 1 (1987), 19-30.

[70] B.S. Kashin, Lunin's method for selecting large submatrices with small norm, Sbornik Math., 206:7 (2015), 980-987.

[71] B.S. Kashin, Decomposing an orthogonal matrix into two submatrices with extremally small (2,1)-norm, Russian Math. Surveys, 72:5 (2017), 971-973.

[72] B.S. Kashin, S.V. Konyagin, and V.N. Temlyakov, Sampling discretization of the uniform norm, arXiv:2104.01229v2 [math.NA] 19 Jul 2021. 
[73] B.S. Kashin, I.V. Limonova, Decomposing a Matrix into two Submatrices with Extremally Small (2,1)-Norm, Math. Notes, 106:1 (2019), $63-70$.

[74] B.S. Kashin and V.N. Temlyakov, On the best $m$-term approximation and the entropy of sets in the space $L_{1}$, Matem. Zametki, 56 (1994), 59-86; English translation in Math. Notes, 56 (1994), No. 5-6, 11371157.

[75] B.S. Kashin and V.N. Temlyakov, On a norm and related applications, Matem. Zametki, 64 (1998), 637-640.

[76] B.S. Kashin and V.N. Temlyakov, On a norm and approximation characteristics of classes of functions of several variables, Metric theory of functions and related problems in analysis, Izd. Nauchno-Issled. Aktuarno-Finans. Tsentra (AFTs), Moscow, 1999, 69-99.

[77] B.S. Kashin and V.N. Temlyakov, The volume estimates and their applications, East J. Approx., 9 (2003), 469-485.

[78] B.S. Kashin and V.N. Temlyakov, Observations on discretization of trigonometric polynomials with given spectrum, Russian Math. Surveys, 73:6 (2018), 1128-1130. Translation from Uspekhi Mat. Nauk 73:6 (2018) 197-198.

[79] B. Kashin and L. Tzafriri, Some remarks on the restrictions of operators to coordinate subspaces, Preprint no. 12, Hebrew Univ. of Jerusalem, Jerusalem 1993/94, 14 pp., www.mi.ras.ru/ kashin/download/preprint93.pdf.

[80] B. Kashin and L. Tzafriri, Lower estimates for the supremum of some random processes II, East J. Approx., 3:1 (1995), 373-377.

[81] K.S. Kazarian, P.I. Lizorkin, Multipliers, bases, unconditional bases in the weighted spaces B and SB, Trudy MIAN im. Steklova, 187 (1989), 98-115; English translation in Proc. Steklov Inst. of Math., 187 (1990), 111-130.

[82] L. Kämmerer, T. Ullrich, and T. Volkmer, Worst-case recovery guarantees for least squares approximation using random samples, Constructive Approximation, 54 (2021), 295-352. 
[83] J. Keiner, S. Kunis, and D. Potts, Efficient Reconstruction of Functions on the Sphere from Scattered Data, J Fourier Anal. Appl., 13, (2007), 435-458. https://doi.org/10.1007/s00041-006-6915-y.

[84] S.V. Konyagin and V.N. Temlyakov, Some error estimates in Learning Theory, in the book Approximation Theory: A volume dedicated to Borislav Bojanov, Marin Drinov Acad. Publ. House, Sofia, 2004, 126144.

[85] S.V. Konyagin and V.N. Temlyakov, The Entropy in Learning Theory. Error Estimates, Constr. Approx., 25 (2007), 1-27.

[86] E. Kosov, Marcinkiewicz-type discretization of $L^{p}$-norms under the Nikolskii-type inequality assumption, J. Math. Anal. Appl., 504:1 (2021), 125358, doi:10.1016/j.jmaa.2021.125358

[87] E. Kosov, Remarks on sampling discretization, manuscript, 2021.

[88] D. Krieg and M. Ullrich, Function values are enough for $L_{2}$-approximation, Found. Comp. Math., doi:10.1007/s10208-020-09481-w; arXiv:1905.02516v4 [math.NA] 19 Mar 2020.

[89] D. Krieg and M. Ullrich, Function values are enough for $L_{2^{-}}$ approximation: Part II, J. Complexity, doi:10.1016/j.jco.2021.101569; arXiv:2011.01779v1 [math.NA] 3 Nov 2020.

[90] A. Kroó, On the existence of optimal meshes in every convex domain on the plane, J. Approx. Theory, 238 (2019), 26-37.

[91] S. Kullback and R.A. Leibler, On information and sufficiency, Annals of Mathematical Statistics, 22 (1) (1951), 79-86.

[92] M. Ledoux and M. Talagrand, Probability in Banach Spaces: isoperimetry and processes, Springer Science \& Business Media, 2013.

[93] D. Lewis, Finite dimensional subspaces of $L_{p}$, Studia Math, 63:2 (1978), 207-212.

[94] I.V. Limonova, Decomposing a matrix into two submatrices with smaller (2,1)-norms, Russian Math. Surveys, 71:4 (2016), 781-783. 
[95] I.V. Limonova, Decomposing a Matrix into Two Submatrices with Extremely Small Operator Norm, Math. Notes, 108:1 (2020), 137141.

[96] I. Limonova and V. Temlyakov, On sampling discretization in $L_{2}$, arXiv:2009.10789v1 [math.FA] 22 Sep 2020 (v2, 21 Apr 2021).

[97] A. A. Lunin, Operator norms of submatrices, Math. Notes, 45:3 (1989), 248-252. Translation from Mat. Zametki, 45:3 (1989) 94-100.

[98] J. Marcinkiewicz, Sur l'interpolation (I), Studia Mathematica 6 (1936), $1-17$.

[99] A. Marcus, D.A. Spielman, and N. Srivastava, Interlacing families II: Mixed characteristic polynomials and the Kadison-Singer problem, Annals of Math., 182:1 (2015), 327-350.

[100] G. Mastroianni and V. Totik, Weighted polynomial inequalities with doubling and $A^{\infty}$ weights, Constr. Approx., 16 (2000), no. 1, 37-71.

[101] H.N. Mhaskar, F.J. Narcowich, J.D. Ward, Spherical MarcinkiewiczZygmund inequalities and positive quadrature, Math. of Computation, 70 (2001), N 235, 1113-1130.

[102] S. Mendelson, A few notes on Statistical Learning Theory, In Advanced Lectures in Maching Learning, LNCS, 2600, Springer, 2003, $1-40$.

[103] S. Mendelson, Approximating $L_{p}$ unit balls via random sampling, Advances in Mathematics, 386 (2021), 107829.

[104] S. Mendelson and A. Pajor, On singular values of matrices with independent rows, Bernoulli, 12 (2006), 761-773.

[105] S. Mendelson and G. Paouris, On the singular values of random matrices, Journal of the European Mathematical Society, 16(4) (2014), $823-834$.

[106] S. Mendelson and G. Paouris, On generic chaining and the smallest singular value of random matrices with heavy tails, Journal of Functional Analysis, 262(9) (2012), 3775-3811. 
[107] M. Moeller and T. Ullrich, $L_{2}$-norm sampling discretization and recovery of functions from RKHS with finite trace, arXiv:2009.11940v2 [math.NA] 29 Apr 2021.

[108] H. L. Montgomery, Topics in Multiplicative Number Theory, Springer, 1971

[109] S. Nitzan, A. Olevskii, and A. Ulanovskii, Exponential frames on unbounded sets, Proc. Amer. Math. Soc., 144:1 (2016), 109-118.

[110] N. Nagel, M. Schäfer, T. Ullrich, A new upper bound for sampling numbers, Found. Comp. Math., Pub Date: 2021-04-26, DOI: 10.1007/s10208-021-09504-0; arXiv:2010.00327v1 [math.NA] $30 \mathrm{Sep}$ 2020.

[111] A. Naor and P. Youssef, Restricted invertibility revisited, A Journey Through Discrete Mathematics, Springer, 2017, 657-691.

[112] E. Novak, Deterministic and Stochastic Error Bounds in Numerical Analysis, Springer-Verlag, Berlin, 1988.

[113] E. Novak and H. Wozniakowski, Tractability of multivariate problems, Vol. 1: Linear information, volume 6 of EMS Tracts in Mathematics, European Mathematical Society (EMS), Zürich, 2008.

[114] E. Novak and H. Wozniakowski, Tractability of multivariate problems, Volume II: Standard information for functionals, volume 12 of EMS Tracts in Mathematics, European Mathematical Society (EMS), Zürich, 2010.

[115] E. Novak and H. Wozniakowski, Tractability of multivariate problems, Volume III: Standard information for operators, volume 18 of EMS Tracts in Mathematics. European Mathematcal Society (EMS), Zürich, 2012.

[116] A.M. Olevskii and A. Ulanovskii, Functions with Disconnected Spectrum: Sampling, Interpolation, Translates, Amer. Math. Soc., University Lecture Series, 65, Providence, Rhode Island, 2016.

[117] R. I. Oliveira, Sums of random Hermitian matrices and an inequality by Rudelson, Electr. Comm. Probab., 15 (2010), 203-212. 
[118] J. Ortega-Cerdá and J. Saludes, Marcinkiewicz-Zygmund inequalities J. Approx. Theory, 145(2) (2007), 237-252.

[119] P. Oswald, W. Zhou, Random reordering in SOR-type methods. Numerische Mathematik, 135:4 (2017), 1207-1220.

[120] G. Paouris, Concentration of mass on convex bodies, Geometric and Functional Analysis, 16:5 (2006), 1021-1049.

[121] S. Paul, M. Magdon-Ismail, P. Drineas, Feature selection for linear SVM with provable guarantees, Pattern Recognition, vol. 60 (2016), 205-214.

[122] I.Z. Pesenson, Sampling, splines and frames on compact manifolds, Intern. J. on Geomathematics, 6(1) (2015), 43-81.

[123] D.E. Pollard, A user's guide to measure theoretic probability, Cambridge University Press, 2002.

[124] D. Potts, G. Steidl, and M. Tasche, Fast Fourier transforms for nonequispaced data: a tutorial, In Modern sampling theory, Appl. Numer. Harmon. Anal., pp. 247-270, Birkhäuser Boston, Boston, MA, 2001.

[125] K. Pozharska and T. Ullrich, A note on sampling recovery of multivariate functions in the uniform norm, arXiv:2103.11124v2 [math.NA] 2 Apr 2021.

[126] A. Prymak, Geometric computation of Christoffel functions on planar convex domains, arXiv:12833v2 [math.CA] 29 Apr 2020.

[127] H. Rauhut, Compressive sensing and structured random matrices, In M. Fornasier, editor, Theoretical Foundations and Numerical Methods for Sparse Recovery, $\mathbf{9}$ of Radon Series on Computational and Applied Mathematics. de Gruyter, Berlin, 2010.

[128] M. Rudelson, Contact points of convex bodies, Israel J. Math., 101 (1997), 93-124.

[129] M. Rudelson, Almost orthogonal submatrices of an orthogonal matrix, Israel J. Math., 111 (1999), 143-155. 
[130] M. Rudelson, Random vectors in the isotropic position, Journal of Functional Analysis, 164:1 (1999), 60-72.

[131] G. Schechtman, Fine embeddings of finite-dimensional subspaces of $L_{p}, 1 \leq p<2$, into $l_{1}^{m}$, Proceedings of the American Mathematical Society, 94:4 (1985), 617-623.

[132] G. Schechtman, More on embedding subspaces of $L_{p}$ in $\ell_{r}^{n}$, Compositio Math., 61:2 (1987), 159-169.

[133] G. Schechtman, Tight embedding of subspaces of $L_{p}$ in $\ell_{p}^{n}$ for even $p$, Proc. AMS, 139 (2011), no. 12, 4419-4421.

[134] G. Schechtman, A. Zvavitch, Embedding Subspaces of $L_{p}$ into $\ell_{p}^{N}$, $0<p<1$, Mathematische Nachrichten, 227:1 (2001), 133-142.

[135] K. Seip, Interpolation and Sampling in Spaces of Analytic Functions, AMS University lectures series, 33, 2004.

[136] I.I. Sharapudinov, Estimating the $L_{p}$-norm of an algebraic polynomial in terms of its values at the nodes of a uniform grid, Sbornik: Mathematics, 188(12):1861 (1997).

[137] I.I. Sharapudinov, Polynomials orthogonal on grids, Makhachkala, 1997 (in Russian).

[138] M.A. Skopina, On the norms of polynomials in systems of periodic wavelets in the spaces $L_{p}$, Math. Notes, 59 (1996), N 5, 565-568.

[139] E. Slutsky, Alcuni proposizioni sulla teoria degli funzioni aleatovie, Giorn. Instituto Ital. degli Attuori, Anno VIII, No. 2, 1937.

[140] E. Slutsky, Selected papers, Academy of science of USSR, Moscow, 1960.

[141] S. Smale and D.-X. Zhou, Shannon sampling and function reconstruction from point values, Bull. Amer. Math. Soc. (N.S.), 41(3) (2004), 279-305.

[142] D.A. Spielman, Graphs, vectors, and matrices, Bulletin of the American Mathematical Society, 54:1, (2017) 45-61. 
[143] D.A. Spielman, N. Srivastava, An elementary proof of the restricted invertibility theorem, Isr. J. Math., 190, (2012), 83-91.

[144] N. Srivastava, On contact points of convex bodies, Geometric aspects of functional analysis, vol 2050, Springer, Berlin, Heidelberg, 2012, 393-412.

[145] N. Srivastava, Windows on theory: Discrepancy, graphs, and the Kadison-Singer problem, Asia Pac. Math. Newsl., 3 (2013) no.4, 1520 .

[146] N. Srivastava, Interlacing Families Open Problems, Manuscript, (2015), https://math.berkeley.edu/ nikhil/courses/270/open.pdf

[147] N. Srivastava and R. Vershynin, Covariance estimation for distributions with $2+\varepsilon$ moments, Annals Prob., 41:5 (2013), 3081-3111.

[148] M. Talagrand, Upper and lower bounds for stochastic processes: modern methods and classical problems, Springer Science and Business Media, 2014.

[149] M. Talagrand, Embedding subspaces of $L_{1}$ into $\ell_{1}^{N}$, Proceedings of AMS, 108:2 (1990), 363-369.

[150] M. Talagrand, Embedding subspaces of $L_{p}$ in $\ell_{p}^{N}$, Geometric Aspects of Functional Analysis: Israel Seminar (GAFA), 1992-94. Vol. 77. Birkhäuser, 2012.

[151] V.N. Temlyakov, Approximation of periodic functions of several variables with bounded mixed derivative, Trudy MIAN, 156 (1980), 233260; English transl. in Proceedings of Steklov Inst. Math., 2 (1983).

[152] V.N. Temlyakov, On Approximate Recovery of Functions with Bounded Mixed Derivative, J. Complexity, 9 (1993), 41-59.

[153] V.N. Temlyakov, Greedy Approximation, Cambridge University Press, 2011.

[154] V.N. Temlyakov, The Marcinkiewicz-type discretization theorems for the hyperbolic cross polynomials, Jaen Journal on Approximation, $\mathbf{9}$ (2017), No. 1, 37-63; arXiv:1702.01617v2 [math.NA] 26 May 2017. 
[155] V.N. Temlyakov, The Marcinkiewicz-type discretization theorems, Constructive Approximation, 48 (2018), 337-369; arXiv:1703.03743v1 [math.NA] 10 Mar 2017.

[156] V.N. Temlyakov, Universal discretization, Journal of Complexity, 47 (2018), 97-109; arXiv:1708.08544v1 [math.NA] 28 Aug 2017.

[157] V. Temlyakov, Multivariate Approximation, Cambridge University Press, 2018.

[158] V. Temlyakov, Sampling discretization error for integral norms for function classes, J. Complexity, 54 (2019), 101408; arXiv:1812.08100v1 [math.NA] 19 Dec 2018.

[159] V. Temlyakov, Sampling discretization of integral norms of the hyperbolic cross polynomials, Trudy MIAN, 312 (2021), 282-293; Engl. Translation in Proceedings of the Steklov Institute of Mathematics, 312 (2021), 270-281; arXiv:2005.05967v1 [math.NA] 12 May 2020.

[160] V. Temlyakov, A remark on entropy numbers, arXiv:2008.13030v1 [math.NA] 29 Aug 2020.

[161] V.N. Temlyakov, On optimal recovery in $L_{2}$, J. Complexity 65 (2021), 101545; arXiv:2010.03103v1 [math.NA] 7 Oct 2020.

[162] V.N. Temlyakov and T. Ullrich, Bounds on Kolmogorov widths of classes with small mixed smoothness, J. Complexity, Available online 4 May 2021, 101575; arXiv:2012.09925v1 [math.NA] 17 Dec 2020.

[163] K. Tikhomirov, Sample covariance matrices of heavy-tailed distributions. IMRN, 2018:20 (2018), 6254-6289.

[164] J.F. Traub, G.W. Wasilkowski, and H. Woźniakowski, InformationBased Complexity, Academic Press, Inc., 1988.

[165] J.A. Tropp, User-friendly tail bounds for sums of random matrices, Foundations of Comput. Math., 12 (2012), 389-434.

[166] V. Vapnik, Statistical Learning Theory, John Wiley\& Sons, Inc., New York, 1998. 
[167] S. Van de Geer, Empirical Process in M-Estimaton, Cambridge University Press, New-York, 2000.

[168] R. Vershinin, John's decompositions: selecting a large part, Israel $J$. Math., 122 (2001), 253-277.

[169] R. Vershynin, Approximating the moments of marginals of highdimensional distributions, The Annals of Probability, 39:4 (2011), 1591-1606.

[170] R. Vershynin, How close is the sample covariance matrix to the actual covariance matrix?, J. Theor. Prob., 25:3 (2012), 655-686.

[171] G.W. Wasilkowski, Some nonlinear problems are as easy as the approximation problem, Comput. Math. with Appls., 10 (1984), 351363.

[172] G.W. Wasilkowski and H. Woźniakowski, On the Power of Standard Information for Weighted Approximation, Found. Comput. Math. (2001) 1:417-434.

[173] P. Wojtaszczyk, On values of homogeneous polynomials in discrete sets of points, Studia Math., 84 (1986), 97-104.

[174] H. Woźniakowski, A survey of information-based complexity, J. Complexity, 1 (1985), 11-44.

[175] A. Zvavitch, More on embedding subspaces of $L_{p}$ into $\ell_{p}^{N}, 0<p<1$, Geometric Aspects of Functional Analysis, Springer, Berlin, Heidelberg, 2000, 269-280.

[176] A. Zygmund, Trigonometric Series, Cambridge University Press, 1959. 